\title{
NANO-MECHANICS OF CARTILAGE GLYCOSAMINOGLYCANS USING MOLECULAR DYNAMICS METHODS
}

\author{
A Thesis \\ presented to \\ the Faculty of California Polytechnic State University, \\ San Luis Obispo
}

\author{
In Partial Fulfillment \\ of the Requirements for the Degree \\ Master of Science in Mechanical Engineering \\ by \\ Kevin Neil Hendrickson \\ December 2008
}


(C) 2008

Kevin Neil Hendrickson

ALL RIGHTS RESERVED 


\section{COMMITTEE MEMBERSHIP}

TITLE:

AUTHOR:

DATE SUBMITTED:

COMMITTEE CHAIR: $\quad$ Stephen Klisch, Ph.D.
Nano-mechanics of cartilage glycosaminoglycans using molecular dynamics methods

Kevin Neil Hendrickson

December 2008

COMMITTEE MEMBER: Andrew Davol, Ph.D.

COMMITTEE MEMBER: Thomas Mackin, Ph.D. 


\begin{abstract}
Nano-mechanics of cartilage glycosaminoglycans using molecular dynamics methods
\end{abstract}

Kevin Neil Hendrickson

Articular Cartilage (AC) is the main load carrying material in synovial joints \{Hamerman, 1962\} and degeneration of AC can cause pain in the form of arthritis. Current work is centered on the method of replacing damaged cartilage inside the body (in vivo) with tissue engineered outside the body (ex vivo) $\{$ Temenoff, 2000\}. In order to engineer tissue ex vivo similar to the native tissue in structure and function there must be a comprehensive understanding of the mechanical properties of AC. This work focuses on the study of glycosaminoglycans (GAGs), a molecule known to be primarily responsible for the compressive stiffness of $\mathrm{AC}$, using molecular dynamics methods. First, a single chain simulation is run to establish a chain length to use for the rest of the study. Then two more simulations are run that mimic a possible physical scenario for changing GAG density. The first is a five chain simulation that mimics the situation where GAG chains are compressed and pushed together. Pressure and density relations are generated and compared to the micro-structural level Donnan model \{Maroudas, 1979\} and Poisson-Boltzmann unit cell (PB) model \{Marcus, 1955\}. The last simulation imitates the scenario of one GAG chain sliding between two adjacent GAG chains. The work to pull the central chain through the adjacent chains is calculated and plotted at different chain spacing. A 20 disaccharide-unit long chain is found to be the most stable chain length, but for the purpose of saving computational time without a large loss in stability a 10 unit chain is used for the rest of the simulations. The pressure-density relations found from the five chain simulation are of the same magnitude as the micro-structural level models. Observations made based on the graphical playback of the pulling simulation give insight into the importance of ion interaction with the GAG chains. It was found to take more work to pull the chain with more open space around because of the binding nature of the ions coming between the chains. The tighter spaced chains allowed fewer ions to fit between chains creating less binding force, therefore taking less work to pull. This work can be scaled up to the next level using coarse-graining methods which will be more comparable to experimental work, possibly leading to results that will help characterize AC for better implementation of engineered tissue. 


\section{ACKNOWLEDGMENTS}

A special thanks to the Italian researchers of Politecnico di Milano for their generous hospitality, kindness, and wisdom.

Computaional Biomechanics Lab

Politecnico di Milano, Milano, Italy

Alberto Redaelli

Simone Vesentini

Marco Deriu 


\section{Table of Contents}

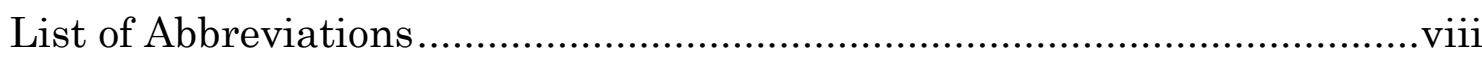

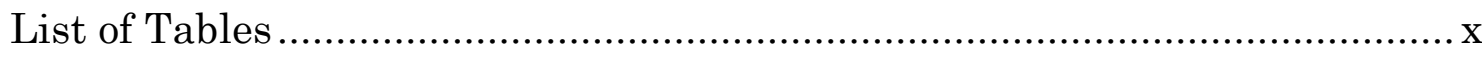

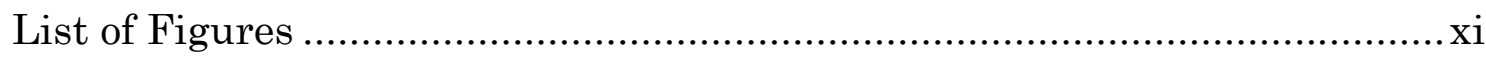

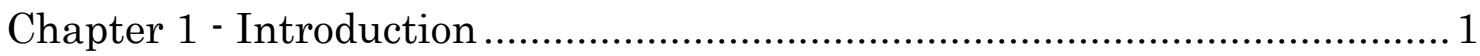

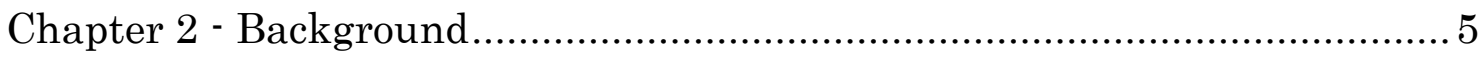

2.1 Articular Cartilage …................................................................ 5

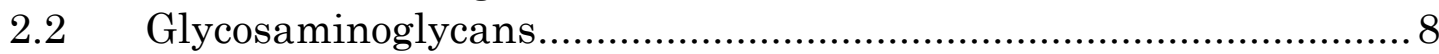

2.3 Biomechanical Properties ............................................................. 10

Chapter 3 - Molecular Dynamics - An Introduction ................................... 12

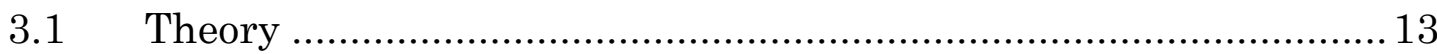

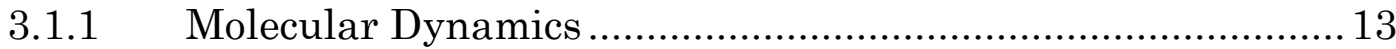

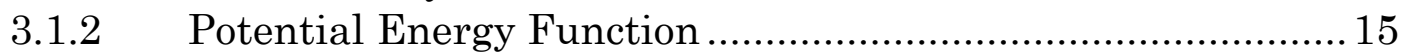

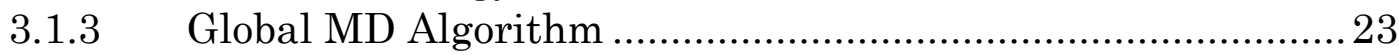

3.1.4 Treatment of non-bonded energy terms ……........................25

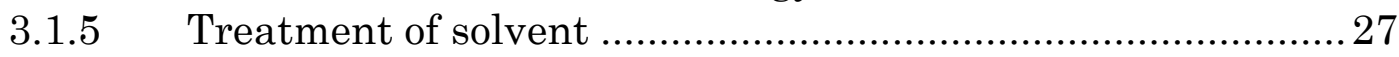

3.1.6 Periodic Boundary Conditions …….....................................28 
Chapter 4 - Methods

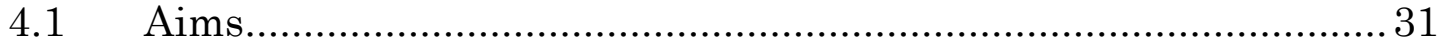

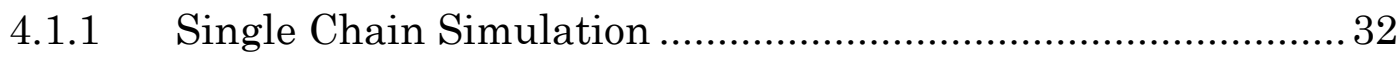

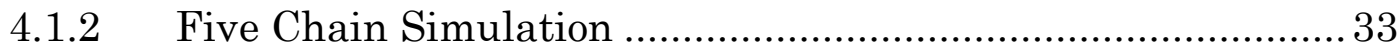

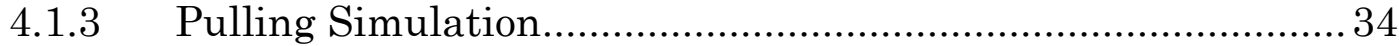

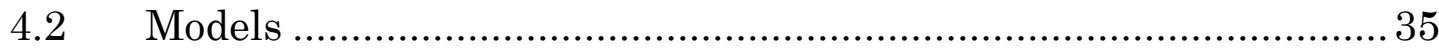

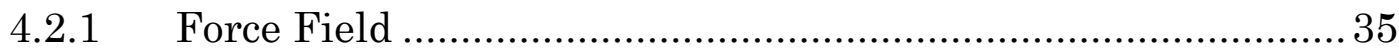

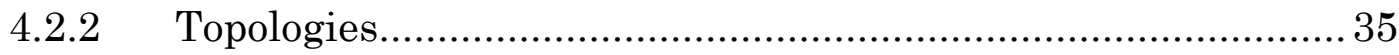

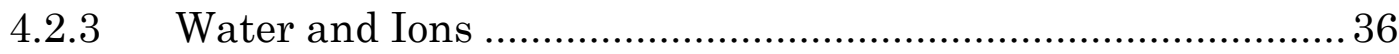

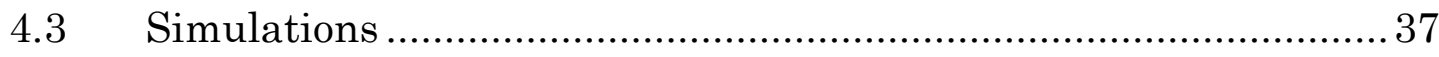

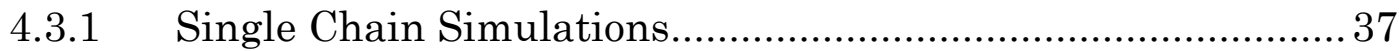

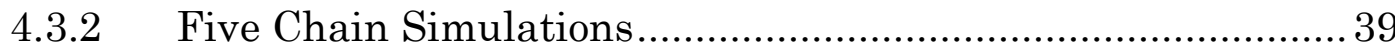

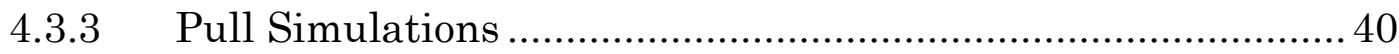

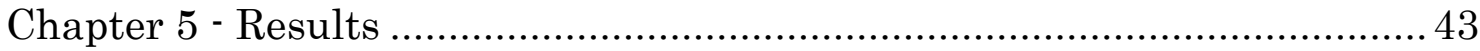

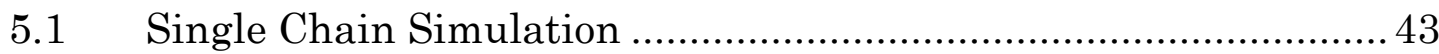

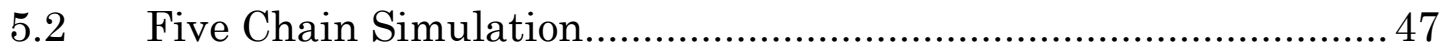

5.3 Pulling Simulation.................................................................. 49

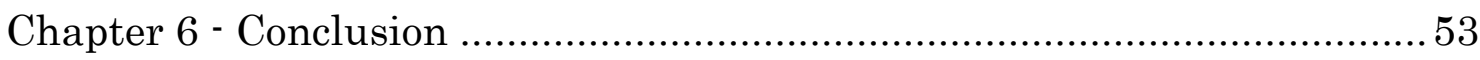

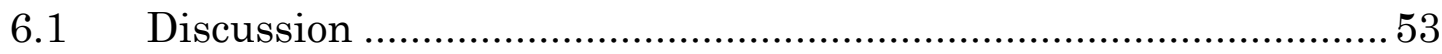

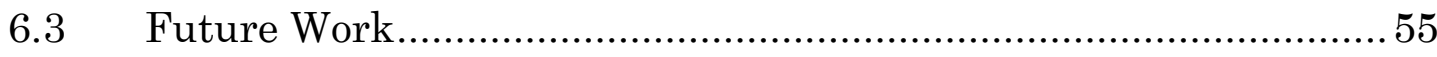

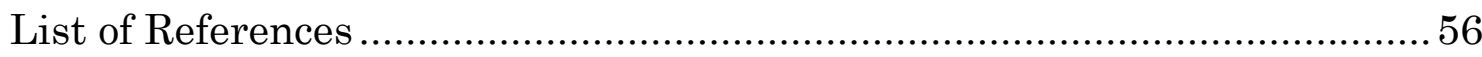

Appendix A - Derivation of Ionic Concentration...........................................59

Appendix B - Sample simulation times.....................................662

Appendix C - Steps to run a molecular dynamics simulation ..................63

Appendix D - Sample files..................................................66 


\section{List of Abbreviations}

$\begin{array}{ll}\AA & \text { Angstrom } \\ \text { AC } & \text { Articular Cartilage } \\ \text { C4S } & \text { Chondroitin-4-Sulfate } \\ \text { C6S } & \text { Chondroitin-6-Sulfate } \\ \text { CGM } & \text { Cartilage Growth Model } \\ \text { CH } & \text { Chondroitin } \\ \text { CHARMM } & \text { Chemistry at HARvard Macromolecular Mechanics } \\ \text { COL } & \text { Collagen } \\ \text { CS } & \text { Chondroitin Sulfate } \\ \text { Da } & \text { Dalton (atomic mass unit) } \\ \text { ECM } & \text { Extra-Cellular Matrix } \\ \text { G1 } & \text { Globular Domain 1 } \\ \text { G2 } & \text { Globular Domain 2 } \\ \text { G3 } & \text { Globular Domain 3 } \\ \text { GAG } & \text { Glycosaminoglycan } \\ \text { Gal } & \text { Galactose } \\ \text { GalNAc } & \text { N-Acetyl-D-Galactoamine } \\ \text { GalNAc4S } & \text { N-Acetyl-D-Galactoamine-6-Sulfate } \\ \text { GalNAc6S } & \text { N-Acetyl-D-Galactoamine-4-Sulfate } \\ & \end{array}$




$\begin{array}{ll}\text { GlcUA } & \text { D-Glucuronate } \\ \text { HA } & \text { Hyaluronan } \\ \text { KS } & \text { Keratin Sulfate } \\ \text { MD } & \text { Molecular Dynamics } \\ \mathrm{nm} & \text { Nanometer } \\ \mathrm{PB} & \text { Poisson-Boltzmann Unit Cell } \\ \mathrm{PG} & \text { Proteoglycan }\end{array}$




\section{List of Tables}

5.1 Average pressure data for 5 different GAG densities. ......................... 48

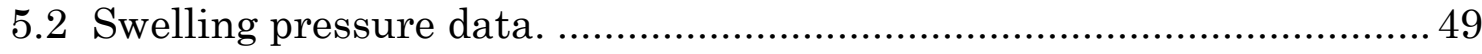

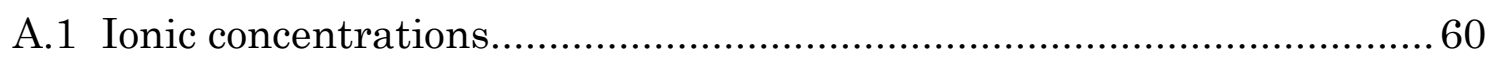




\section{List of Figures}

2.1: Diagram showing the composite like nature of $\mathrm{AC}$............................. 6

2.2: One molecule of aggrecan bound to HA ……....................................... 7

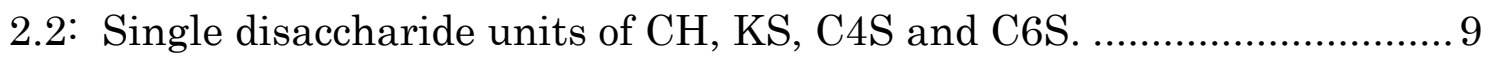

2.3: GAG chain with 4 C6S disaccharide units............................................. 9

2.4: GAG spacing and length along aggrecan core protein. ....................... 10

3.1: Diagram of bond pairs....................................................................... 17

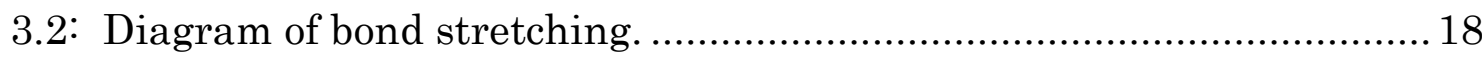

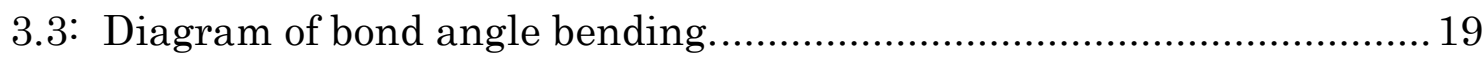

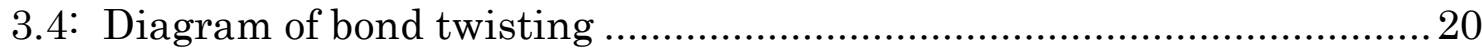

3.5: Lennard Jones 12-6 potential curve ..................................................22

3.6: Three different methods for defining the cutoff region ........................26

3.7: Two-dimensional Periodic Boundary Condition. ...................................29

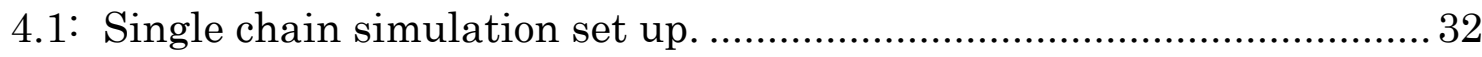

4.2: Physical relevance of the five chain simulations................................. 33 
4.3: Physical relavance of pulling simulation .............................................. 34

4.4: Set-up for the single chain simulations. ............................................... 38

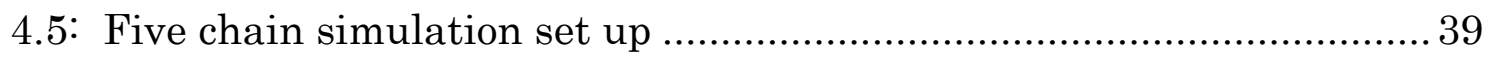

4.6: Schematic of pull simulation set up ................................................ 41

5.1: Figure showing how end-to-end length is calculated ..........................44

5.2: Single chain GAG end-to-end distance motion .................................... 45

5.3: Standard deviation of the normalized distance.................................. 46

5.4: Pressure vs. density compared of 5-chain simulation .......................... 48

5.5: Snapshot of pull simulation at $1.5 \mathrm{~nm}$ spacing.................................51

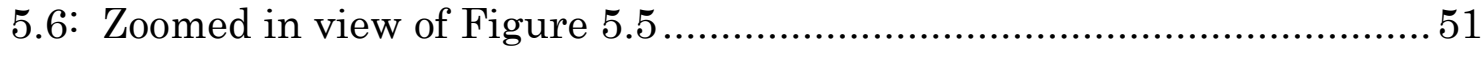

5.7: Plot of the work required to pull the central chain .............................52 


\section{Chapter 1}

\section{Introduction}

Articular Cartilage (AC) is the main load carrying material in synovial joints \{Hamerman, 1962\}. It also lubricates the joint and resists wear to provide a smooth sliding action between bones AAteshian, 1998\}. AC is subjected to intermittent contact pressures reaching $3-18 \mathrm{MPa}$ as many as a million times per year $\{$ Hodge, 1986\}. The extra-cellular matrix (ECM) of AC is composed of water (60-90\%) and a solid matrix consisting mostly of a cross-linked collagen (COL) network and proteoglycans (PGs), a fixed charge polymer. AC behaves like a fiber-reinforced composite material, where the "fibers" correspond to the COL network and the "matrix" is made up of PGs. Because it behaves like a fiber reinforced composite material it will have different mechanical properties in tension and compression. 
One major problem with $\mathrm{AC}$ is its avascularity, which prevents it from healing \{Buckwalter, 1998\}. Degeneration of the cartilage in joints can often lead to pain and discomfort in the form of arthritis \{Sandell, 2001\}. Arthritis is a common disability in the United States; $21 \%$ of adults are affected with some form of arthritis \{Lethbridge, 2006\}, however the medical techniques currently in place are inadequate for repairing or replacing damaged cartilage \{Smith, 2005\}. Current work is centered on the method of replacing damaged cartilage inside the body (in vivo) with tissue engineered outside the body (ex vivo) \{Temenoff, 2000\}.

In order to engineer tissue $e x$ vivo similar to the native tissue in structure and function there must be a comprehensive understanding of the mechanical properties of AC. The molecule in AC most responsible for the mechanical stiffness in compression is glycosaminoglycan (GAG). GAGs are long un-branched polysaccharides consisting of repeating units of disaccharides. GAGs resist compression due to the repulsion of its fixed negative charges. GAG chains are on the order of 20 to $60 \mathrm{~nm}$ in length in AC $\{$ Bathe, 2005\}, which makes them extremely difficult to analyze experimentally. Molecular dynamics methods have been used to analyze molecular behavior and gain insight into the relationship between the molecular structure, movement and function. With the increasing power and speed of computers, molecular dynamics has become a viable option for analyzing molecules. 
The scope of this project is to model the C6S GAG in several configurations that may be related to changing GAG density. This will lead the way to using coarse graining methods, which will be discuss in more detail in chapter 6, to eventually predict pressure-density relations. However, pressure-density calculations will be made on these models in order to evaluate how nano-level models compare with data for GAGs in AC tissue. Three specific aims were considered to mimic physiological scenarios of GAG molecules in compression.

1. An experiment with single GAG chains will be studied in order to choose a length or number of $\mathrm{C} 6 \mathrm{~S}$ disaccharide units to use in the molecular dynamics simulations. This is important because the size of the system should be short enough to minimize the use of computing resources but long enough to avoid boundary conditions like end effects and chain curvature which will effect the results.

2. A simulation will be investigated to compare density and pressure calculations of a nano-level GAG system to that of AC cartilage. Molecular dynamics simulations will be performed on five GAG chains at different spacing in order to calculate the pressure in the system. This model represents one mechanism of changing GAG density. These results will be compared to the Donnan model \{Maroudas, 1979\} and Poisson- 
Boltzmann unit cell (PB) model \{Marcus, 1955\} of AC GAG pressure and density.

3. The last experiment is modeled after another mechanism for changing GAG density where one GAG chain is pulled between two adjacent parallel chains. The work to pull the GAG chain will be calculated and compared among four simulations at different GAG spacing. 


\section{Chapter 2}

\section{Background}

\subsection{Articular Cartilage}

Cartilage is made up of two main components, a cross-linked network of collagen (50-60 percent of tissue dry weight) and the large aggregating proteoglycan, aggrecan (30-35 percent of tissue dry weight) \{Heinegard, 1989; Upholt, 1991\}. These two constituents of the solid matrix work together as a fiber-reinforced composite which give the cartilage special characteristics. In general, the collagen network resists tensile and shear stresses while the proteoglycans resist fluid flow and compressive stresses $\{$ Mow, 1988\}. There are no blood vessels in mature cartilage so it is fed through diffusion, propagated by the pumping action generated from the compression of the cartilage. 
Collagen is a long fibrous protein. It provides most of the tensile strength and elasticity of cartilage. The molecule wraps itself up into organized bundles called fibrils and groups of fibrils make collagen fibers.

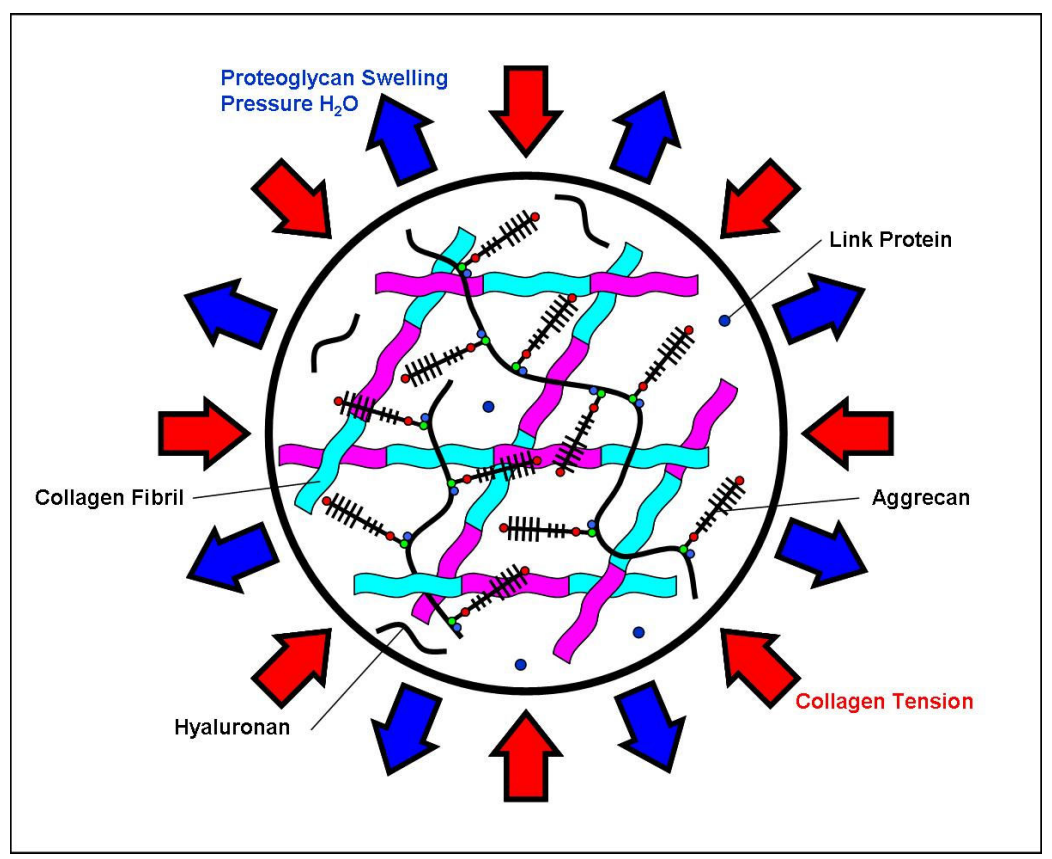

Figure 2.1: Diagram showing the composite like nature of AC. The tension in the collagen (red arrows) resists the swelling pressure provided by the proteoglycans (blue arrows).

Aggrecan is the predominant proteoglycan in articular cartilage. It is a modular proteoglycan with multiple functional domains: G1, G2, KS, CS and G3 which can be seen in Figure 2.2 \{Roughley, 2006\}. The globular domains G1, G2 and G3 are not of interest in this study because they are not known to contribute to the compressive properties of AC. However, the GAG region containing the keratin sulfate (KS) and chondroitin sulfate (CS) domains are of interest. A more detailed explanation of GAG's is provided in section 2.2. 


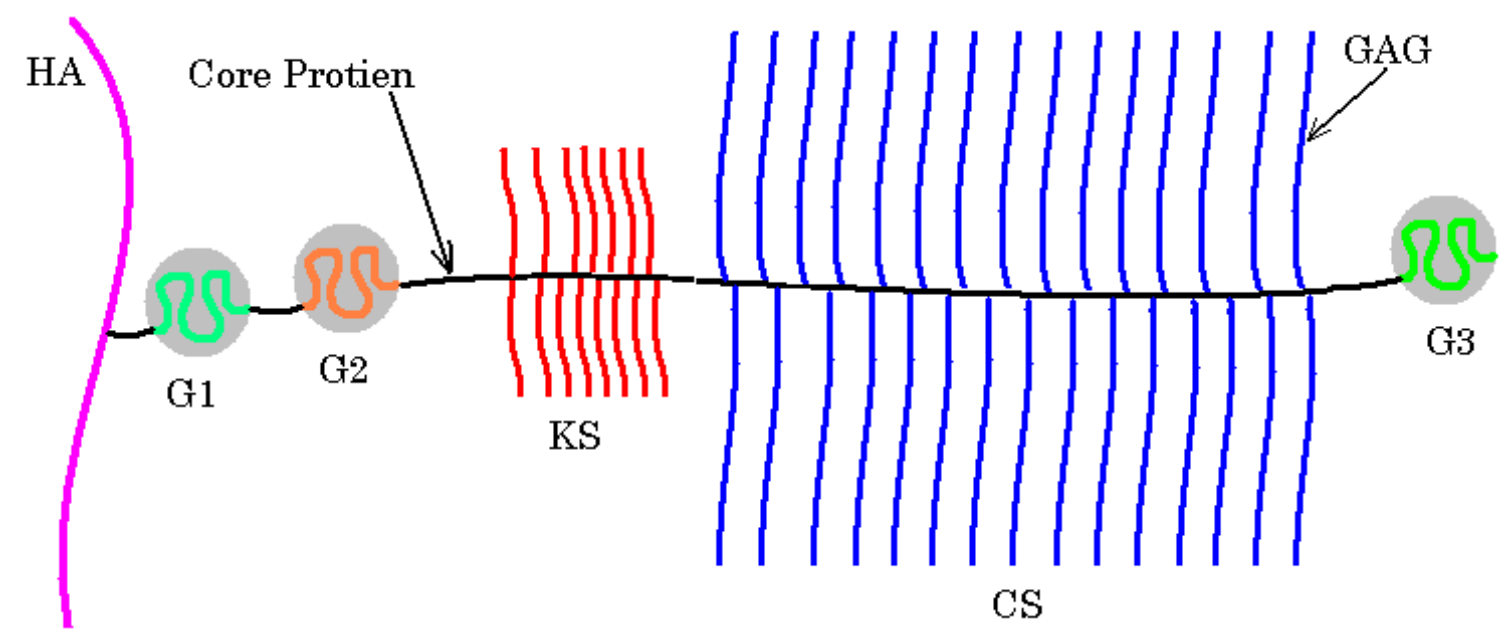

Figure 2.2: One molecule of aggrecan bound to HA. Many molecules of aggrecan bound to HA produces a PG aggregate. Aggrecan structure: G1, G2, KS, CS, G3 and Core Protein.

Aggrecan is often aggregated into high molecular weight supra-molecule structures called PG aggregates. Aggregation is accomplished by the interaction of aggrecan with hyaluronan (HA) and a link-protein \{Tsiganos, 1972\}. The molecular weight of a single molecule of aggrecan in the tissue ranges from 1 to $3.5 \mathrm{MDa}$ and the aggregates formed are of a molecular size up to $800 \mathrm{MDa}$ \{Heinegard, 1987\}. The molecular weight of a single GAG molecule is $10-30 \mathrm{kDa}$ corresponding to 20-60 disaccharide units with a contour length of 200-600 $\AA(20-60 \mathrm{~nm})$ \{Bathe, 2005\}. The contour length is defined as the length at maximum physically possible extension for a linear polymer chain \{IUPAC, 1997\}. 


\section{$2.2 \quad$ Glycosaminoglycans}

There are a few different forms of GAGs found in AC; chondroitin $(\mathrm{CH})$, keratin sulfate (KS), chondroitin-4-sulfate ( $\mathrm{C} 4 \mathrm{~S})$, and chondroitin-6-sulfate (C6S) are the most common. GAGs are produced from the assembly of repeating disaccharide units seen in Figure 2.3. The negatively charged groups $\mathrm{COO}^{-}$and $\mathrm{OSO}_{3}^{-}$generate a high density of fixed negative charge. Large repulsive forces are created when GAGs are forced together due to compression of the negative charge groups. These negative charge groups are what cause the resistance to compression that we are interested in. Both C4S and C6S have these negative charge groups, but there are higher concentrations of C6S in AC and almost no C4S in adult AC \{Mourao, 1988\}. For these reasons only $\mathrm{C} 6 \mathrm{~S}$ will be investigated in this study. 


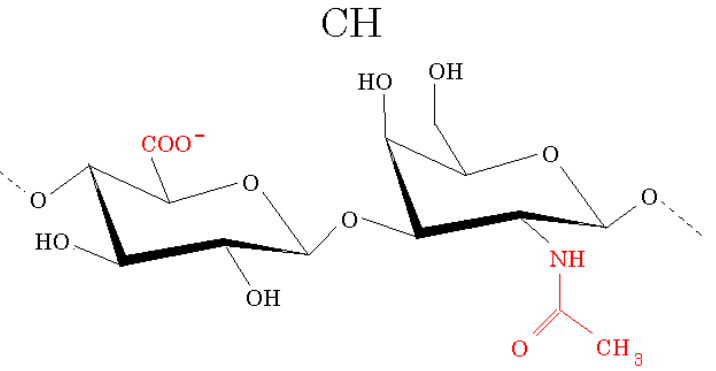

GlcUA

GalNAc
C4S

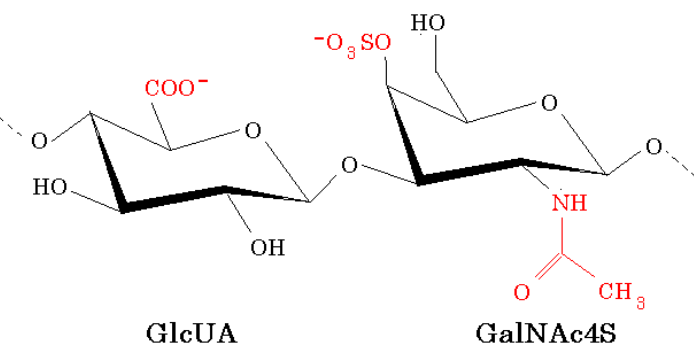

KS

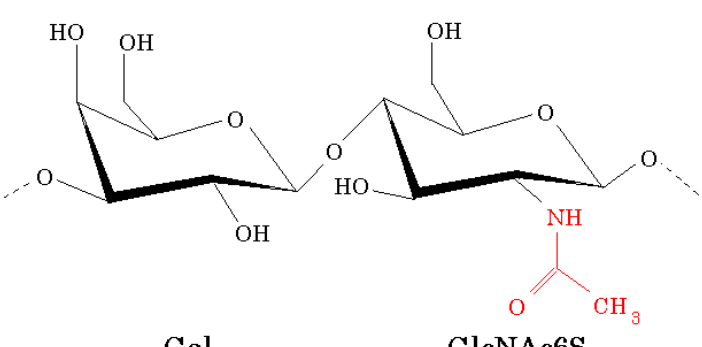

Gal

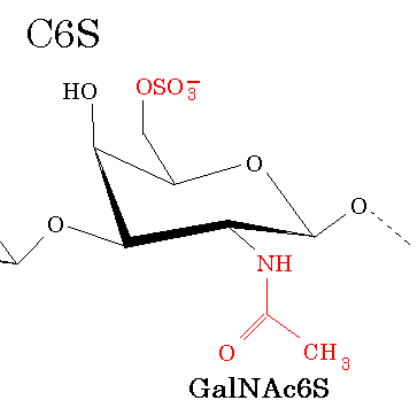

Figure 2.3: Single disaccharide units of $\mathrm{CH}, \mathrm{KS}, \mathrm{C} 4 \mathrm{~S}$ and C6S. Note the only physical difference between $\mathrm{C} 4 \mathrm{~S}$ and $\mathrm{C} 6 \mathrm{~S}$ is the location of the $\mathrm{OSO}_{3}{ }^{-}$group.

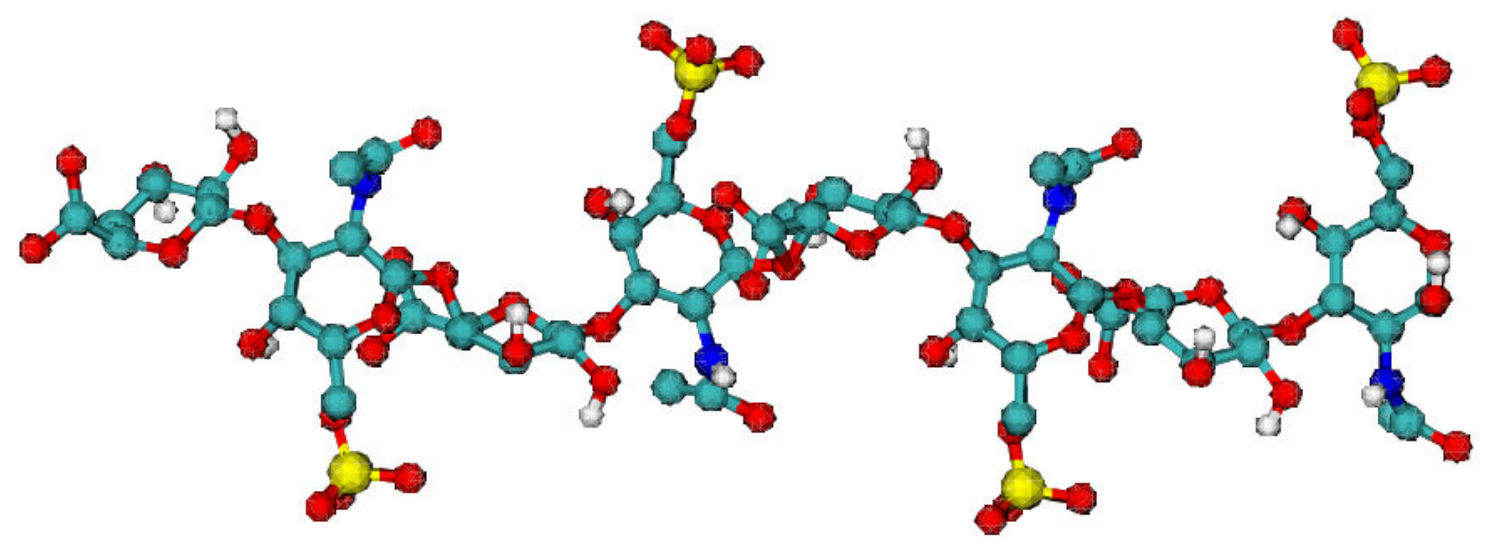

Figure 2.4: GAG chain with 4 C6S disaccharide units. Light blue = Carbon; Dark Blue $=$ Nitrogen $;$ Red $=$ Oxygen $;$ Yellow $=$ Sulfur; White $=$ Hydrogen 


\subsection{Biomechanical Properties}

The physiological spacing of the GAG chains along the core protein is important because it determines how the model will be set up. Data gathered using atomic force microscopy on bovine nasal cartilage shows GAG spacing in fetal aggrecan is $3.2+/-0.8 \mathrm{~nm}$ and mature is $4.4+/-1.2 \mathrm{~nm}\{\mathrm{Ng}, 2003\}$. Another important factor is the contour length or end-to-end length. Experimental studies show that the contour length of C6S is $20-60 \mathrm{~nm}\{\mathrm{Ng}$, 2003; Bathe, 2005\}

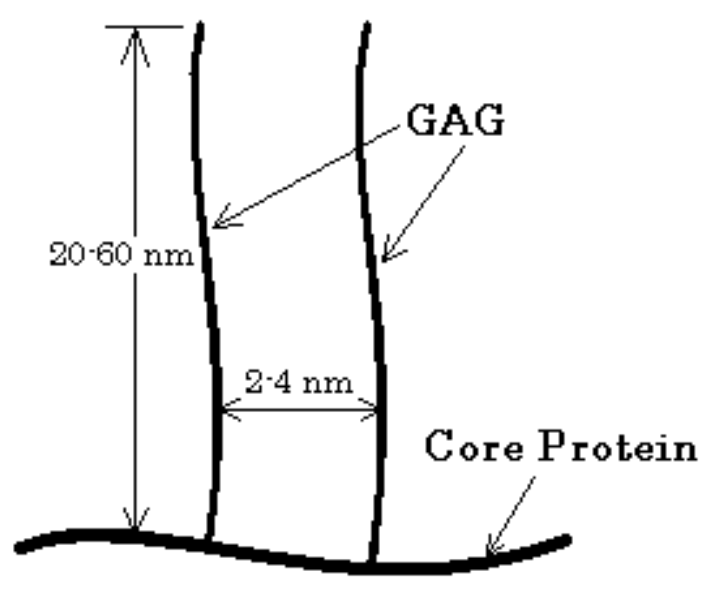

Figure 2.5: GAG spacing and length along aggrecan core protein.

Although GAG length is important another length property, called persistence length, is arguably more significant. The persistence length is defined as the length over which correlations in the direction of the tangent are lost. In other words a chain shorter than the persistence length will 
behave much like a highly flexible elastic rod and the behavior of a chain much longer than the persistence length can only be described statistically like a random 3-dimensional path. The persistence length of GAG chains was found to be $21 \mathrm{~nm}$ for fetal and $14 \mathrm{~nm}$ for mature aggrecan. With a $95 \%$ confidence interval this becomes $17-25 \mathrm{~nm}$ and $10-21 \mathrm{~nm}$, respectively $\{\mathrm{Ng}$, $2003\}$.

Typical PG concentrations in AC are 20-80 mg/ml \{Buschmann, 1995\}. Molecular Weight of one C6S unit is 442 Daltons (Da), which is related to $\mathrm{kg}$ via the conversion factor in Equation 2.1.

$$
1 D a=1.6605 \times 10^{-27} \mathrm{~kg}
$$




\section{Chapter 3}

\section{Molecular Dynamics - An Introduction}

The intent of this chapter is to educate those readers unfamiliar with molecular dynamics (MD) simulations. It is assumed that the reader has an understanding of classical mechanics, statistical methods and thermodynamics, but knows very little—if anything at all—on methods for atomistic computer simulations.

With the rapid increase in the speed of today's computers the computer experiment, or simulation, has inserted itself between experiment and theory as one of the chief methods of determining solutions. A model is still provided by theorists, but the calculations are carried out by the computer according to a given algorithm. 


\subsection{Theory}

\subsubsection{Molecular Dynamics}

Molecular dynamics is a computer simulation technique where the time evolution of a set of interacting atoms is followed by integrating their equations of motion \{Ercolessi, 1997\}. MD follows the laws of classical mechanics, most notably Newton's law:

$$
\mathbf{F}_{i}=m_{i} \mathbf{a}_{i}
$$

for each atom $i$ in a system constituted by $N$ atoms. Here, $m_{i}$ is the atom mass, $\mathbf{a}_{i}=d^{2} \mathbf{r}_{i} / d t^{2}$ is its acceleration, and $\mathbf{F}_{i}$ is the force acting upon it due to the interactions with other atoms. Given an initial set of positions and velocities the computer calculates a trajectory in a $6 \mathrm{~N}$-dimensional space $(3 \mathrm{~N}$ positions and $3 \mathrm{~N}$ momenta). However, such a trajectory is usually not very useful by itself. MD is a statistical mechanics method; it is a way to obtain a set of configurations distributed according to some statistical ensemble. A configuration is defined as one set of 3-dimensional position vectors for each atom at one moment in time. An ensemble is a collection of all possible configurations which have different microscopic states but have an identical 
macroscopic or thermodynamic state. Different definitions of the thermodynamic state lead to different classifications for ensembles:

- Microcanonical Ensemble (NVE): The thermodynamic state characterized by a fixed number of atoms, $N$, a fixed volume, $V$ and a fixed Energy, $E$.

- Canonical Ensemble (NVT): The thermodynamic state characterized by a fixed number of atoms, $N$, a fixed volume, $V$, and affixed temperature, $T$.

- Isobaric-Isothermal Ensemble (NPT): The thermodynamic state characterized by a fixed number of atoms, $N$, a fixed pressure, $P$, and fixed temperature, $T$.

- Grand Canonical Ensemble $(\mu V T)$ : The thermodynamic state characterized by a fixed chemical potential, $\mu$, a fixed volume, $V$, and fixed temperature, $T$.

William Gibbs defined chemical potential by stating, "If to any homogeneous mass in a state of hydrostatic stress we suppose an infinitesimal quantity of any substance to be added, the mass remaining homogeneous and its entropy and volume remaining unchanged, the increase of the energy of the mass divided by the quantity of the substance added is the potential for that substance in the mass considered." \{Gibbs, 1906\} 


\subsubsection{Potential Energy Function}

In order to model the physical system a function for the potential must be chosen. That is, a function $\Phi\left(\mathrm{r}_{1}, \ldots, \mathrm{r}_{N}\right)$ of the positions of the nuclei, representing the potential energy of the system when the atoms are arranged in that particular configuration. This function is usually constructed based on relative positions of the atoms with respect to each other, rather than absolute positions.

Forces are derived from the gradients of the potential function with respect to atomic displacements:

$$
\mathbf{F}_{i}=-\nabla_{\mathbf{r}_{i}} \Phi\left(\mathbf{r}_{1}, \ldots, \mathbf{r}_{N}\right)
$$

This form implies that the total energy, $E=K+\Phi$, is conserved, where $K$ is the instantaneous kinetic energy.

The simplest choice for $\Phi$ is a sum of pair-wise interactions:

$$
\Phi\left(\mathbf{r}_{1}, \ldots, \mathbf{r}_{N}\right)=\sum_{i=1}^{N} \sum_{j>1}^{N} \phi\left(\left|\mathbf{r}_{i}-\mathbf{r}_{j}\right|\right)
$$


There are a few different potential energy functions. Some commonly used interaction models are the CHARMM (Chemistry at HARvard Macromolecular Mechanics) potential energy function \{Brooks, 1983\}, Amber and Gromos which is used in Gromacs. Potential energy functions consider the summation of the bonded, $E_{\text {bonded }}$ and non-bonded energies, $E_{\text {non-bonded, as }}$ a function of the atomic positions, $\mathbf{r}$. The bonded energies include bond stretching, bond angle bending, and bond rotation. The non-bonded energies account for the interactions between non-bonded atoms separated by 3 or more covalent bonds.

$$
V(\mathbf{r})=E_{\text {bonded }}+E_{\text {non-bonded }}
$$

The $E_{\text {bonded }}$ term is a sum of three terms:

$$
E_{\text {bonded }}=E_{\text {bond-stretch }}+E_{\text {bond-bend }}+E_{\text {bond-twist }}
$$




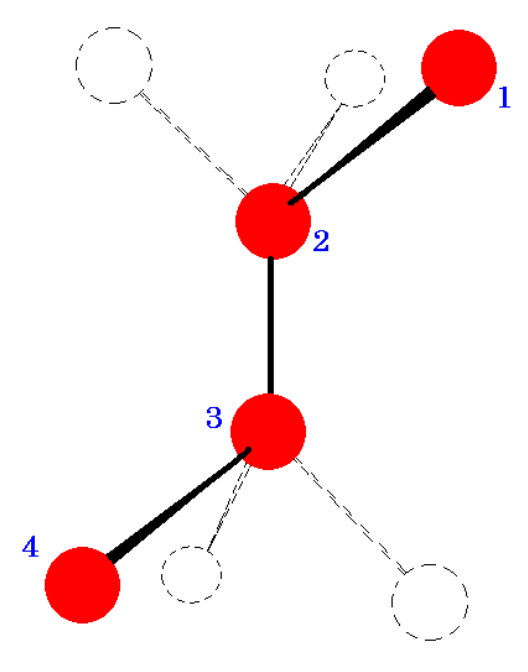

Figure 3.1: Diagram of bond pairs: bond stretching occurs between 1,2 type pairs, bond angle bend occurs between 1,3 type pairs and bond twist occur between 1,4 type pairs.

which correspond to three types of molecular motion. The first term in Equation 3.5 is a harmonic potential representing the interaction between atomic 1,2 pairs where atoms are separated by one covalent bond. The phrase harmonic potential is used to describe the oscillatory behavior of such a system where two masses are separated by a spring with an equilibrium position. The approximation of the energy of a bond as a function of displacement from the ideal bond length, $\mathrm{b}_{0}$, is shown in Equation 3.6. The force constant, $\mathrm{K}_{\mathrm{b}}$, depends on the atoms bonded and therefore determines the stiffness of the bond.

$$
E_{\text {bond-stretch }}=\sum_{1,2 \text { pairs }} K_{b}\left(b-b_{0}\right)^{2}
$$




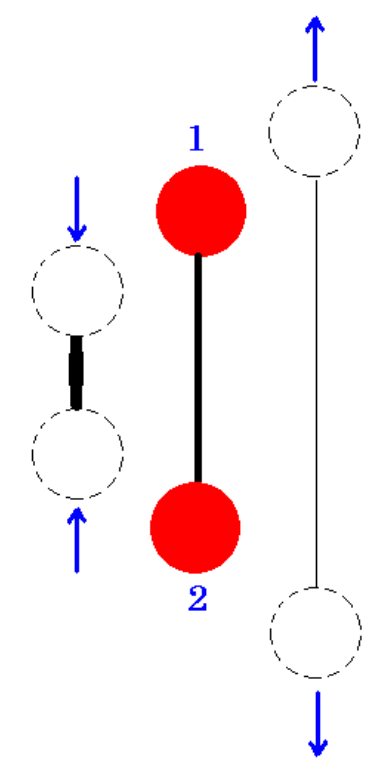

Figure 3.2: Diagram of bond stretching.

The second term in the $E_{\text {bonded }}$ equation is a harmonic potential associated with alteration of bond angles, $\theta$, from the ideal value, $\theta_{0}$, where the values of $\theta_{0}$ and $K_{\theta}$ depend on the chemical type of atoms constituting the angle. This potential occurs between atoms that are separated by 2 covalent bonds, also known as 1,3 pairs.

$$
E_{\text {bond-bend }}=\sum_{1,3 \text { pairs }} K_{\theta}\left(\theta-\theta_{0}\right)^{2}
$$




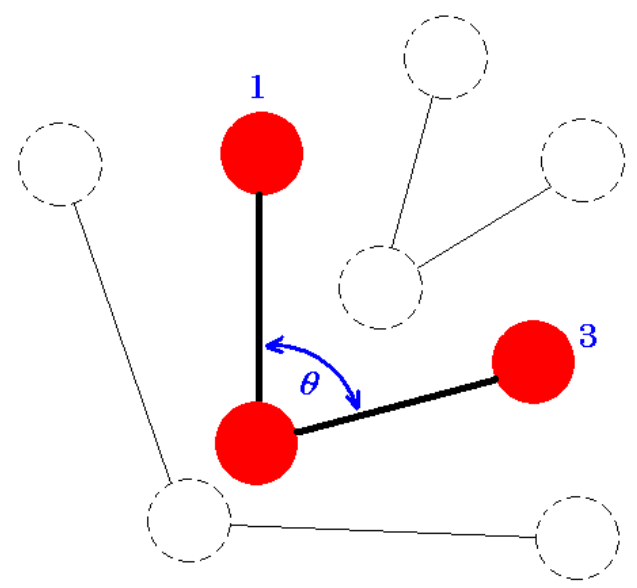

Figure 3.3: Diagram of bond angle bending.

The last term represents the torsion angle potential function between atoms separated by 3 covalent bonds also known as 1,4 pairs. The motion associated with this term is a rotation described by a dihedral angle, $\varphi$, and coefficient of rotational symmetry, $n=1,2,3,4$ or 6 , where rotational symmetry of order $n$ means a rotation by an angle of $360 \%$ does not change the object.

$$
E_{\text {bond-twist }}=\sum_{1,4 \text { pairs }} K_{\varphi}(1-\cos (n \varphi))
$$




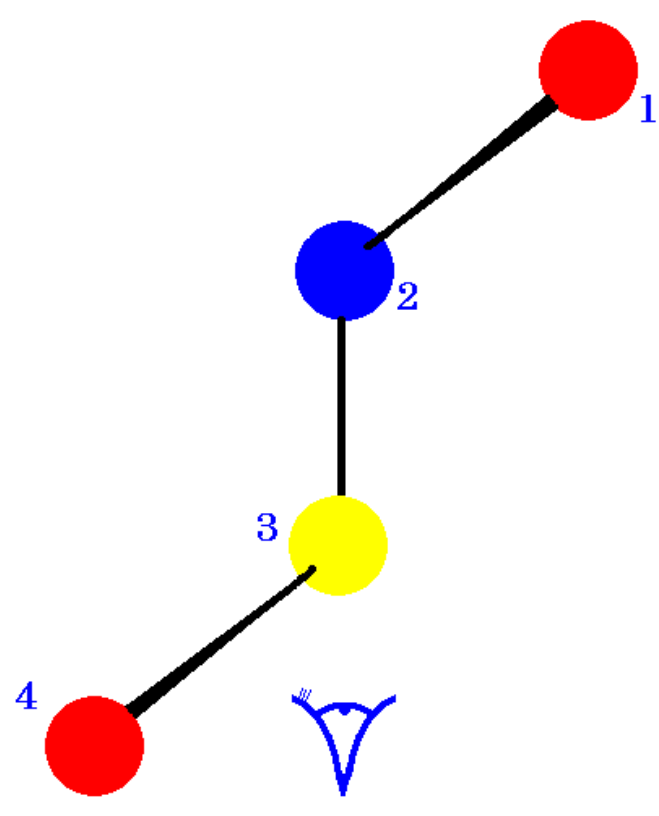

(a)

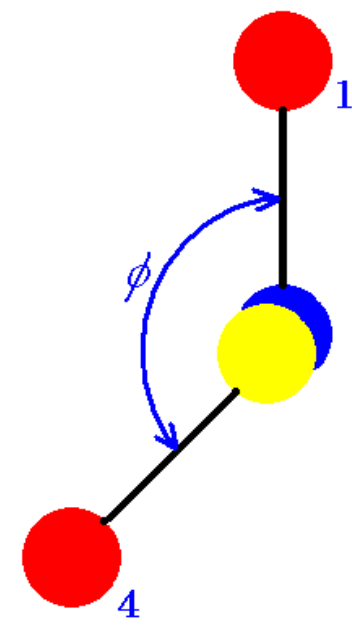

(b)

Figure 3.4: Diagram of bond twisting. View (b) is the perspective of looking down the 3,2 bond of view (a).

The non-bonded energy, $\mathrm{E}_{\text {non-bonded, }}$ is made up of two main components, the Van der Waals interaction energy and the electrostatic interaction energy. Some other potential energy functions also include a term accounting for hydrogen bonds, however in some potential energy functions these interactions are accounted for in the Van-der-Waals and electrostatic terms.

$$
E_{\text {non-bonded }}=E_{\text {Van-der-Waals }}+E_{\text {electrostatic }}
$$


The Van der Waals interaction between two atoms arises from a balance between repulsive and attractive forces. The repulsive forces occur when two atoms are near each other and the electron-electron interaction is strong. The attractive forces, sometimes known as dispersion forces, arise from the fluctuations in the charge distribution in the electron clouds. Fluctuations in one atom or molecule gives rise to an instantaneous dipole, which in turn induces a dipole in the second atom or molecule, therefore stimulating an attractive interaction. The Van der Waals interaction is most often modeled using the Lennard-Jones $12-6$ potential which expresses the interaction energy using the atom-type dependant constants $A$ and $C$ SStote, 1999$\}.$

$$
E_{\text {Van-der-Waals }}=\sum_{\substack{\text { nonbonded } \\ \text { pairs }}}\left[\frac{A}{\mathbf{r}^{12}}-\frac{C}{\mathbf{r}^{6}}\right]
$$

The Lennard-Jones 12-6 potential function is characterized by attractive forces at long range, but as the distance becomes short the repulsive interaction becomes dominant. This give rise to a minimum in energy, $E^{*}$, where the positioning of the atoms at the optimal distance, $r^{*}$, stabilizes the system as seen in Figure 3.5. 


\section{Lennard-Jones Potential Energy Plot}

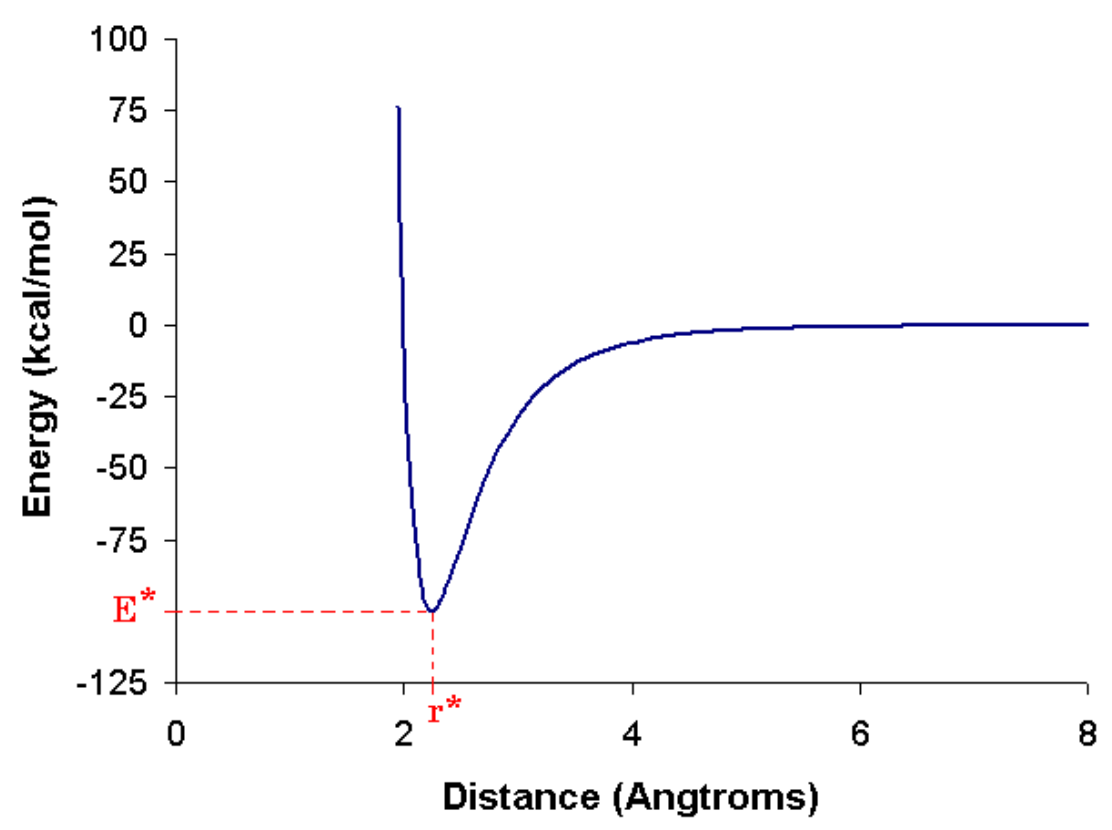

Figure 3.5: Lennard Jones 12-6 potential curve

The last term in the potential energy function accounts for the electrostatic interactions between pairs of atoms represented by Coulomb potential.

$$
E_{\text {electrostatic }}=\sum_{\substack{\text { nonbonded } \\ \text { pairs }}} \frac{q_{1} q_{2}}{\varepsilon_{\text {eff }} d}
$$

Where $\mathcal{E}_{\text {eff }}$ is the effective dielectric constant for the material separating the two atoms and $d$ is the distance between two atoms having charges $q_{1}$ and $q_{2}$. 
The dielectric constant is sometimes known as the relative permittivity and is defined as the ratio of the amount of stored electrical energy when a potential is applied, relative to the permittivity of a vacuum. Permittivity relates to a material's ability to transmit an electric field.

\subsubsection{Global MD Algorithm}

To summarize the information given in section 3.1.1 and 3.1.2 the overall MD algorithm is shown below. In general the MD algorithm calculates the potential, positions and velocities of all the atoms in the system then repeats steps 2, 3 and 4 for the prescribed number of steps. Steps 2, 3 and 4 compute the forces, update the potential, positions and velocities and output the data. 


\section{THE GLOBAL MD ALGORITHM}

\section{Input initial conditions}

Potential interaction $\Phi$ as a function of atom positions

Positions $r$ of all atoms in the system

Velocities $v$ of all atoms in the system

Repeat steps 2, 3, and 4 for the required number of steps:

\section{Compute forces}

The force on any atom

$$
\mathbf{F}_{i}=-\frac{\partial \Phi}{\partial \mathbf{r}_{i}}
$$

is computed by calculating the force between non-bonded atom pairs:

$$
\mathbf{F}_{i}=\sum_{j} \mathbf{F}_{i j}
$$

plus the forces due to bonded interactions (which may depend on

$1,2,3$ or 4 atoms), plus restraining and/or external forces.

The potential and kinetic energies and the pressure tensor are computed.

\section{Update configuration}

The movement of the atoms is simulated by numerically solving

Newton's equations of motion

$$
\begin{gathered}
\frac{d^{2} \mathbf{r}_{i}}{d t^{2}}=\frac{\mathbf{F}_{i}}{m_{i}} \\
\frac{d \mathbf{r}_{i}}{d t}=\mathbf{v}_{i} ; \quad \frac{d \mathbf{v}_{i}}{d t}=\frac{\mathbf{F}_{i}}{m_{i}} \\
\downarrow
\end{gathered}
$$

\section{Output step}

Write positions, velocities, energies, temperature, pressure, etc. 


\subsubsection{Treatment of non-bonded energy terms}

The calculation of the non-bonded energy terms is the most computationally expensive part of a molecular dynamics simulation. In theory, the interaction between every pair of atoms on the system should be accounted for, but the number of calculations increases as the square of the number of atoms for a pairwise model \{Rosenberg, 1982\}. In order to speed up the computation, the interactions between two atoms separated by a distance greater than a predetermined cutoff distance are ignored. There are a few ways to define how to terminate the interaction of a pair of atoms; they are plotted in Figure 3.6. 


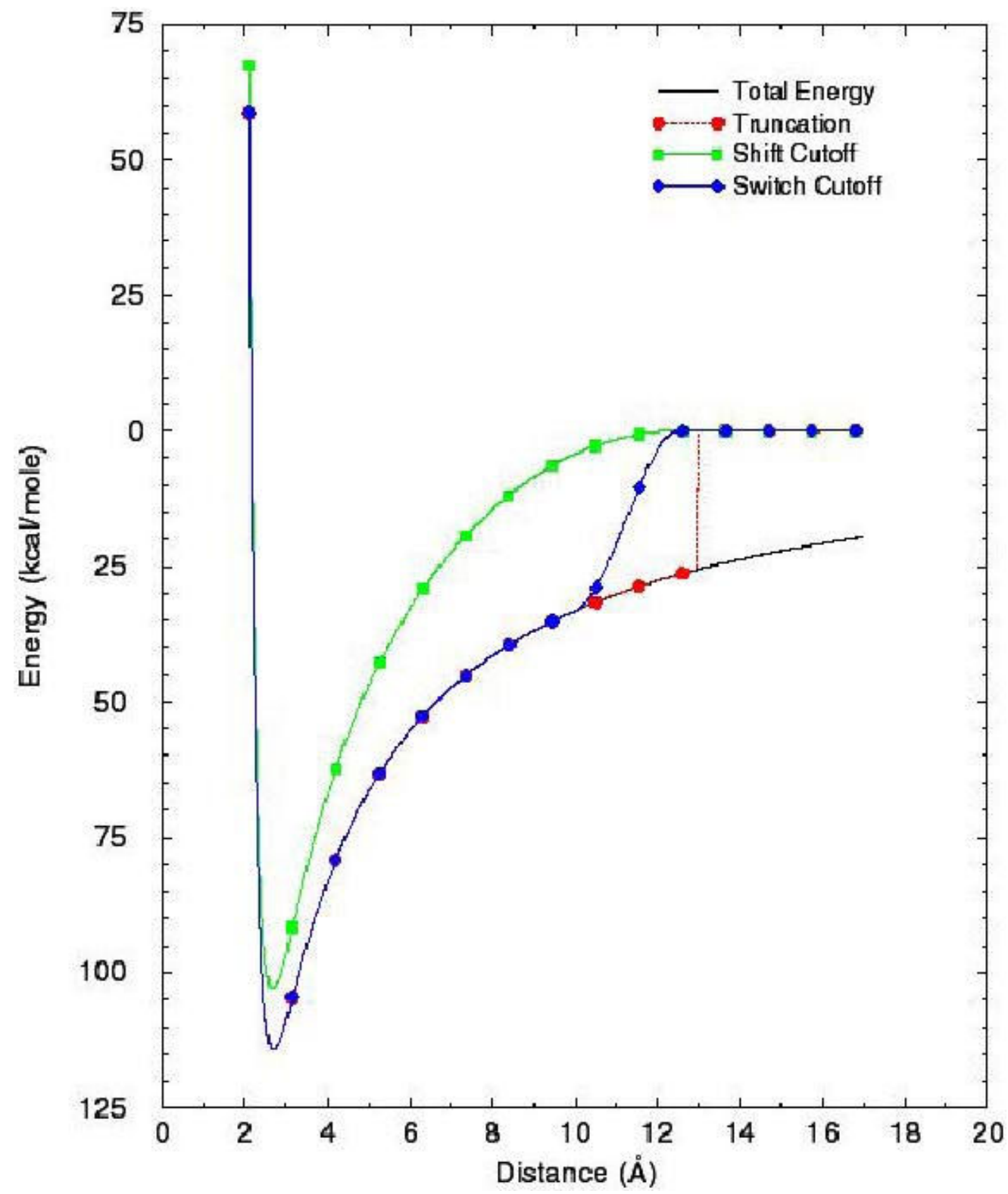

Figure 3.6: Three different methods for defining the cutoff region

In the truncation method all interactions are set to zero for interatomic distances greater than the cutoff distance. The shift cutoff method modifies the entire potential energy surface such that at the cutoff distance the 
interaction potential is zero. The third technique is called the switch cutoff method. This method tapers the interaction potential over a predetermined range of distances. The potential takes its usual path up to the first cutoff distance and is then switched to zero between the first and last cutoff.

For the simulations in this study the switch method was used because it has been shown in previous studies to be stable and have a high correlation to experimental data \{Garemyr, 1999\}.

\subsubsection{Treatment of solvent}

Solvent, typically water, has a fundamental influence on the structure, dynamics and thermodynamics of biological molecules. One of the most important functions of the solvent is the screening of electrostatic interactions. Although water is a neutral molecule it's shape creates a dipole separating into a positive and negative end. This causes electrostatic interactions that should not be ignored. It is important to include solvent effects in molecular dynamics simulations of biological tissues, whether it's implicit or explicitly defined because many of the atomic interactions are with water molecules. Without water being at least implicitly included in the simulation the molecule is essentially simulated in a vacuum with no dielectric constant and therefore no electrostatic charge screening \{Solmajer, $1991\}$. 
The simplest method is to just include a dielectric screening constant in the electrostatic term of the potential energy function. In this implicit treatment the solvent water molecules are not included in the system but an effective dielectric constant is used. Often a distance dependant dielectric

constant is used, $\left(\varepsilon_{\text {eff }}=r_{i j} \varepsilon\right)$ where $\varepsilon$ ranges from 4 to 20 . Although this is a crude approximation it is still much better than using unscreened partial charges \{Stote, 1999\}. Many other implicit solvent models have been developed that range from relatively simple distance-dependant dielectric constants to models that base the screening on the solvent exposed surface area of the protein $\{$ Roux, 1999\}.

In the explicit model the water molecules are explicitly included in the system, therefore the dielectric constant is set to one $(\varepsilon=1)$ in the potential energy function. In this more detailed treatment of the solvent, boundary conditions must be imposed to prevent the water molecules from diffusing away from the system. One popular method for boundary treatment is called periodic boundary conditions.

\subsubsection{Periodic Boundary Conditions}

The periodic boundary condition enables a simulation to be run using a relatively small amount of particles in such a way that the particles 
experience forces as if they were in a bulk solution. This is a classic way to minimize edge effects. For example see Figure 3.7, where eight twodimensional boxes are assembled around the central box of interest. The particles in the neighboring boxes are called image particles and their coordinates are related to those in the primary box by simple translation.

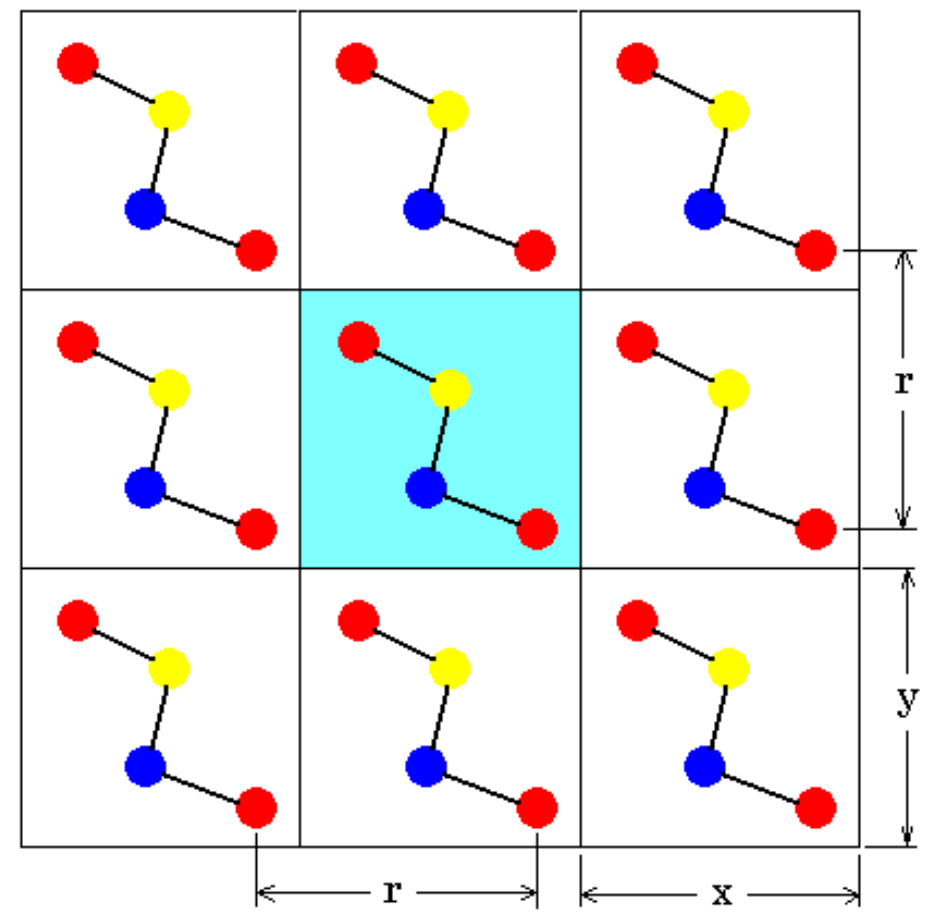

Figure 3.7: Two-dimensional Periodic Boundary Condition. The blue tinted box represents the primary box which is surrounded by image boxes where $x$ and $y$ are the box vectors and $r$ is the distance between an atom and its translated copy.

The simplest box is cubic but some other options include rhombic dodecahedron and truncated octahedron. Typically the image boxes are set 
up in a three-dimensional format, so the primary box is surrounded by 26 neighboring boxes. Forces on the primary particles are calculated from particles within the primary box as well as the image boxes. Atomic interactions are calculated up to a distance called the cutoff distance which is prescribed by the user. The cutoff is chosen such that a particle or atom in the primary box does not see its image in the neighboring boxes. Therefore the cutoff distance must be less than each of the box vectors $x, y$ and $z$.

Usually the box used is determined by the general shape of the molecule under study. In this study a rectangular brick like box is used because it supports the shape of the long chain like GAGs while not leaving too much empty space surrounding them. 


\section{Chapter 4}

\section{Methods}

\subsection{Aims}

The objective of this study is to develop a molecular-based nanomechanical model of cartilage proteoglycans for several different mechanisms that may be related to changing GAG density. Molecular mechanics methods will be used to obtain pressure, work and density data for chondroitin sulfate GAGs for these mechanisms. A more descriptive explanation on these mechanisms will be given in sections 4.1.1-4.1.3. The long-term outcome of this aim is the development of molecular-based nanomechanical models of cartilage proteoglycan solutions and the refinement of the finite deformation constitutive equations used in a Cartilage Growth Model (CGM). 


\subsubsection{Single Chain Simulation}

An experiment with single GAG chains will be studied in order to choose a length or number of disaccharide units to use in the molecular dynamics simulations. This is important because the size of the system should be short enough to minimize the computing resources but long enough to avoid boundary conditions like end effects and chain curvature. Figure 4.1 shows an example of the set up of one of these simulations.

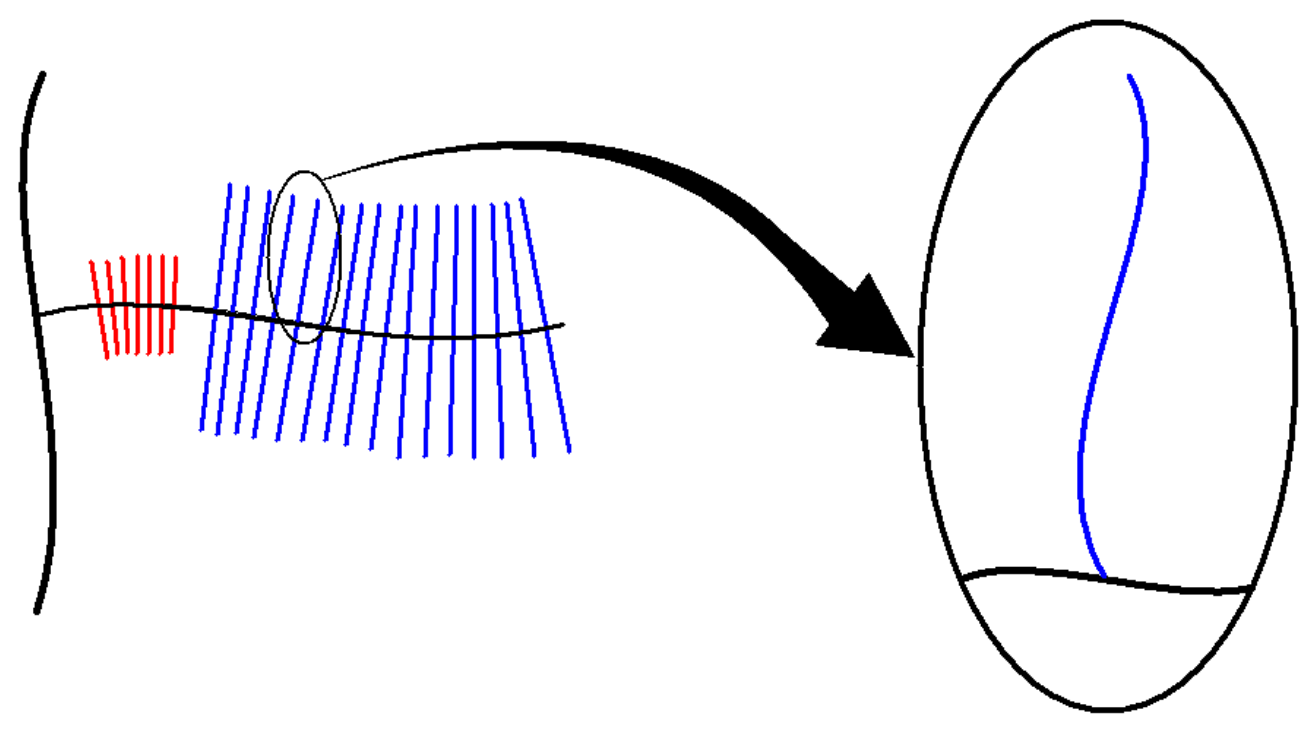

Figure 4.1: Single chain simulation set up with the bottom of the chain fixed and the rest allowed to move and find equilibrium conformation. 


\subsubsection{Five Chain Simulation}

Another simulation will be investigated to find the correlation between GAG density and pressure on the system. These results will be compared to the Donnan model \{Maroudas, 1979\} and Poisson-Boltzmann unit cell (PB) model \{Marcus, 1955\}. Five chains will be used in a 3-D pattern to imitate a physiological situation where aggrecan molecules are in close contact and many GAG chains are close together. Figure 4.2 shows the physical relevance to this study.

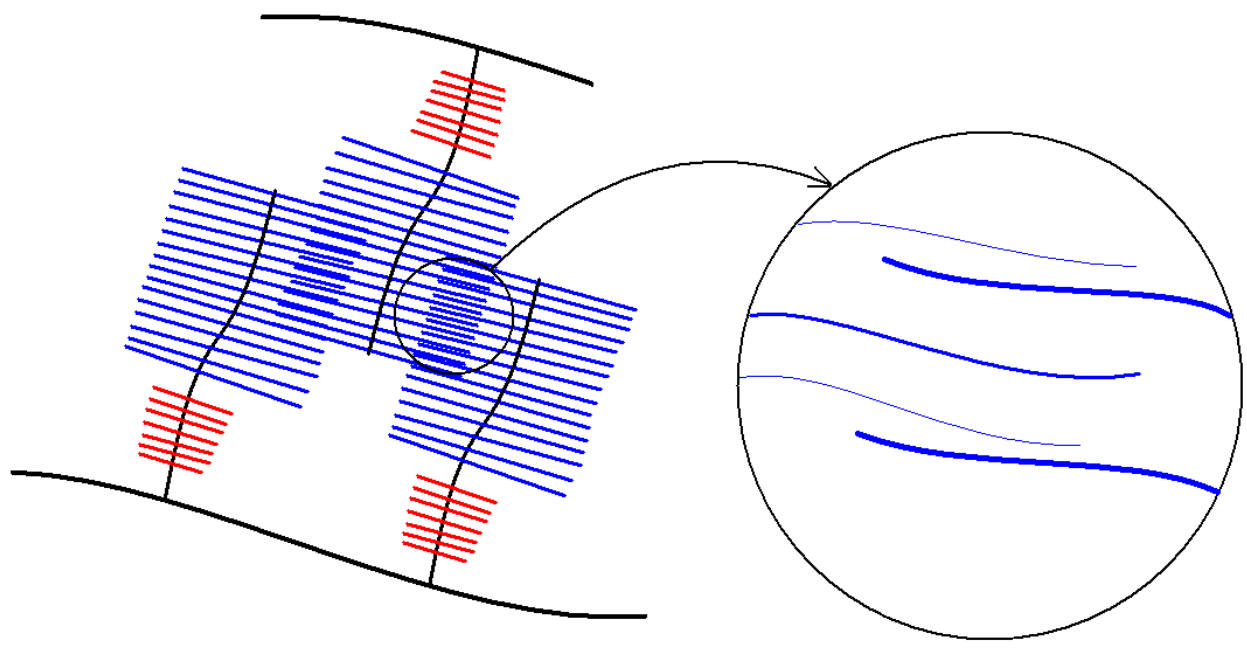

Figure 4.2: Physical relevance of the five chain simulations. 


\subsubsection{Pulling Simulation}

The last experiment is designed to mimic a physiological scenario where one GAG chain is dragged between two adjacent parallel chains seen in Figure 4.3. This simulation will give insight into the forces involved to compress two aggregated groups of GAG chains together.

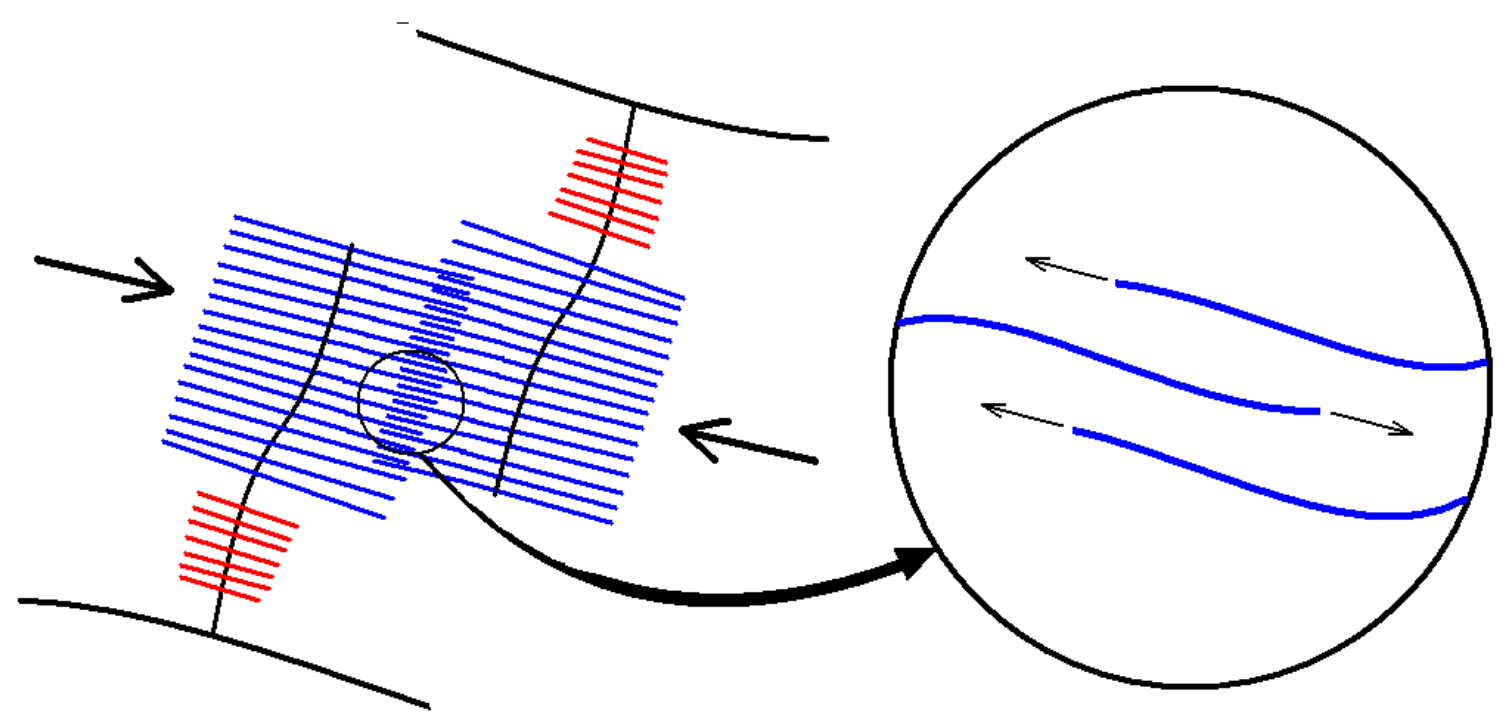

Figure 4.3: Physical situation where a GAG chain is pulled between adjacent GAG chains. Rate of pulling is $0.01 \mathrm{~nm} /$ pico-second. $^{-}$ 


\subsection{Models}

All models and simulations were created using the software GROMACS version 3.3. GROMACS is a program for performing molecular dynamics simulations and energy minimization using molecular modeling. Molecular modeling is described as the process of representing complex chemical systems in terms of a realistic atomic model with the goal of attaining macroscopic properties based on detailed knowledge on a nano-scale.

\subsubsection{Force Field}

The force field is what contains the sets of equations for the potential functions described in section 3.1.2. Also included in the force field are all the parameters used in these equations. Information on the atoms and atom combinations is pulled from the topology file and used in the potential functions to calculate potential energies and their derivatives, the forces.

\subsubsection{Topologies}

In order for GROMACS to use the potential functions in the force field it needs to know on which atoms and combinations of atoms to act. It must also 
know what parameters to apply to the various functions. All this is defined in the topology file, which lists the constant attributes of each atom. Information such as atom type, mass, and charge are included. Atom combinations are also listed in the topology file, such as bond pairs, bond angle groups and bond dihedral groups. Depending on the atoms involved in the group different constants are used in the force field potential functions.

\subsubsection{Water and Ions}

The size of the periodic boundary box will determine how many explicitly defined water molecules will be in the system. Having too large of a box and therefore too many water molecules can slow a simulation considerably because Gromacs calculates all the bonded and non-bonded interactions of every atom in every water molecule. All simulations in this study use explicitly defined water in the form of the Single Point Charge (SCP) model \{Berendsen, 1981\}.

When studying molecules at the nano level one must decide how to include ions. For the simulations in this study a total net charge of zero is used because of the small scale. A hypothesis that could be explored in a future study is that over enough time positive and negative ions would flow in and out of a small area to create a zero net charge in any given location. The overall ionic concentration in the extra cellular matrix is $0.15 \mathrm{M} \mathrm{NaCl}$ 
\{Basser, 1998\}, but concentrations from 0.01 to $1 \mathrm{M}$ are often studied \{Buschmann, 1995\}. A derivation of the ionic concentration of the models used in this study is shown in Appendix A.

\subsection{Simulations}

Three simulations were considered to discover the compressive behavior of GAGs. These simulations were chosen because they mimic a physiological situation that would exist during compression of AC. There are many options when choosing a physical state of GAGs under compression. These particular states were chosen for their simplicity but also because they represent our hypothesis for several kinematic mechanisms for the compression of GAGs.

\subsubsection{Single Chain Simulations}

First a length of GAG, or number of CS units, must be chosen to use for subsequent simulations. A model of one chain is simulated by itself, only varying the length of the chain in each test. The goal behind this test is to find a length that is long enough to avoid unwanted boundary or end effects but short enough to remain computationally inexpensive. Four different lengths were tested: 5, 10, 15 and $20 \mathrm{CS}$ units. A simple schematic of the simulation set up is shown in Figure 4.4. 


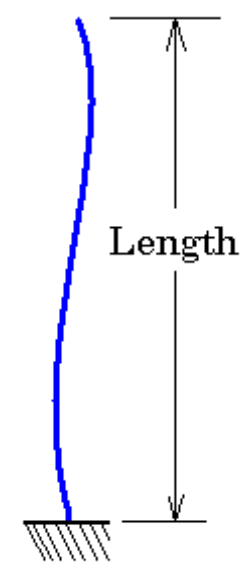

Figure 4.4: Set-up for the single chain simulations. The bottom of the chain is fixed and the rest of the chain is allowed to move freely.

The general bending and stretching motion of the GAG chains is the result of interest. In order to quantify this, the distance between the distal and proximal chains ends will be calculated at each time step of the simulation. That data will be plotted and considered when choosing the optimal chain length. In general, the less bending and stretching a molecule undergoes during a molecular dynamics simulation the more stable it is. Therefore the standard deviation of the end to end length will be crucial for determining the most stable chain length. 


\subsubsection{Five Chain Simulations}

Once a standard is set for the length of GAG to be used in the rest of the simulations the next step is to include multiple chains in a simulation. Five GAG chains were used because of the 3-dimensional arrangement that can be employed. A schematic showing the set up for this simulation is shown in Figure 4.5.

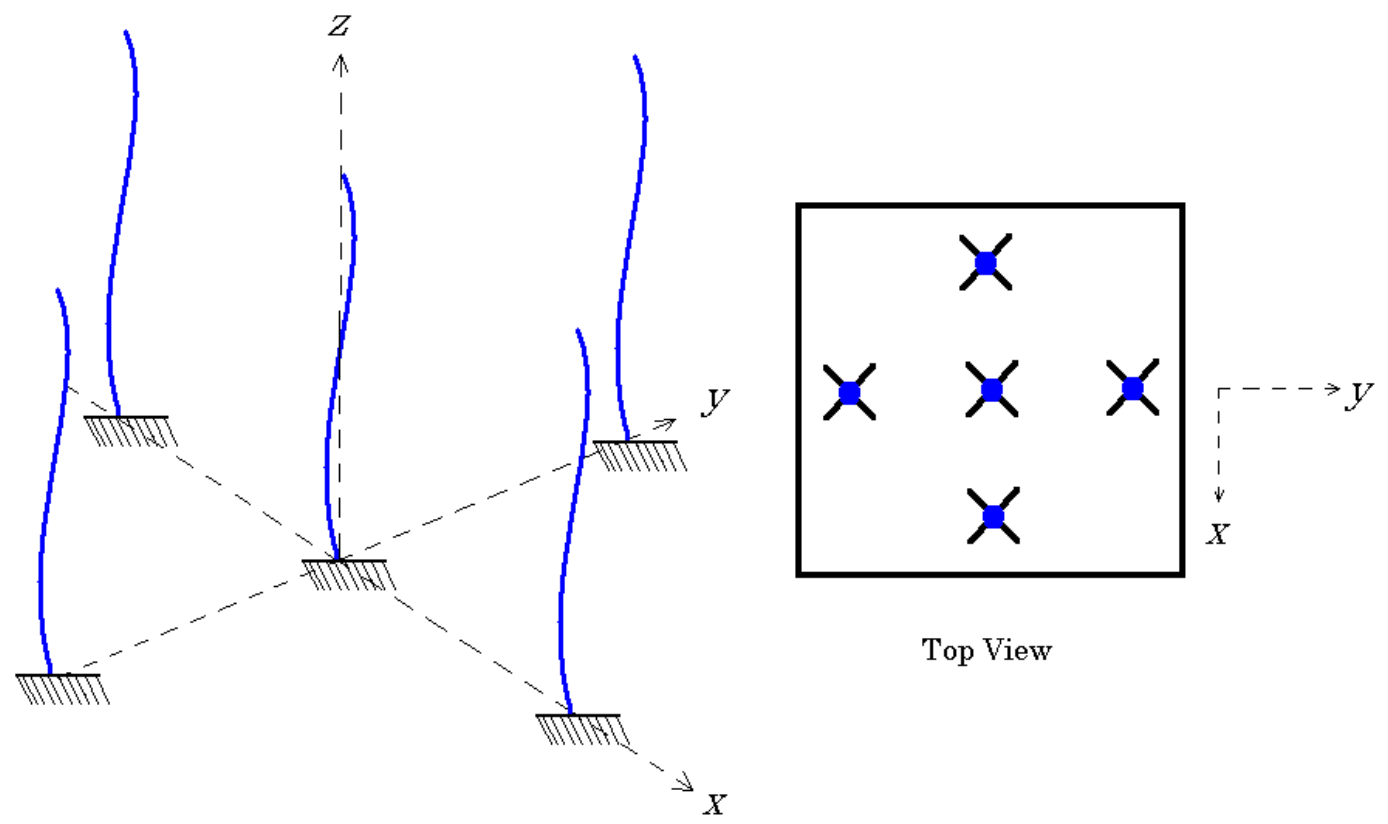

Figure 4.5: Five chain simulation set up. A top view is shown in the box on the right to reveal the 3 -dimensional nature of the simulation arrangement.

In this simulation the only varied property is the distance between chains. As the distance between the chains change so does the periodic 
boundary box size and as a result the density is varied. With the size of these systems and the computational power available a simulation of 1.5 nano-seconds was evaluated.

The goal for this simulation is to output the pressure inside the box for each density of GAGs and compare it to the Donnan \{Maroudas, 1979\} and PB cell model \{Marcus, 1955\}.

\subsubsection{Pull Simulations}

The last simulation in this study mimics a physical situation where two aggrecan molecules are being compressed together and the GAGs are forced into each other. This causes some of the GAG chains to slide in between the others. In order to mimic this situation a model was created with three chains in parallel. The middle chain is then pulled through the two outside chains using a theoretical spring which is a function GROMACS provides in their software. The spring is attached to the CS group at the end of the center chain. The spring constant, direction and velocity are prescribed in the parameter file. The only thing that changes for each case is the spacing between chains. A schematic of the simulation setup is shown in Figure 4.6. 


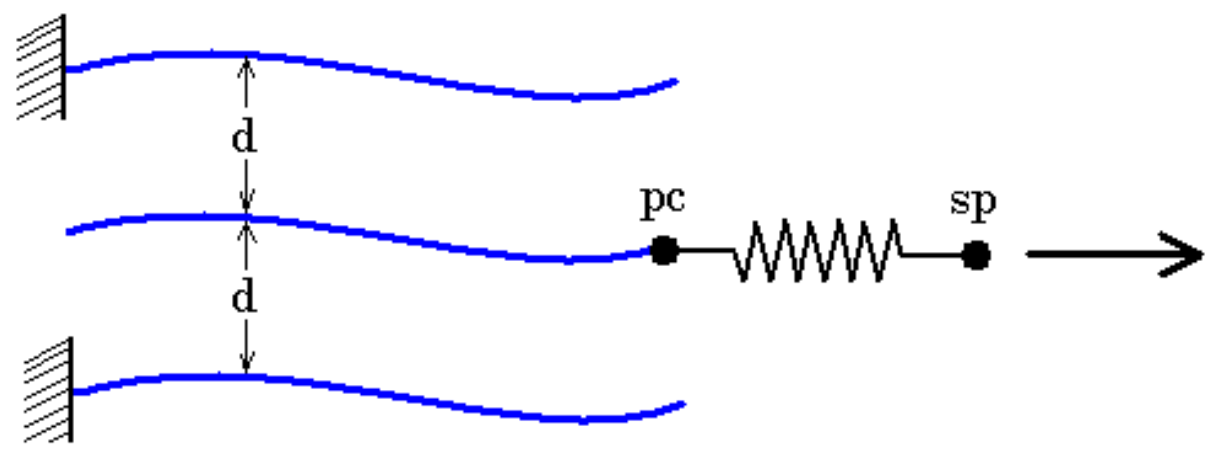

Figure 4.6: Schematic of pull simulation setup including chain spacing, $d$. The desired outcome is the work required to pull the center chain out at four different values of $d$. The output file for the simulation contains the location of pull group, pc, and the location of the spring end, sp. The spring is in equilibrium when the simulation starts and is stretched due to the resistance of the GAG chain being pulled through the others. The force is calculated using the equation for an ideal spring, where $\mathrm{k}$ is the spring constant and $\mathrm{x}_{\mathrm{sp}}$ and $\mathrm{x}_{\mathrm{pc}}$ are the locations of the spring and pull groups in the $\mathrm{x}$-direction.

$$
\mathbf{F}=k_{s p r i n g}\left(\mathbf{x}_{s p}-\mathbf{x}_{p c}\right)
$$

The work done by the spring is:

$$
W=\int \mathbf{F} \bullet d \mathbf{s}
$$


The work is calculated numerically using a simplified version of Equation 4.2 for time-steps, $\mathrm{t}=1$ to $N$.

$$
W=\sum_{t=1}^{N} \mathbf{F}_{t}\left(\mathbf{x}_{t}-\mathbf{x}_{t-1}\right)
$$




\section{Chapter 5}

\section{Results}

\subsection{Single Chain Simulation}

Calculating the distance from the proximal end to the distal end of each chain length during a 2 nano-second simulation shows the relative bending and stretching movement of each chain. See Figure 5.1 for a visual description of the length calculation. The distance is normalized by the number of units in the chain and the motion is shown in Figure 5.2. The curves have different start values because each chain was simulated to a minimized state before running the single chain experiment. 

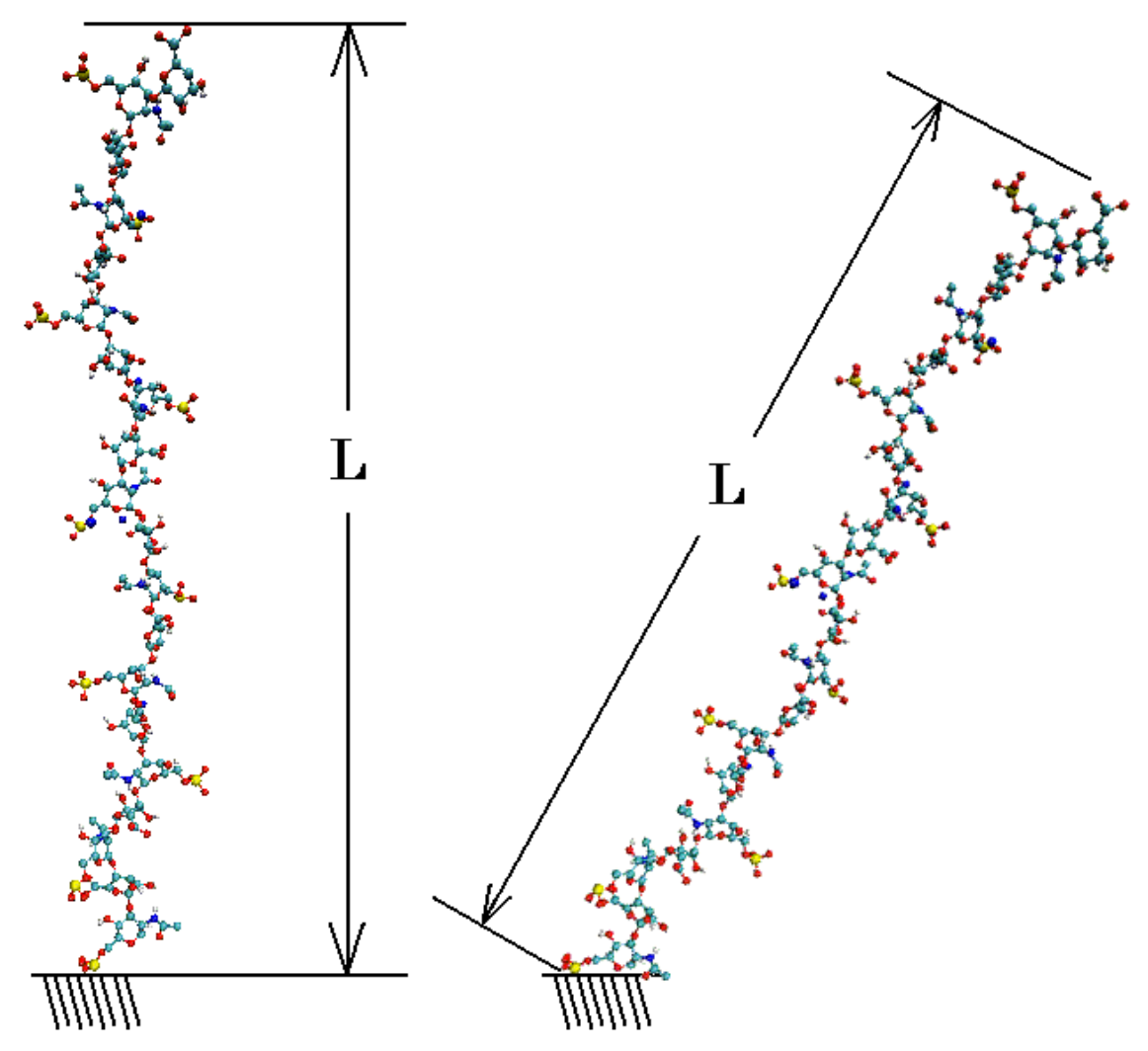

Figure 5.1: This figure shows how the end-to-end length is calculated. It is the vector magnitude from the proximal end to the distal end. 


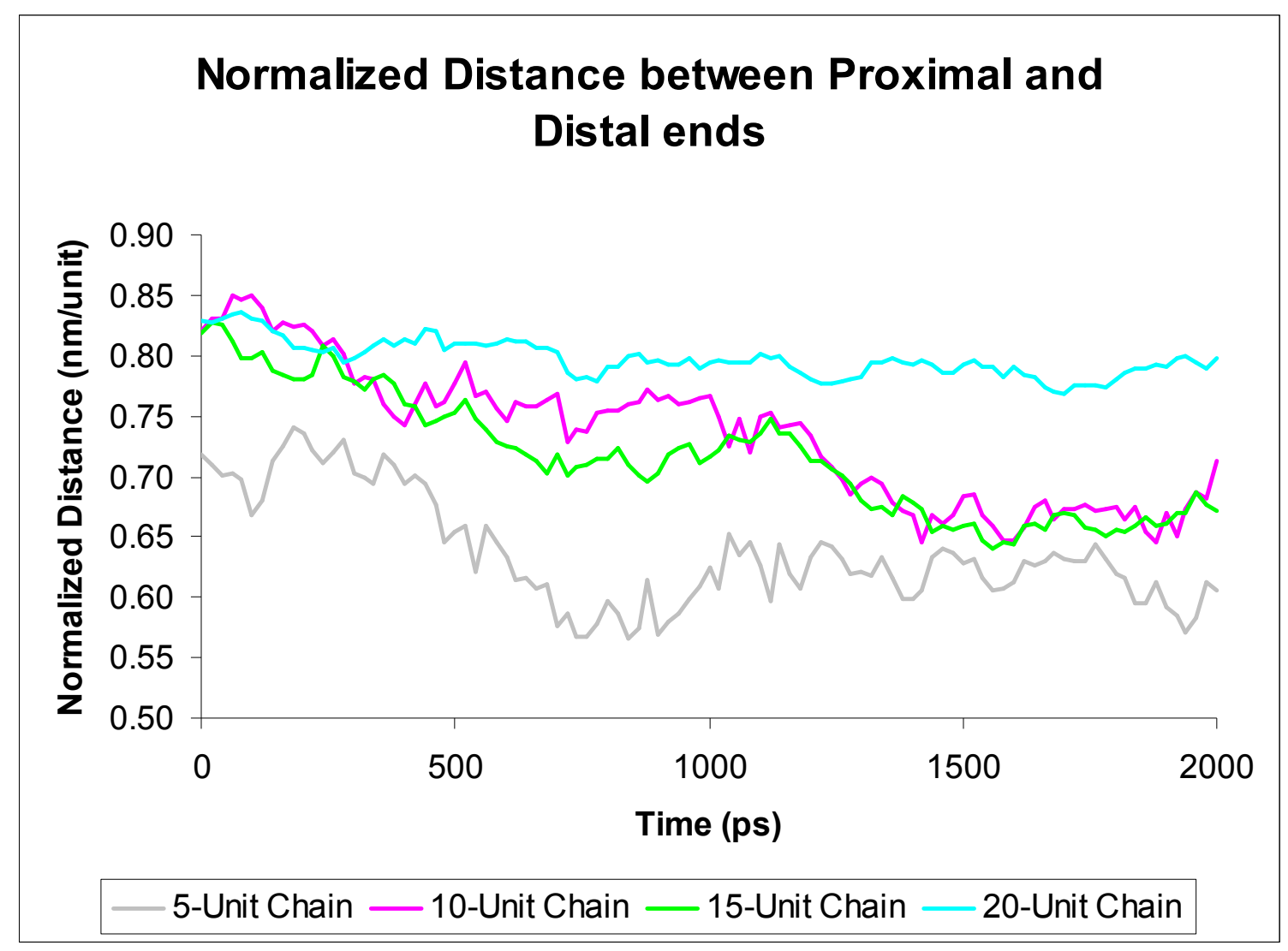

Figure 5.2: Distance from one end of the chain to the other, normalized by the number of CS units. A value of one would represent the chain remaining at it's maximum contour length.

Figure 5.3 shows the standard deviation of the normalized length distance for each chain's motion. The standard deviation of the chain motion is a good method for judging each chain's stability. A lower standard deviation implies less bending and stretching of the chain during a simulation. 


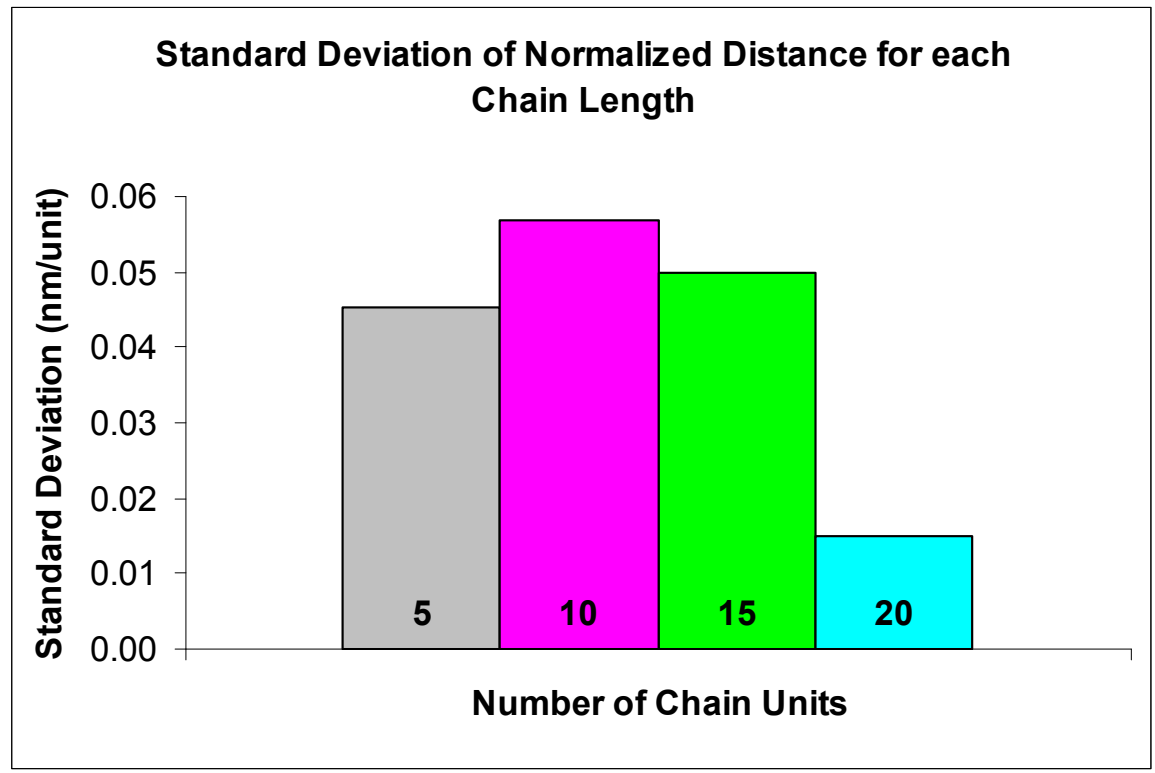

Figure 5.3: Standard deviation of the normalized distance for each chains motion.

The 20-unit chain is obviously the most stable, while there is not a large difference in the standard deviations of the other three chain lengths. The 5 unit chain should be discarded due to visualized end effects and the fact that the persistence length of chondroitin-6-sulphate is about 4 CS units $\{$ Bathe, $2005\}$. The 20-unit chain can justifiably be thrown out because it will create high computational costs when running the 5-chain and pull simulations. There is not a large difference between the standard deviation of the 10 and 15 unit chains so the most reasonable solution is to go with the shorter length to reduce computational costs. Therefore a 10 -unit chain was selected to run the rest of the simulations. 


\subsection{Five Chain Simulation}

The pressure data from the 5-chain simulations is presented in Table 5.1 and Figure 5.4. Figure 5.4 also includes data points from the PB cell model fit and the Ideal Donnan model \{Buschmann, 1995\}. The pressures observed in the 5-chain simulations are qualitatively similar to those in the PB cell and Donnan models except for the point at $179 \mathrm{mg} / \mathrm{ml}$ density. One possible reason why this data point does not fit well with the other models is that it has an ionic concentration of $0.25 \mathrm{M} \mathrm{Na}^{+}$when the overall ionic concentration in the extra cellular matrix is $0.15 \mathrm{M} \mathrm{NaCl}$ \{Basser, 1998\}.

Also, both the 1 and $2 \mathrm{~nm}$ spaced systems are not in equilibrium with the physiological GAG spacing. Recall that the physiological spacing of GAG chains is about $3-4 \mathrm{~nm}\{\mathrm{Ng}, 2003\}$. Therefore the systems at 3 and $4 \mathrm{~nm}$ spacing are already near equilibrium and therefore see pressures near atmospheric pressure $(100 \mathrm{kPa})$. The systems starting with GAGs spaced at 1 and $2 \mathrm{~nm}$ are much closer to each than the physiological spacing. Therefore large pressures are exerted on the walls of the periodic boundary box to allow the molecules to separate. The simulation is set up to allow the volume of the box to grow to maintain atmospheric pressure but it can't do so instantaneously. Given a long enough simulation the systems would 
equilibrate to atmospheric pressure. The pressure shown on Table 5.1 are the pressures exerted on the box by the system as it tries to expand.

Table 5.1: Average pressure data for 5 different GAG densities. Typical GAG spacing is 3-4 $\mathrm{nm}$ \{Ng Grodzinsky, 2003\}.

\begin{tabular}{|c|c|c|}
\hline $\begin{array}{c}\text { Spacing } \\
\text { (nm) }\end{array}$ & $\begin{array}{c}\text { Density } \\
\text { (mg/ml) }\end{array}$ & $\begin{array}{c}\text { Average Pressure } \\
\text { (kPa) }\end{array}$ \\
\hline 1 & 179 & 269 \\
\hline 2 & 87 & 257 \\
\hline 3 & 55 & 48 \\
\hline 4 & 36 & 91 \\
\hline
\end{tabular}

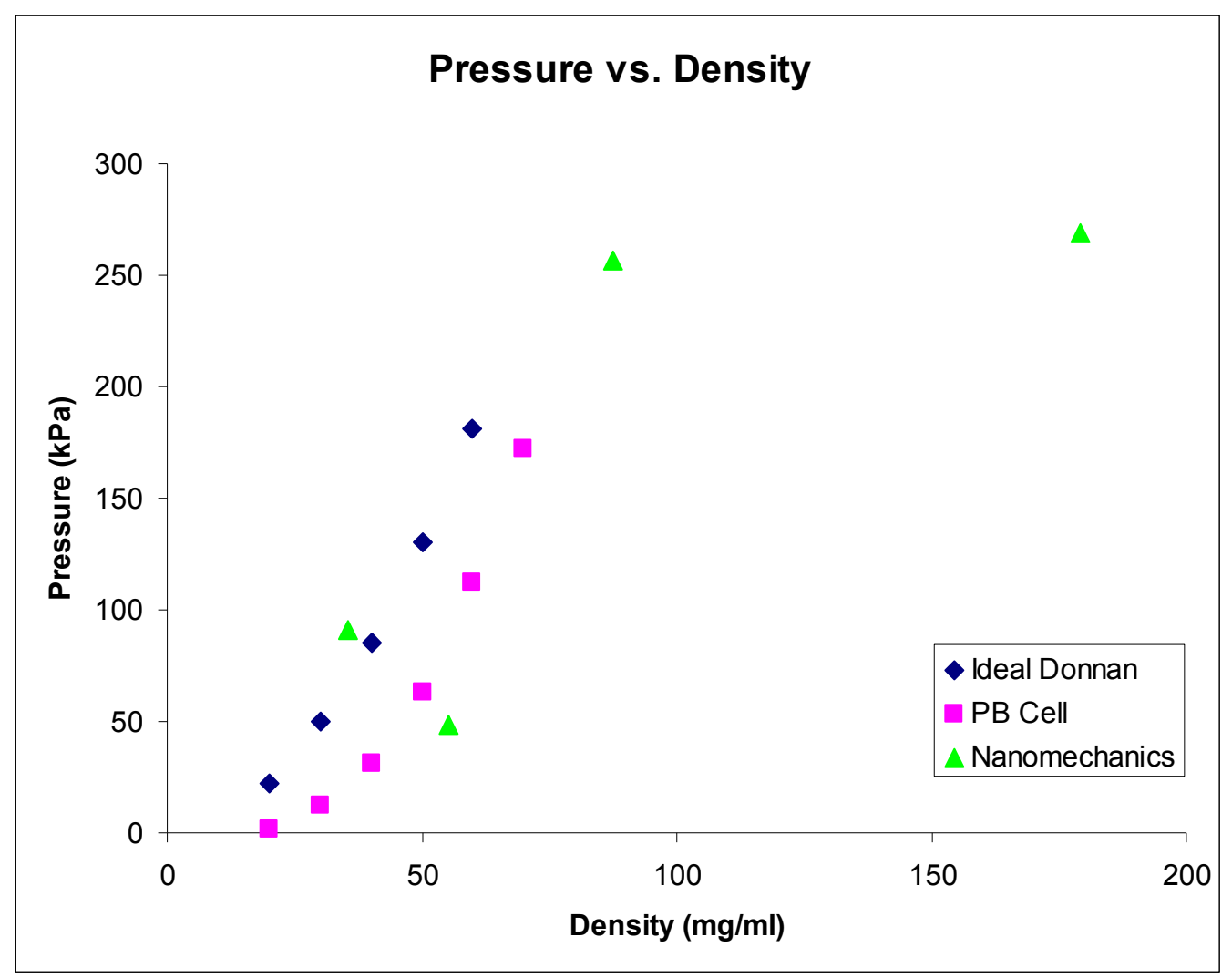

Figure 5.4: Pressure vs. density compared with Donnan and PB cell models. The PB cell model was fit to the data of Williams and Comper. The simulation time was 1.5 nanoseconds. 
Table 5.2: Swelling pressure data from the PB cell model and the Ideal Donnan model.

\begin{tabular}{|c|c|c|}
\hline $\begin{array}{c}\text { Density } \\
\text { (mg/ml) }\end{array}$ & $\begin{array}{c}\text { PB Cell } \\
\text { (kPa) }\end{array}$ & $\begin{array}{c}\text { Ideal Donnan } \\
\text { (kPa) }\end{array}$ \\
\hline 20 & 2 & 22 \\
\hline 30 & 12 & 50 \\
\hline 40 & 31 & 85 \\
\hline 50 & 63 & 130 \\
\hline 60 & 112 & 181 \\
\hline 70 & 172 & - \\
\hline
\end{tabular}

\subsection{Pulling Simulation}

The work to pull the central chain out from between the two outer chains is plotted in Figure 5.7. Physiological AC GAGs are separated from each other on the main protein by $3-4 \mathrm{~nm}$, which corresponds to a value of $d$ equal to $1.5^{-}$ $2 \mathrm{~nm}\{\mathrm{Ng}, 2003\}$. Recall that $d$ is the spacing between chains. The spacing at $4 \mathrm{~nm}$ represents infinite spacing because $4 \mathrm{~nm}$ is far enough that each molecule can not "see" the others. In other words, the molecules are spaced farther than the prescribed cut-off distance and therefore are not included in the calculations for potential.

In general, the data shown in Figure 5.7 suggests that it takes more work to pull chains that are spaced farther apart except in the case when the spacing is 1.5 and $2 \mathrm{~nm}$. Those two values of GAG spacing represent the closest values to physiological AC. Observations from the visual 
representation of the molecular dynamics simulations suggest that the positive ions added to the system are most responsible for how the charge groups on the GAGs react to each other. When there are positive ions between a negative charge group of the central chain and one of the outer chains, it causes an attractive force and as a result takes more work to pull the central chain out. When there are no positive ions between two charge groups they will have a repulsive force and take less work to pull out. In the simulations with a smaller space between GAGs there are less positive ions in between the GAGs and therefore cause less attraction between charge groups. In contrast, when there is more space between GAGs there is more room for the positive ions to get in between negative charge groups and cause attractions that make it more difficult to pull out. Figure 5.5 shows a snapshot of a pulling simulation where you can visually see the strain caused by a positive ion between two negative charge groups. 


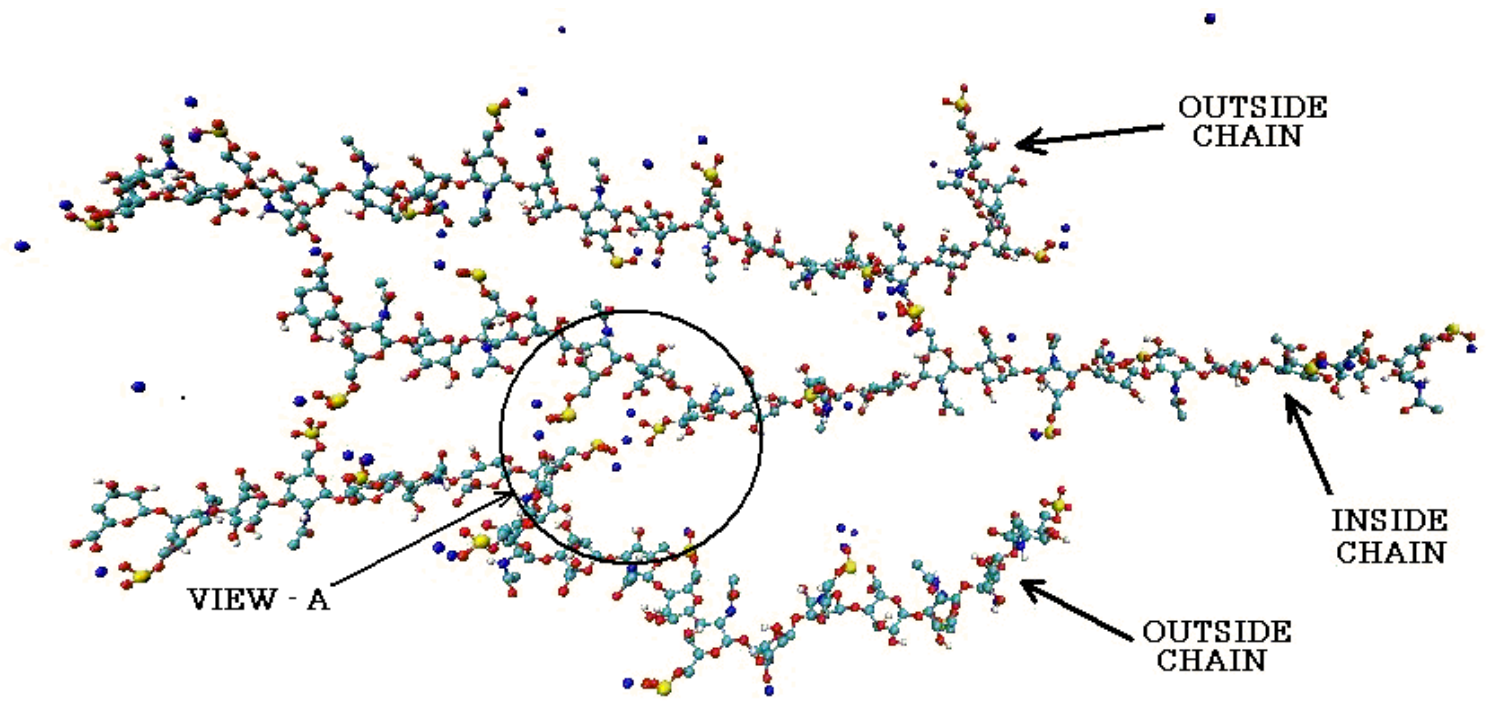

Figure 5.5: Snapshot of pull simulation at $1.5 \mathrm{~nm}$ spacing. See Figure 5.6 for zoomed view - A.

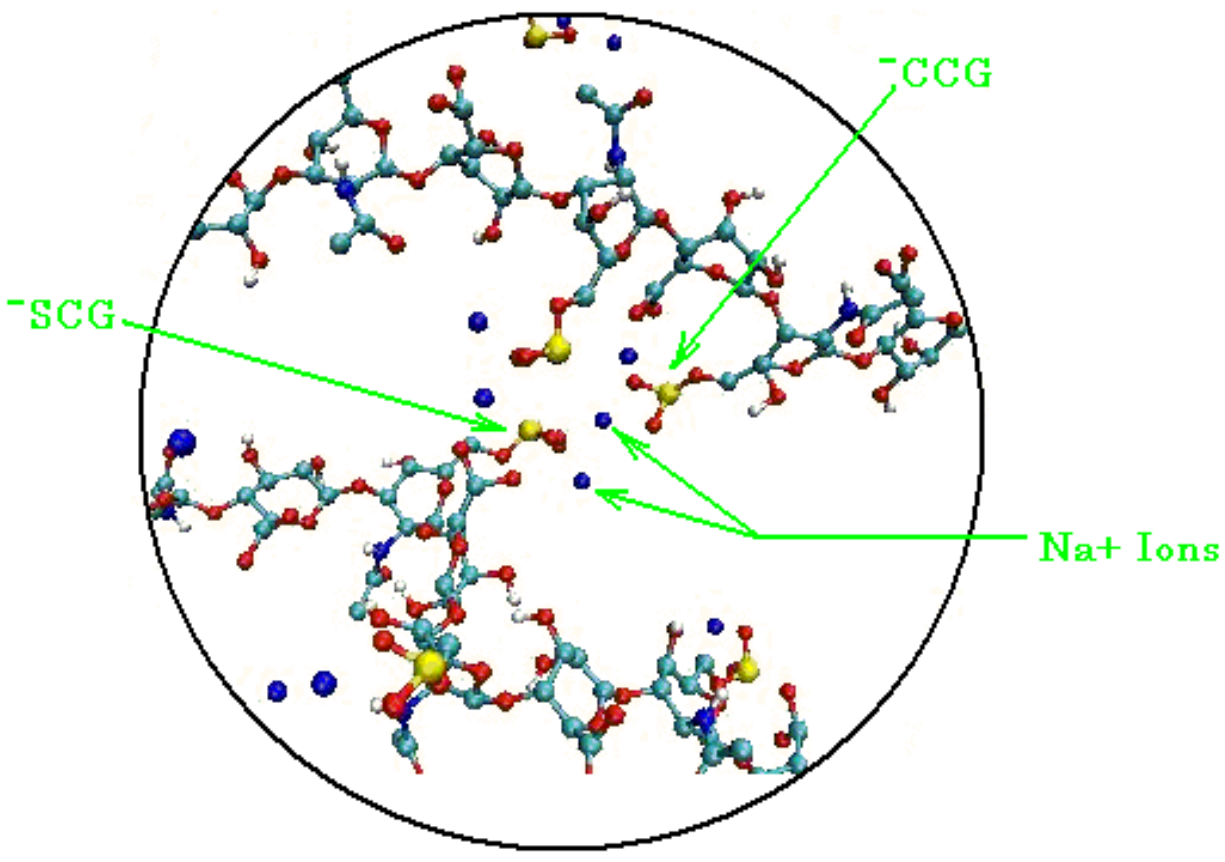

Figure 5.6: View $-\mathrm{A}$ : The dark blue atoms represent $\mathrm{Na}^{+}$ions. The yellow atoms represent Sulfur from the OSO charge groups. $\mathrm{CCG}=$ Central Charge Group; SCG = Side Charge Group 


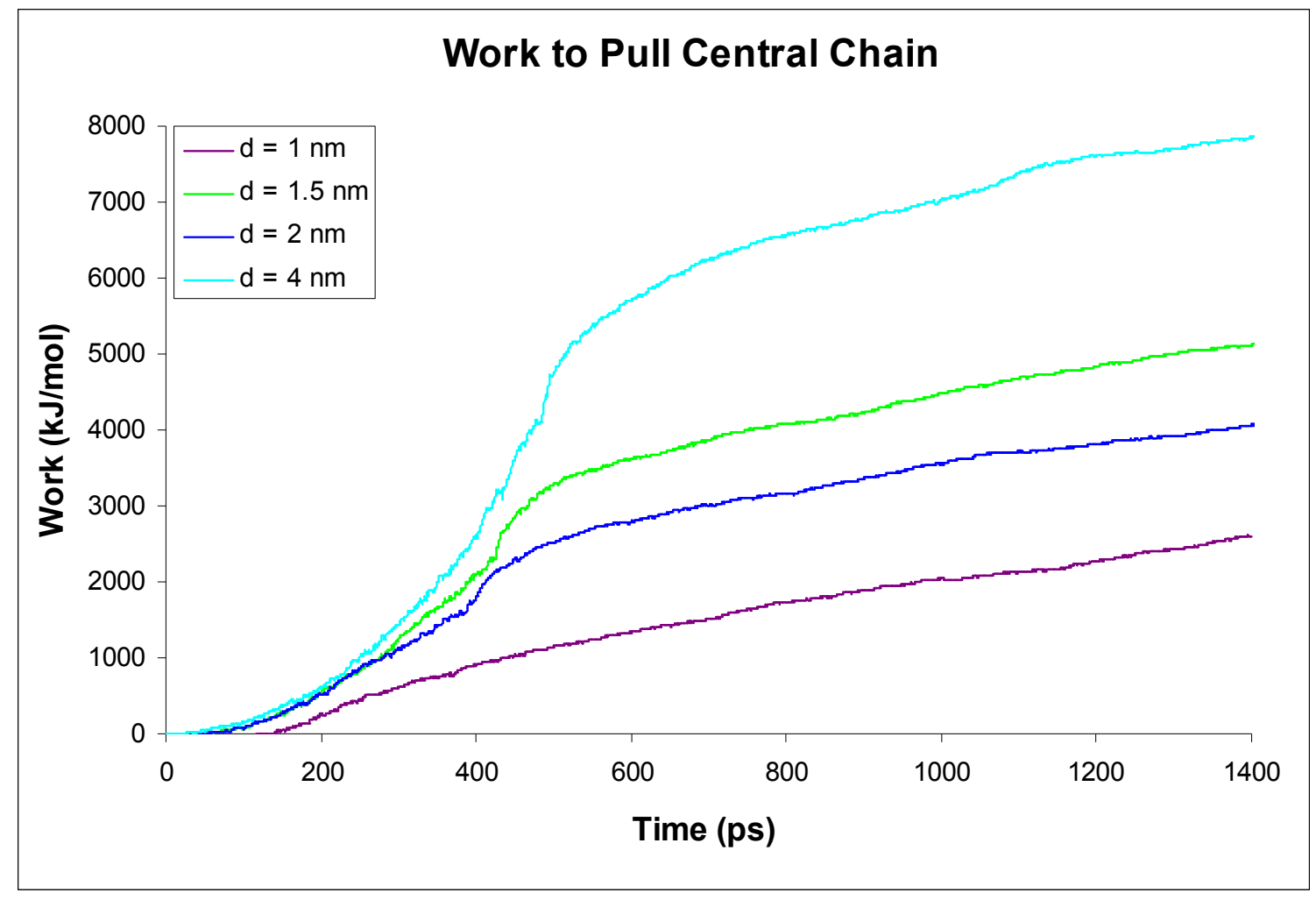

Figure 5.7: Plot of the work required to pull the central chain out from between the two outer chains. Rate of pulling is $0.01 \mathrm{~nm} / \mathrm{pico-second.}$

Using a different ion concentration could greatly affect these results. By altering the amount of ions in the system it will cause some of the attractive or repulsive interactions to change, therefore taking more or less work to pull the central chain. 


\section{Chapter 6}

\section{Conclusion}

\subsection{Discussion}

There are several novel results that came from this study. One is that AC GAGs can be simulated using molecular dynamics simulations and the GAG pressure-density relations obtained are reasonably close to data of microstructural level models. Another significant finding is the role that the positive ions play in the interactions of GAGs.

Although the pressure-density values from the five chain simulations did not follow the exact trends of the PB cell and Donnan models, the values were of the same order. The pull simulations gave us insight into the ways that GAGs interact during a possibly physiological mechanism for changing GAG density. Through visual observations of the molecular dynamics pull 
simulations we witnessed the effect that the positive ions have on GAGs. The positive ions act as a link to bind negative charge groups from one chain to another and make it difficult for the GAGs to slide along each other, thus attributing to the compressive stiffness observed in AC.

The spacing of the GAGs also affects compressive resistance. Interestingly the tighter spacing, $1 \mathrm{~nm}$, did not take the most work. One hypothesis is that this is due to the fact that less positive ions could come between the negative charge groups and act as the linking between the charge groups of the GAGs. The spacing values that are more physiological, $1.5 \mathrm{~nm}$ and $2 \mathrm{~nm}$, took more work to pull which only justifies real spacing of GAGs on aggrecan. In order to get results that will help us better understand the compressive mechanics of GAGs under compression this work must be taken to the next step.

\subsection{Limitations}

One limitation of this work is that pulling was simulated instead of pushing: The aim of this experiment was to study a mechanism that may occur during compression of cartilage. It would be more correct to simulate GAG chains being pushed together instead of pulled from each other. Pushing is much more complicated because of the buckling that would happen to the chains as they are pushed together. For this reason pulling 
was used to get results while keeping the work within the scope of the project.

\subsection{Future Work}

The simulations of single GAG chains provided the selection of a 10 unit GAG chain as the best solution for this study. However, much of the justification was based on the current power and speed of computers and what they can handle in a reasonable amount of time. Computers continue to get faster and more powerful with time and therefore the computational cost of using a longer chain will cease to be a good reason for choosing a shorter one. Therefore it may be in the best interest of future investigators of this molecule to use a 20 unit GAG chain for its stability and proximity to a more physiological GAG chain.

It is very difficult to compare results from simulations with only a few GAG chains to experimental results on a macro scale, therefore the next step will be to scale up this work to the next level. The next level is often called coarse-graining and has already been studied by some authors B Bathe, 2005, Shelley, 2001\}. Coarse graining is method in which not all atoms are explicitly defined in the simulation but "pseudo-atoms" are defined as groups of atoms. This allows for larger systems with more molecules and longer simulation time scales. 


\section{List of References}

Ateshian, G.A., Wang, H., Lai W.M., The role of interstitial fluid pressurization and surface porosities on the boundary friction of articular cartilage. Journal of Tribology, ASME 1998;120:241-251.

Basser PJ, Schneiderman R, Bank RA, Wachtel E, Maroudas A. Mechanical properties of the collagen network in human articular cartilage as measured by osmotic stress technique. Arch Biochem Biophys 1998;351:207-19.

Bathe M, Rutledge G, Grodzinsky A, Tidor B. A Course-Grained Molecular Model for Glycosaminoglycans: Application to Chondroitin Sulfate, and Hyaluronic Acid. Biophysical Journal. 2005;88:3870-3887.

Berendsen, H.J.C., Postma, J.P.M., van Gunsteren, W.F., and Hermans, J., 1981, Interaction Model for Water in Relation to Protein Hydration, Reidel Publishing Company Dordrecht, Dordrecht, pp. 331-342.

Brooks BR, Bruccoleri RE, Olafson BD, States DJ, Swaminathan S, Karplus M. "CHARMM: A program for macromolecular energy, minimization, and dynamics calculations". J Comp Chem 1983;4: 187-217.

Buckwalter J, Mankin H. Articular cartilage repair and transplantation. Arthritis \& Rheumatism. 1998;41(8):1331-1342.

Buschmann, M. D. and Grodzinsky, A. J. A molecular model of proteoglycan-associated electrostatic forces in cartilage mechanics. J. Biomech. Eng. 1995;117, 179-192.

Ercolessi, F. A Molecular Dynamics Primer, Spring College in Computational Physics ICTP, Trieste, 1997.

Garemyr, R., and A. Elofsson. Study of the electrostatic treatment in molecular dynamics simulations. Proteins Struct. Funct. Genet. 1999;37:417-428.

Gibbs, J. (1906) The Scientific Papers of J. Willard Gibbs, Vol. 1, Thermodynamics, (London: Longmans). Reprinted by (Woodbridge, Connecticut: Ox Bow Press, 1993). 
Hamerman D, Schubert M. Diarthrodial joints, an essay. Am J Med. 1962;33:555-90.

Heinegard D, Goldberg A. Structure and biology of cartilage and bone noncollagenous macromolecules. FASEB J 1989;3:2042-51.

Heinegard, D., and Y. Sommarin, Proteoglycans: an overview. Methods Enzymol. 1987;144:305-372.

Hodge, W. A., Fijan, R. S., Carlson, K. L., Burgess, R. G., Harris, W. H. and Mann, R. W. Contact pressures in the human hip joint measured in vivo. Proc. Nat. Acad. Sci. USA 1986;83:2879-2883.

IUPAC (International Union of Pure and Applied Chemistry) Compendium of Chemical Terminology, 2nd Edition, 1997.

Lethbridge-Cejku M, Schiller J, Bernadel L. Summary health statistics for US adults: National Health Interview Survey, 2006. Vital Health Stat 10. 2006;235:1-153.

Marcus, R. A., "Calculation of Thermodynamic Properties of Polyelectrolytes," 7. Chem. Phys. 1955, Vol. 23, pp. 1057-1068.

Maroudas, A., "Physico-chemical Properties of Articular Cartilage," (Freeman, M. A. R., ed.) Adult Articular Cartilage 1979, 2nd ed., pp. 215-290, Pitman, Tunbridge Wells, England.

Mourao PAS. Distribution of chondroitin 4-sulfate and chondroitin 6-sulfate in human articular and growth cartilage. Arthritis Rheum 1988;31:1028-33.

Mow VC, Rosenwasser MP. Articular cartilage: biomechanics. In: Woo SLY, Buckwalter JA, eds. Injury and Repair of the Musculoskeletal Soft Tissues. Park Ridge, Ill: American Academy of Orthopaedic Surgeons; 1988:427-463.

Ng L, Grodzinsky AJ, Patwari P, Sandy J, Plaas A, Ortiz C. Individual cartilage aggrecan macromolecules and their constituent glycosaminoglycans visualized via atomic force microscopy. J Struct Biol. 2003;143:242-57.

Rosenberg, S., "The Method of Sorting in Multivariate Research with Applications Selected from Cognitive Psychology and Person Perception," in Multivariate Applications in the Social Sciences, Eds., N. Hirschberg and L.G. Humphreys, Hillsdale, NJ: Erlbaum, 1982;117-142.

Roughley, P.J., 2006. The structure and function of cartilage proteoglycans. Eur. Cell Mater., 30(12):92-101.

Roux B, Simonson T: Implicit solvent models. Biophys Chem 1999;78:1-20.

Sandell L J. Articular cartilage and changes in arthritis An Introduction: Cell biology of osteoarthritis. Arthritis Research. 2001;3:107-113.

Shelley, J.C. Shelley, M.Y. Reeder, R.C. Bandyopadhyay, S. Klein, M.L., A coarse grain model for phospholipid simulations, J. Phys. Chem., B 2001;105:4464-4470.

Smith G, Knutsen G, Richardson J. A clinical review of cartilage repair techniques. Journal of Bone \& Joint Surgery, British Volume. 2005;87(4):445-449.

Solmajer T, Mehler EL. Electrostatic screening in molecular dynamics simulations. Protein Eng 1991;4:911-917. 
Stote, R., Dejaegere, A., Kuznetsov, D. and Falguet, L. (1999), "Theory of molecular dynamics simulations", Available at: http://www.ch.embnet.org/MD_tutorial/

Temenoff JS, Mikos AG. Review: tissue engineering for regeneration of articular cartilage. Biomaterials. 2000;21(5):431-40.

Tsiganos, C. P. Hardingham T. E. and Helen Muir, Aggregation of Cartilage Proteoglycans (Division of Biochemistry, Kennedy Institute of Rheumatology, London W6 7DW, U.K.).

Upholt WB, Olsen BR The active genes of cartilage. In: Hall BK, Newman SA (eds) Cartilage: molecular aspects 1991. CRC Press, Boca Raton, pp 1-57. 


\section{Appendix A}

\section{Derivation of Ionic Concentration}

The models used in the pulling simulations have 60 atoms of $\mathrm{Na}^{+}$in 12,898 to 26,576 molecules of water.

$$
\begin{gathered}
60 \_ \text {atoms } \mathrm{Na}^{+}\left(\frac{1 \mathrm{Mol}_{-} \mathrm{Na}^{+}}{6.022 \times 10^{23} \text { atoms } \mathrm{Na}^{+}}\right)=9.963 \times 10^{-23} \mathrm{Mol}_{-} \mathrm{Na}^{+} \\
12898 \text { molecules_ } \mathrm{H}_{2} \mathrm{O}\left(\frac{1 \mathrm{Mol}_{-} \mathrm{H}_{2} \mathrm{O}}{6.022 \times 10^{23} \text { molecules }_{-} \mathrm{H}_{2} \mathrm{O}}\right)=2.142 \times 10^{-20} \mathrm{Mol}_{-} \mathrm{H}_{2} \mathrm{O} \\
2.142 \times 10^{-20}{ }_{-} \mathrm{Mol}_{-} \mathrm{H}_{2} \mathrm{O}\left(\frac{18.01528 \mathrm{~g}}{1 \mathrm{Mol}_{-} \mathrm{H}_{2} \mathrm{O}}\right)\left(\frac{1 \mathrm{ml}}{1 \mathrm{~g}}\right)\left(\frac{1 \mathrm{Liter}}{1000 \mathrm{ml}}\right)=3.858 \times 10^{-22}{ }_{-} \mathrm{L}_{-} \mathrm{H}_{2} \mathrm{O}
\end{gathered}
$$

The definition of a molar solution is how many moles of solute are contained in one liter of solution.

$$
\left(\frac{9.963 \times 10^{-23}{ }_{-} \mathrm{Mol}_{-} \mathrm{Na}^{+}}{3.858 \times 10^{-22} \mathrm{~L}_{-} \mathrm{H}_{2} \mathrm{O}}\right)=0.258 \mathrm{M}_{-} \mathrm{Na}^{+}
$$


Repeating this derivation for 26576 water molecules produces the results shown in Table A.1.

Table A.1: Ionic concentrations for the smallest and largest systems used in the pulling simulations

\begin{tabular}{|c|c|c|c|}
\hline Spacing & Na+ atoms & H2O molecules & Ionic Concentration (M Na+) \\
\hline $1 \mathrm{~nm}$ & 60 & 12898 & 0.25 \\
\hline $4 \mathrm{~nm}$ & 60 & 26576 & 0.13 \\
\hline
\end{tabular}




\section{Appendix B}

\section{Sample Simulation Times}

Table B.1: This table shows simulation times for one sample for each simulation run.

\begin{tabular}{|l|c|c|c|c|c|}
\hline & $\begin{array}{c}\# \\
\text { Atoms }\end{array}$ & $\begin{array}{c}\text { Simulation } \\
\text { Time (ps) }\end{array}$ & \# Steps & $\begin{array}{c}\text { Real } \\
\text { Time } \\
\text { (hrs) }\end{array}$ & $\begin{array}{c}\# \\
\text { CPU's }\end{array}$ \\
\hline Single Chain (20 units) & 12661 & 2000 & 1000000 & 14.7 & 2 \\
\hline 5 Chain (2 nm spacing) & 41984 & 200 & 100000 & 22.1 & 2 \\
\hline $\begin{array}{l}\text { Pulling (1.5 nm } \\
\text { spacing) }\end{array}$ & 38709 & 1500 & 1500000 & 68.3 & 2 \\
\hline
\end{tabular}




\section{Appendix C}

\section{Steps to Run Simulation}

Below are the general steps for running a simulation with Gromacs. Please refer to the manual for more information on file types. Help files can be obtained by typing the command then $-h$ (i.e. $g_{-}$energy $-h$ ). Some commands don't have help files but some will have manuals. To read the manual type man then the command (i.e. man $\mathrm{rm}$ )

1. Obtain a .pdb file of the molecule (These can be created or found using chemistry websites)

2. Convert .pdb file to a .gro structure file.

ex. pdb2gmx $-f$ molecule.pdb $-p$ topol.top -o molecule.gro 
3. Select force field

There are now 4 files

molecule.pdb (original pdb structure file)

molecule.gro (gromacs structure file)

topol.top (generated topology file)

posre.itp (position restraint file)

4. Write or copy a .mdp file that contains the parameters for the simulation.

5. Create an index file (.ndx) to group atoms into categories.

ex. make_ndx $-f$ molecule.gro $-o$ index. $n d x$

6. Run grompp to pre-compile

ex. grompp -fem_param.mdp -c molecule.gro $-p$ topol.top

-o em_topol.tpr - po $-n$ index.ndx

These are the current files

molecule.pdb

molecule.gro

topol.top

posre.itp

em_param.mdp

index.ndx

mdout.mdp (complete parameter file output from grompp)

em_topol.tpr (binary topology file output from grompp)

7. Run energy minimization

ex. mdrun $-v-$ nice $O-s$ topol.tpr $-o$ em_traj.trr $-c$ em_out.gro

-e em_energy.edr 
New files

$\begin{array}{ll}\text { md.log } & \text { (log of simulations) } \\ \text { em_energy.edr } & \text { (binary file containing energy data) } \\ \text { em_out.gro } & \text { (structure of last conformation) } \\ \text { em_traj.trr } & \text { (trajectory data) }\end{array}$

8. Create a periodic boundary box

ex. editconf $-f$ em_out.gro -o box.gro $-d 0.75-b t$ cubic

9. Fill the box with water

ex. genbox -cp box.gro -o water.gro - p topol.top -cs

10. Pre compile input files

ex. grompp $-f m d \_$param.mdp $-c$ water.gro $-p$ topol.top $-o$ md_topol.tpr

11. Run molecular dynamics simulation

ex. mdrun -nice $O-V-S$ md_topol.tpr $-o$ md_traj.trr $-c$ md_out.gro

-e md_energy.edr 


\section{Appendix D}

\section{Files}

Single Chain Simulation - 20 unit chain

Structure file sample

Topology file sample

Molecular dynamics parameter file

Five Chain Simulation - $2 \mathrm{~nm}$ spacing

Structure file sample

Topology file sample

Molecular dynamics parameter file

Pulling Simulation - $1.5 \mathrm{~nm}$ spacing

Structure file sample

Topology file sample

Molecular Dynamics parameter file

Pulling parameter file

Pulling output data file sample

Force Field Files

Atom mass and descriptions

Bonded interaction parameters

Non-bonded interaction parameters 


\section{Single Chain Simulation - 20 unit chain Structure file sample}

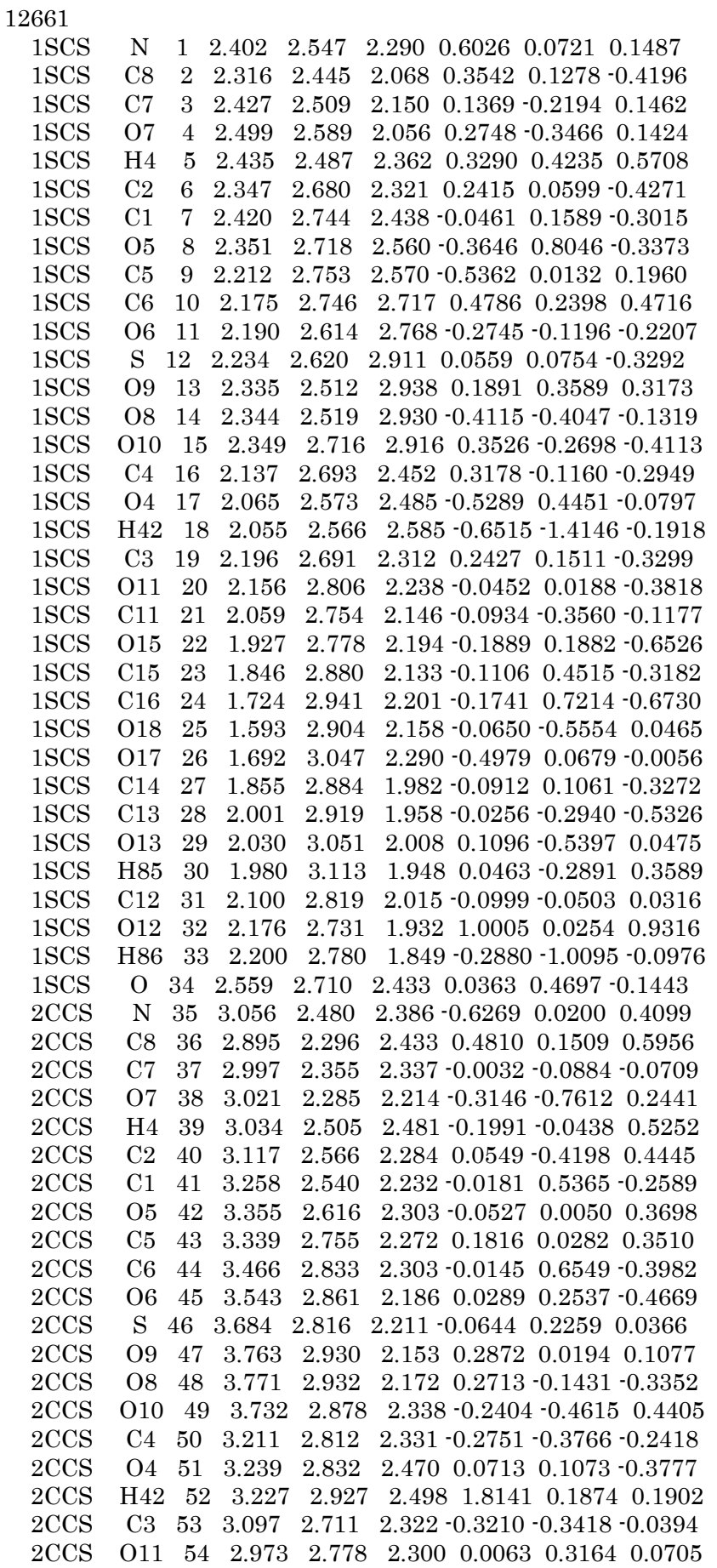




\section{Single Chain Simulation - 20 unit chain Topology file sample}

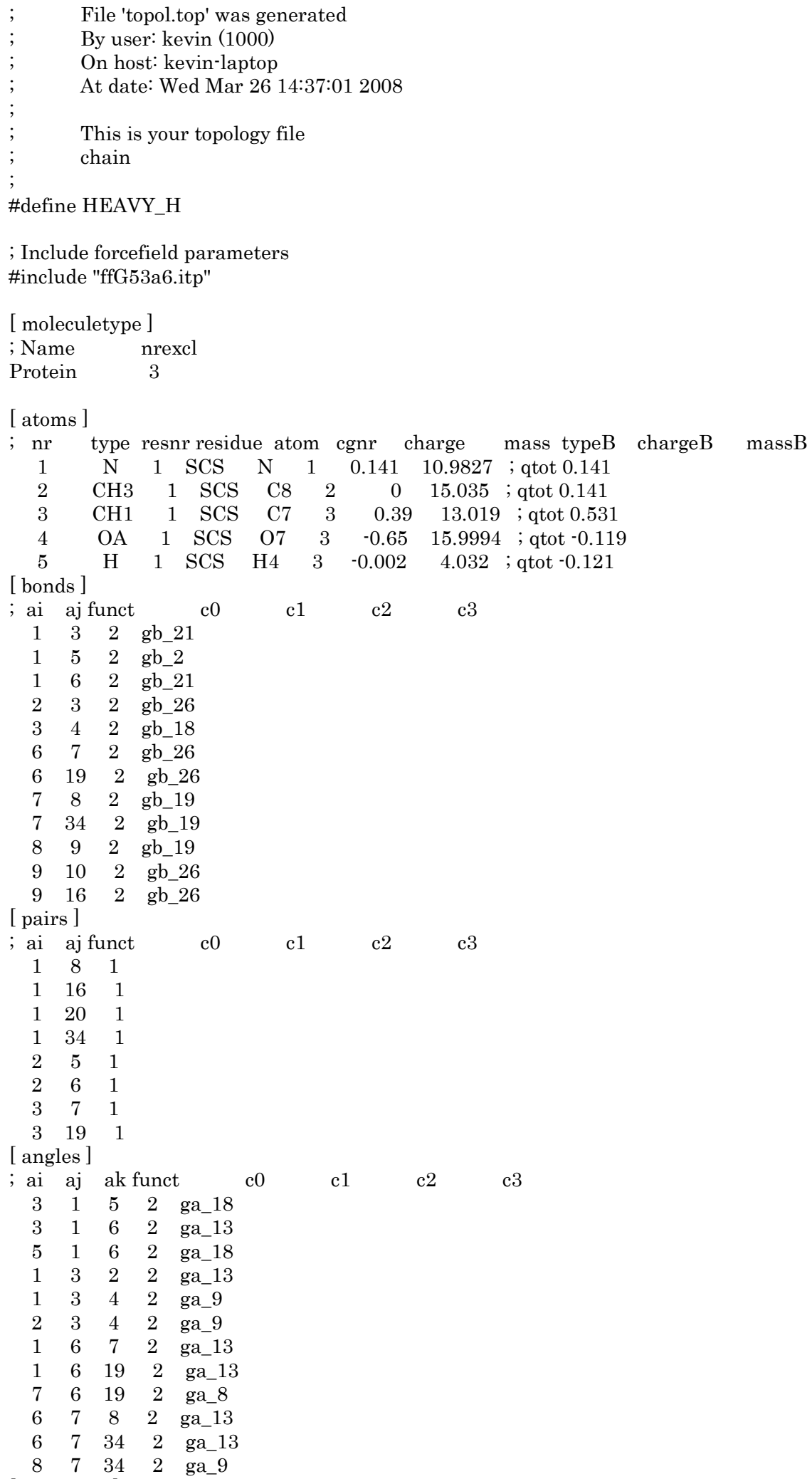




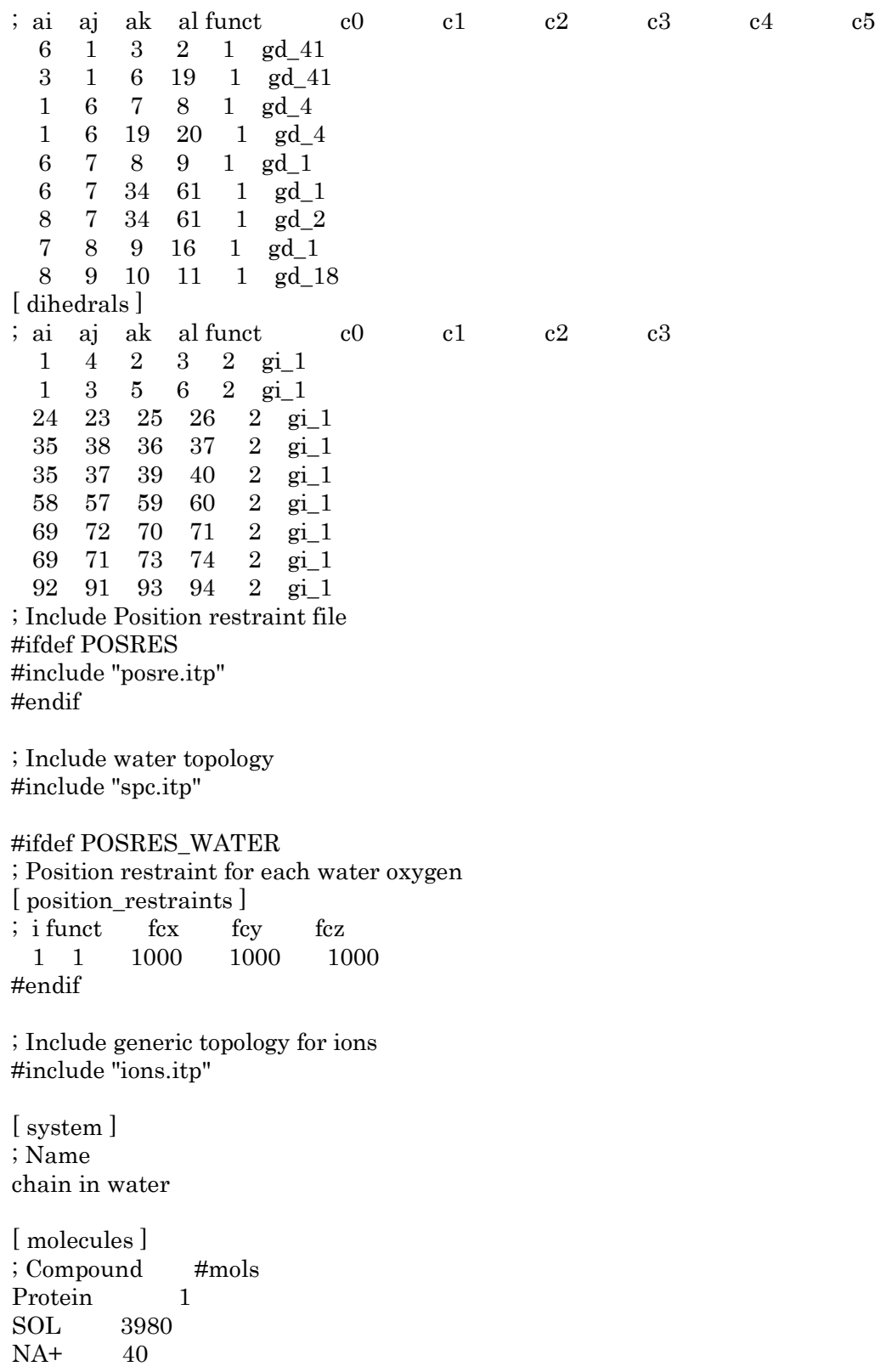




\section{Single Chain Simulation - 20 unit chain MD parameter file}

File 'out.mdp' was generated

By user: marco (1000)

On host: biogs26

At date: Fri Apr 4 03:51:00 2008

; VARIOUS PREPROCESSING OPTIONS

title =

; Preprocessor - specify a full path if necessary.

$\mathrm{cpp} \quad \mathrm{cpp}$

include $\quad=$

define $\quad=$

; RUN CONTROL PARAMETERS

integrator $=\mathrm{md}$

; Start time and timestep in ps

tinit $\quad=0$

$\mathrm{dt} \quad=0.002$

nsteps $\quad=1000000$

; For exact run continuation or redoing part of a run

init_step $\quad=0$

; mode for center of mass motion removal

comm-mode = Linear

; number of steps for center of mass motion removal

nstcomm $\quad=1$

; group(s) for center of mass motion removal

comm-grps = Aggrecan

; LANGEVIN DYNAMICS OPTIONS

; Friction coefficient (amu/ps) and random seed

bd-fric $\quad=0$

ld-seed $\quad=1993$

; ENERGY MINIMIZATION OPTIONS

; Force tolerance and initial step-size

emtol $=10$

emstep $\quad=0.001$

; Max number of iterations in relax_shells

niter $\quad=20$

; Step size $\left(\mathrm{ps}^{\wedge} 2\right)$ for minimization of flexible constraints

fcstep $\quad=0$

; Frequency of steepest descents steps when doing CG

nstcgsteep $\quad=1000$

nbfgscorr $\quad=10$

; OUTPUT CONTROL OPTIONS

; Output frequency for coords (x), velocities (v) and forces (f)

nstxout $\quad=1000$

nstvout $\quad=1000$

nstfout $\quad=0$

; Checkpointing helps you continue after crashes

nstcheckpoint $\quad=1000$

; Output frequency for energies to log file and energy file

nstlog $=1000$

nstenergy $\quad=100$

; Output frequency and precision for xtc file

nstxtcout $\quad=0$

xtc-precision $\quad=1000$

; This selects the subset of atoms for the xtc file. You can ; select multiple groups. By default all atoms will be written. xtc-grps 


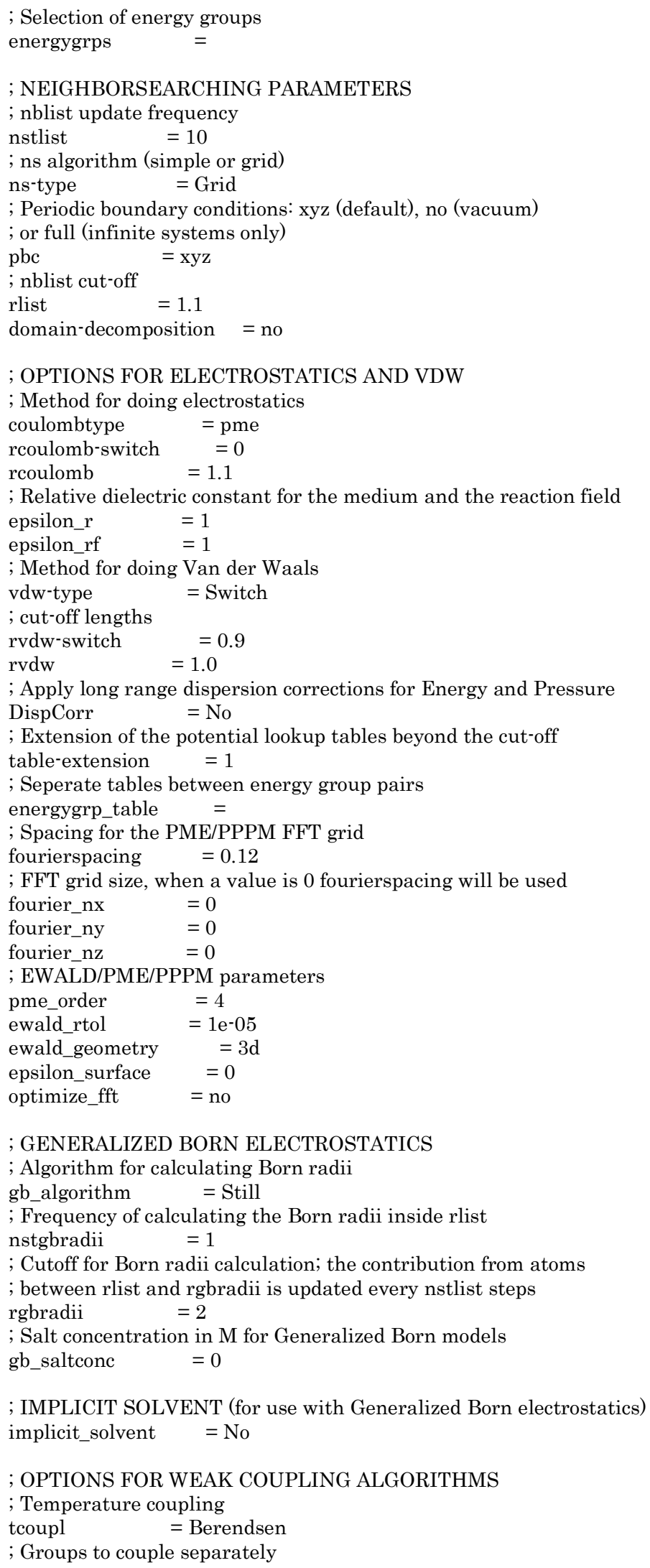




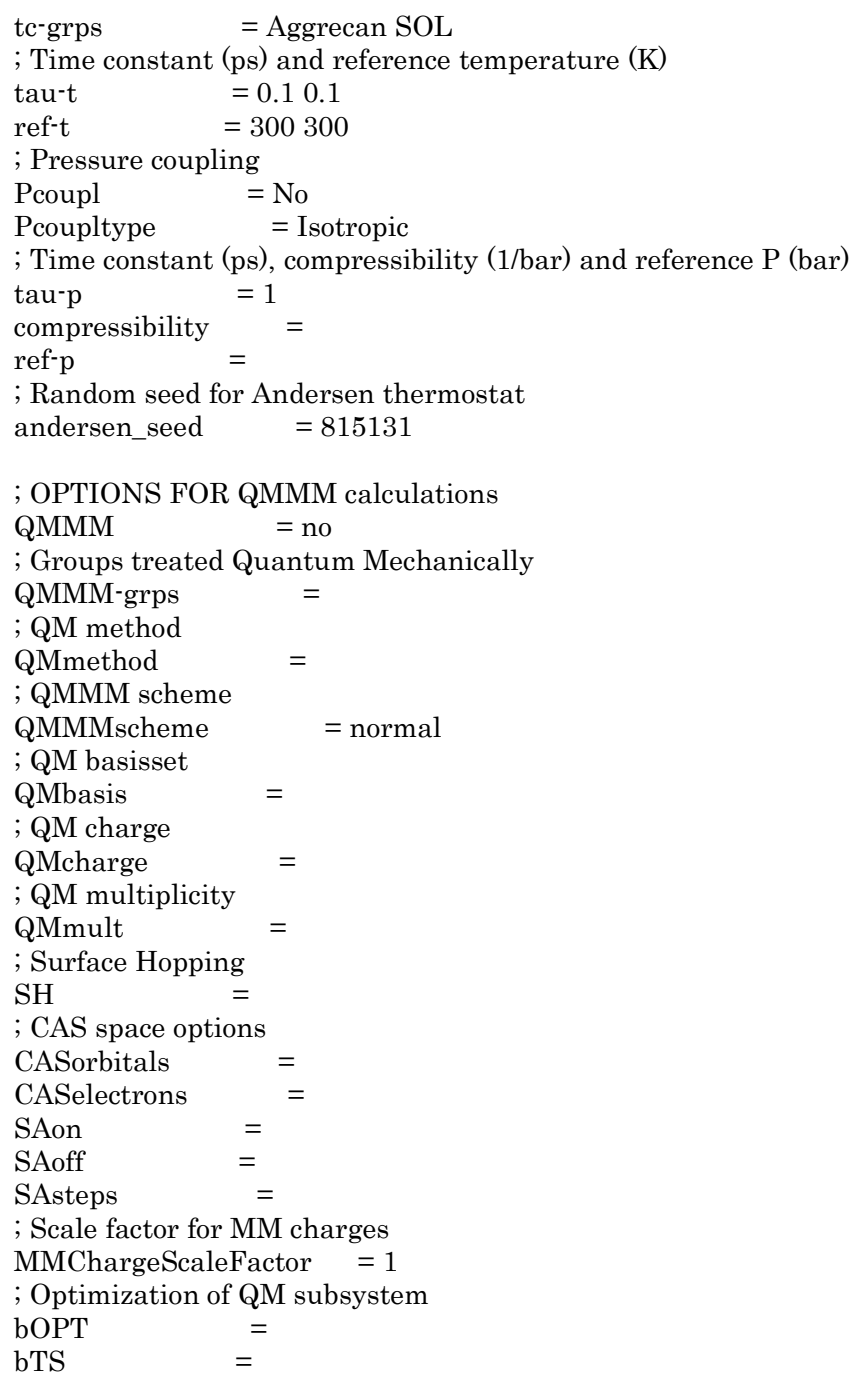




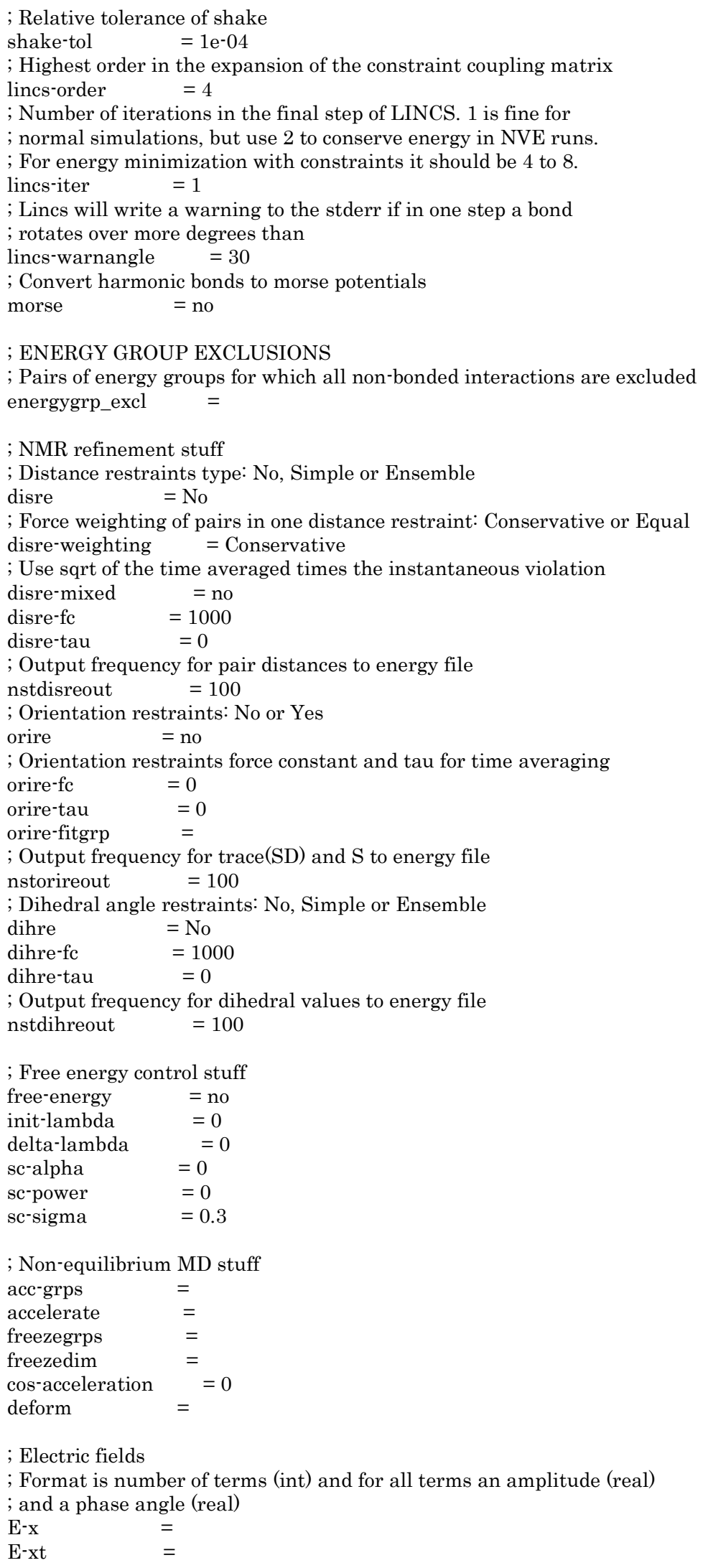

; Non-equilibrium MD stuff

acc-grps =

accelerate $\quad=$

freezegrps =

freezedim $\quad=$

cos-acceleration $\quad=0$

deform $\quad=$

; Electric fields

; Format is number of terms (int) and for all terms an amplitude (real)

; and a phase angle (real)

$\mathrm{E}-\mathrm{x} \quad=$

E-xt $\quad=$ 


$\begin{array}{ll}\text { E-y } & = \\ \text { E-yt } & = \\ \text { E-z } & = \\ \text { E-zt } & =\end{array}$

; User defined thingies user1-grps

$=$

user2-grps

userint1

userint2

userint3

userint4

userreal1

userreal2

userreal3

userreal4

$=$

$=0$

$=0$

$=0$

$=0$

$=0$

$=0$

$=0$
$=0$ 


\section{Five Chain Simulation - $2 \mathrm{~nm}$ spacing Structure file sample}

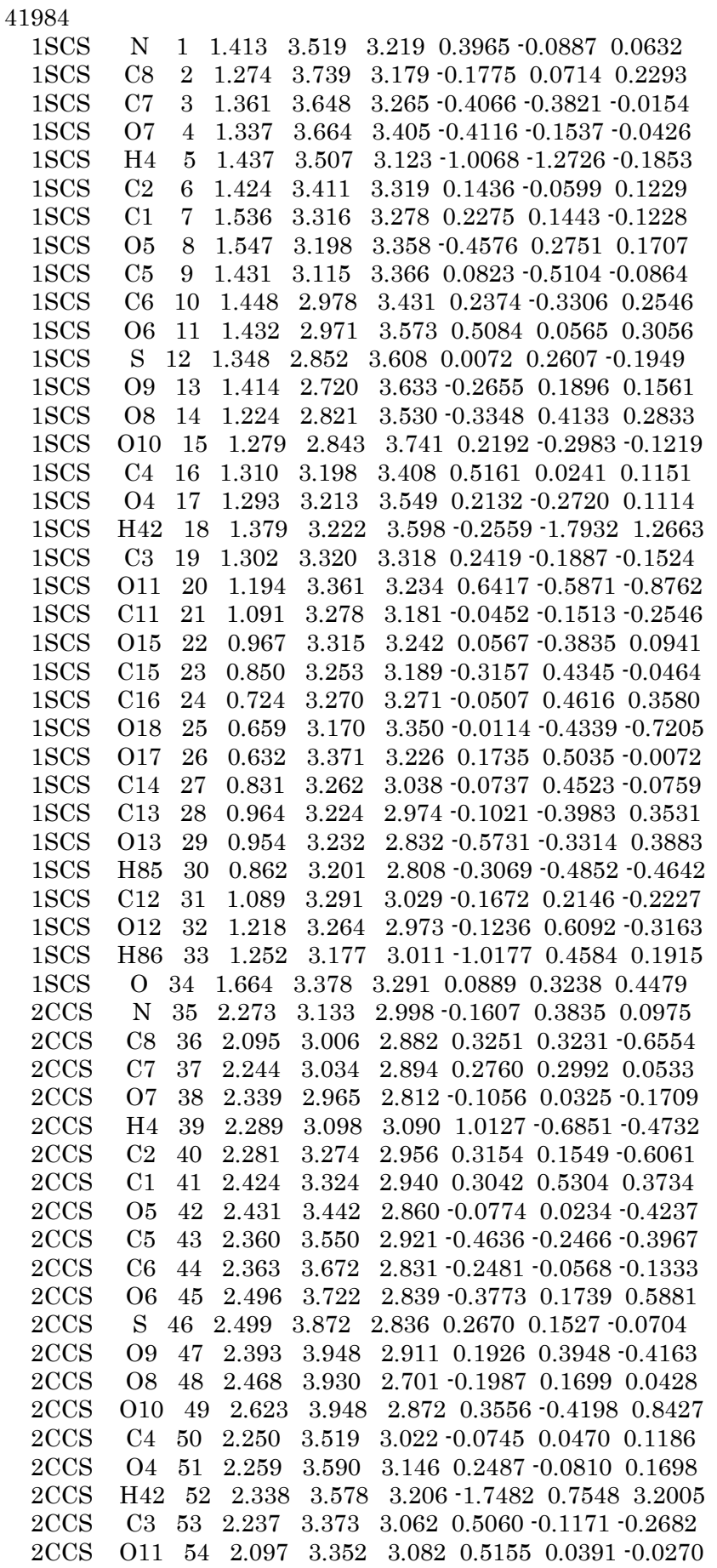


Five Chain Simulation - $2 \mathrm{~nm}$ spacing Topology file sample

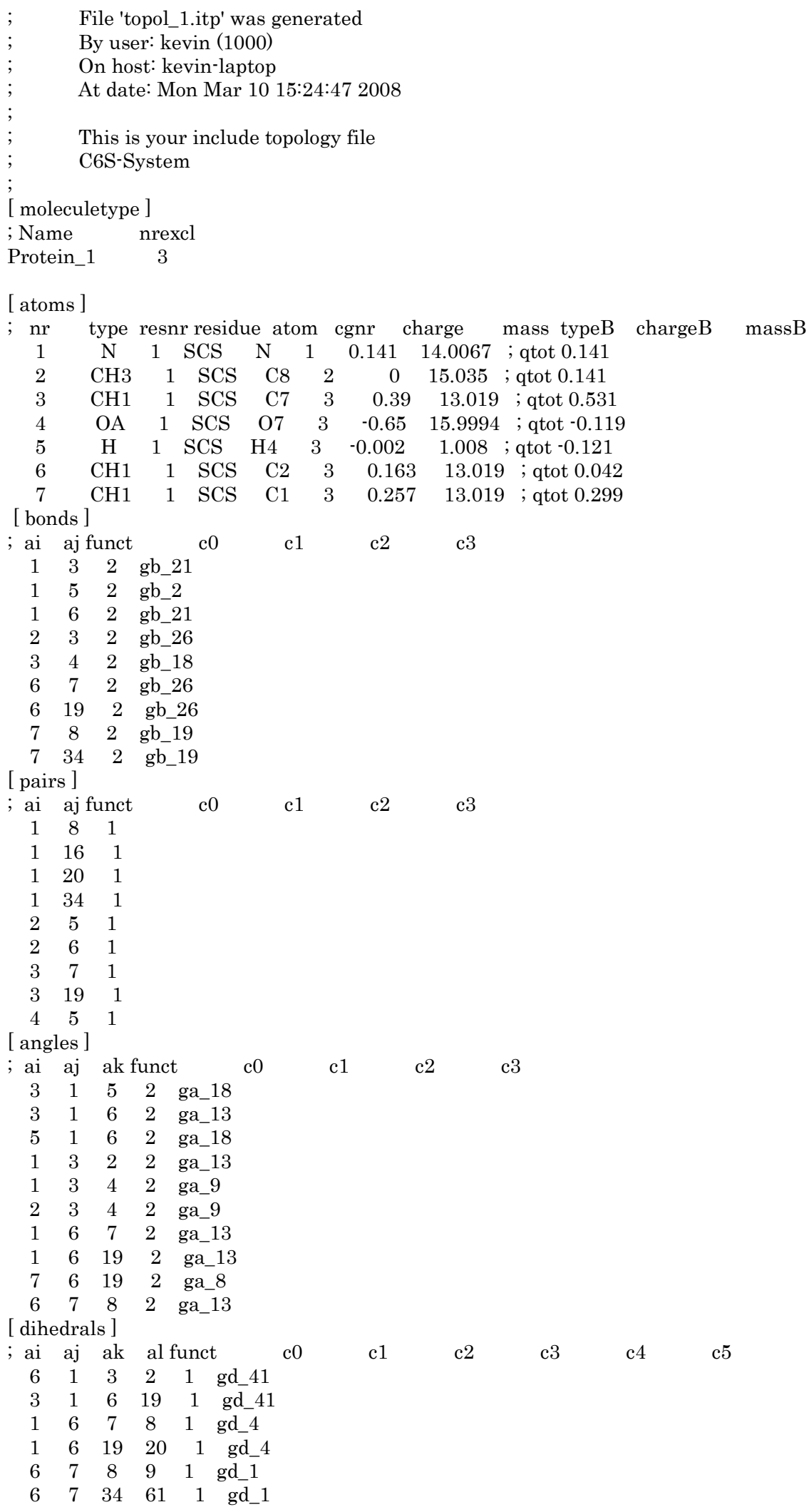


$\begin{array}{llllll}8 & 7 & 34 & 61 & 1 & \text { gd_2 }\end{array}$

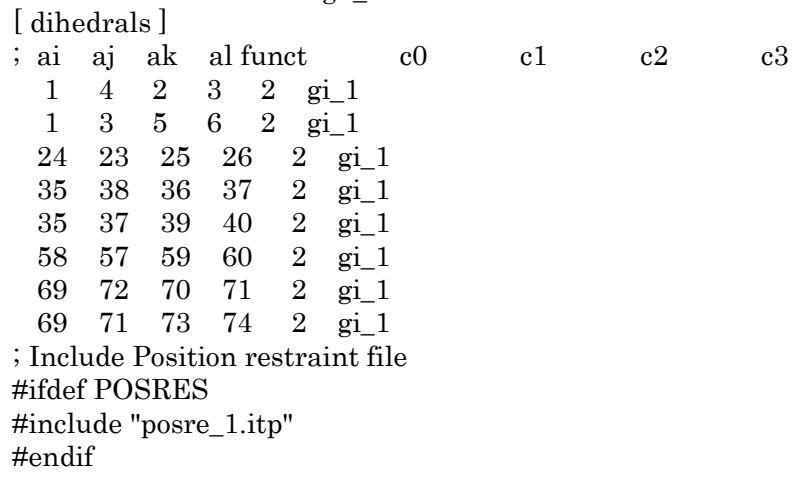




\section{Five Chain Simulation - $2 \mathrm{~nm}$ spacing MD parameter file}

File 'mdout.mdp' was generated

By user: kevin (1000)

On host: kevin-laptop

At date: Tue Jul 8 21:29:52 2008

; VARIOUS PREPROCESSING OPTIONS

title $=$

; Preprocessor - specify a full path if necessary.

$\mathrm{cpp} \quad=\mathrm{cpp}$

include $\quad=$

define $\quad=$

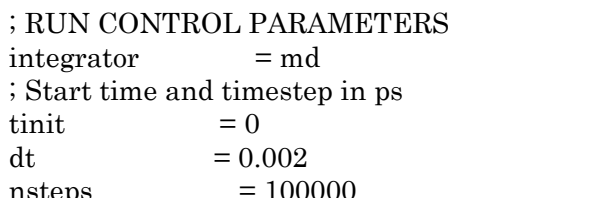

; For exact run continuation or redoing part of a run

init_step $\quad=0$

; mode for center of mass motion removal

comm-mode = Angular

; number of steps for center of mass motion removal

nstcomm $\quad=1$

; group(s) for center of mass motion removal

comm-grps = SCS

; LANGEVIN DYNAMICS OPTIONS

; Friction coefficient (amu/ps) and random seed

bd-fric $\quad=0$

ld-seed = 1993

; ENERGY MINIMIZATION OPTIONS

; Force tolerance and initial step-size

emtol $=10$

emstep $\quad=0.01$

; Max number of iterations in relax_shells

niter $\quad=20$

; Step size $\left(\mathrm{ps}^{\wedge} 2\right)$ for minimization of flexible constraints

fcstep $\quad=0$

; Frequency of steepest descents steps when doing CG

nstcgsteep $\quad=1000$

nbfgscorr $\quad=10$

; OUTPUT CONTROL OPTIONS

; Output frequency for coords (x), velocities (v) and forces (f)

nstxout $\quad=1000$

nstvout $\quad=1000$

nstfout $\quad=0$

; Checkpointing helps you continue after crashes

nstcheckpoint $\quad=1000$

; Output frequency for energies to log file and energy file

nstlog $\quad=1000$

nstenergy $\quad=100$

; Output frequency and precision for xtc file

nstxtcout $\quad=0$

xtc-precision $\quad=1000$

; This selects the subset of atoms for the xtc file. You can ; select multiple groups. By default all atoms will be written.

xtc-grps

$=$ 


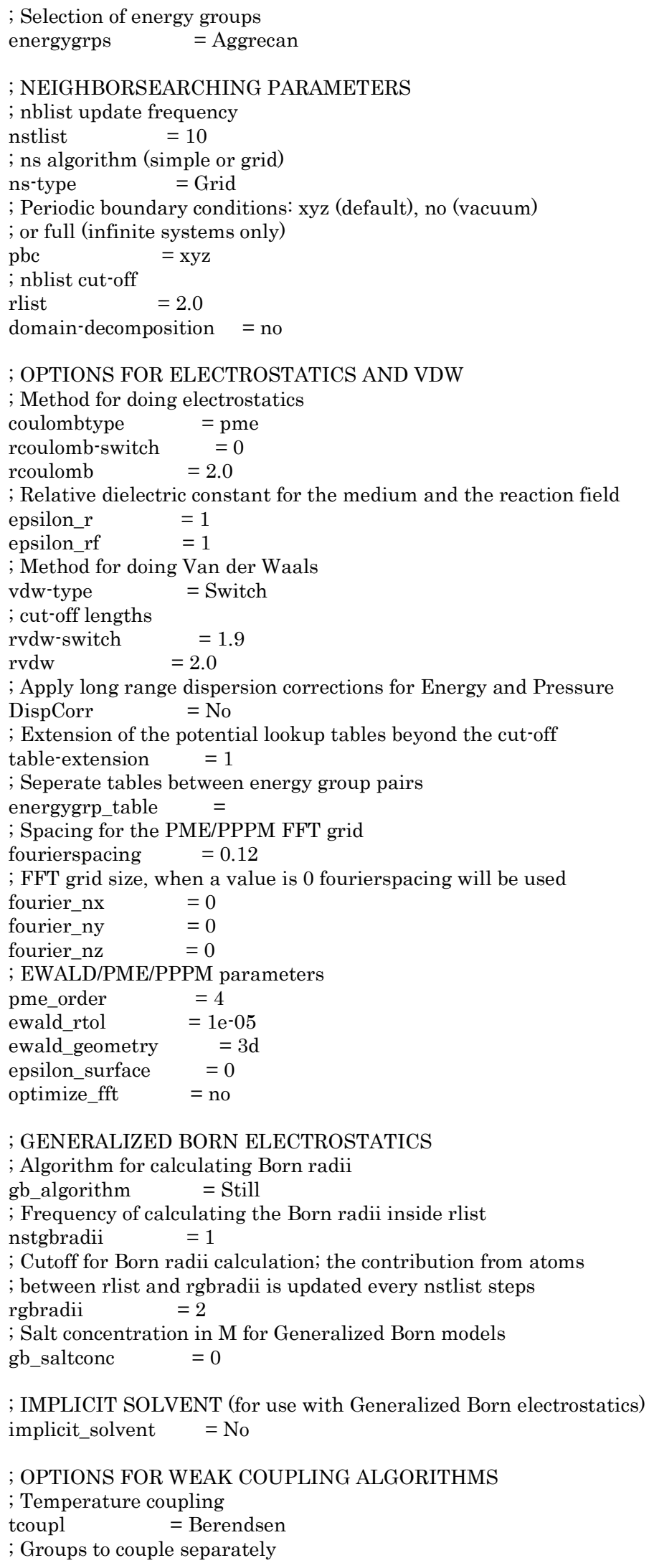

; IMPLICIT SOLVENT (for use with Generalized Born electrostatics) implicit_solvent $=$ No

; OPTIONS FOR WEAK COUPLING ALGORITHMS

; Temperature coupling 


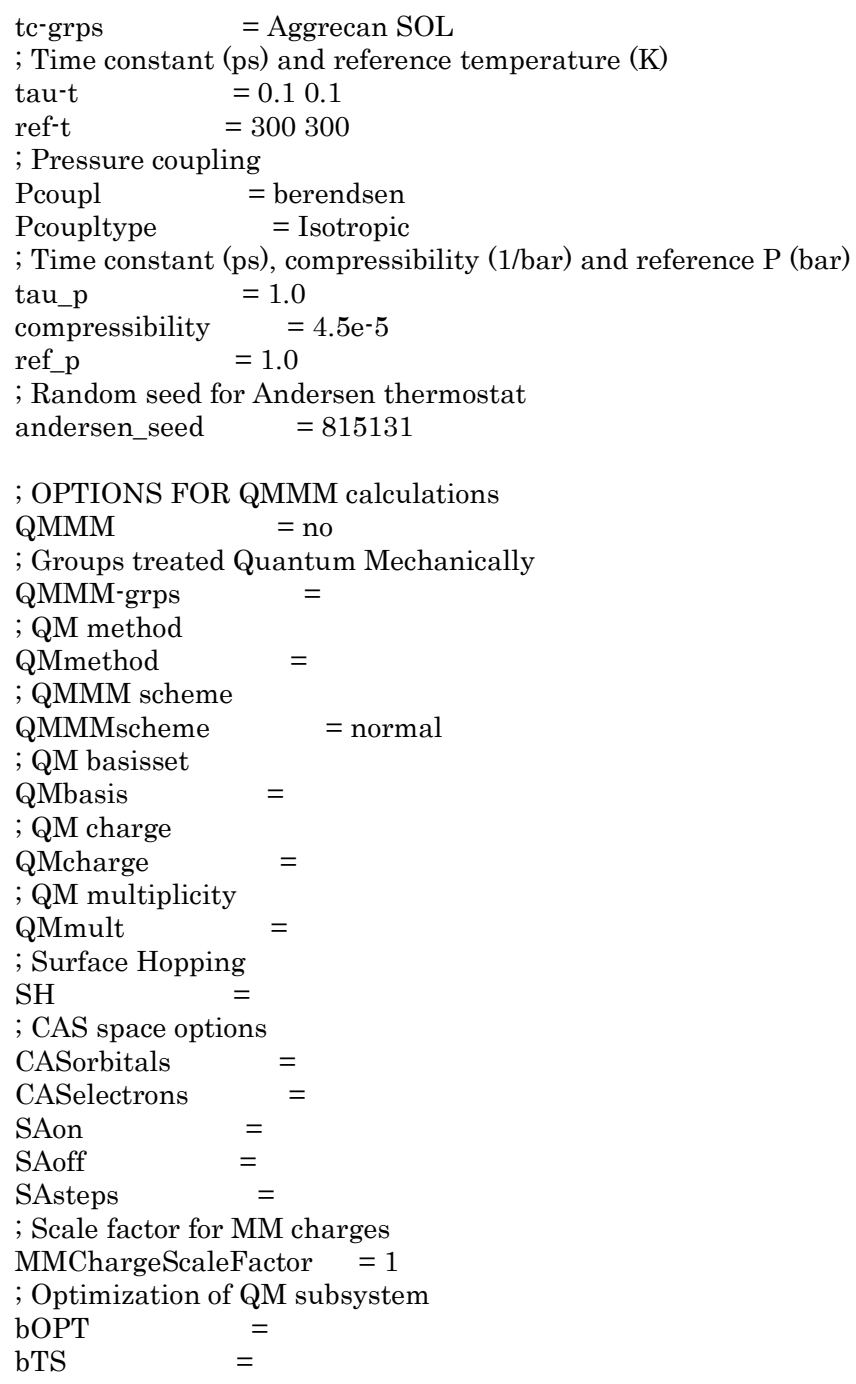




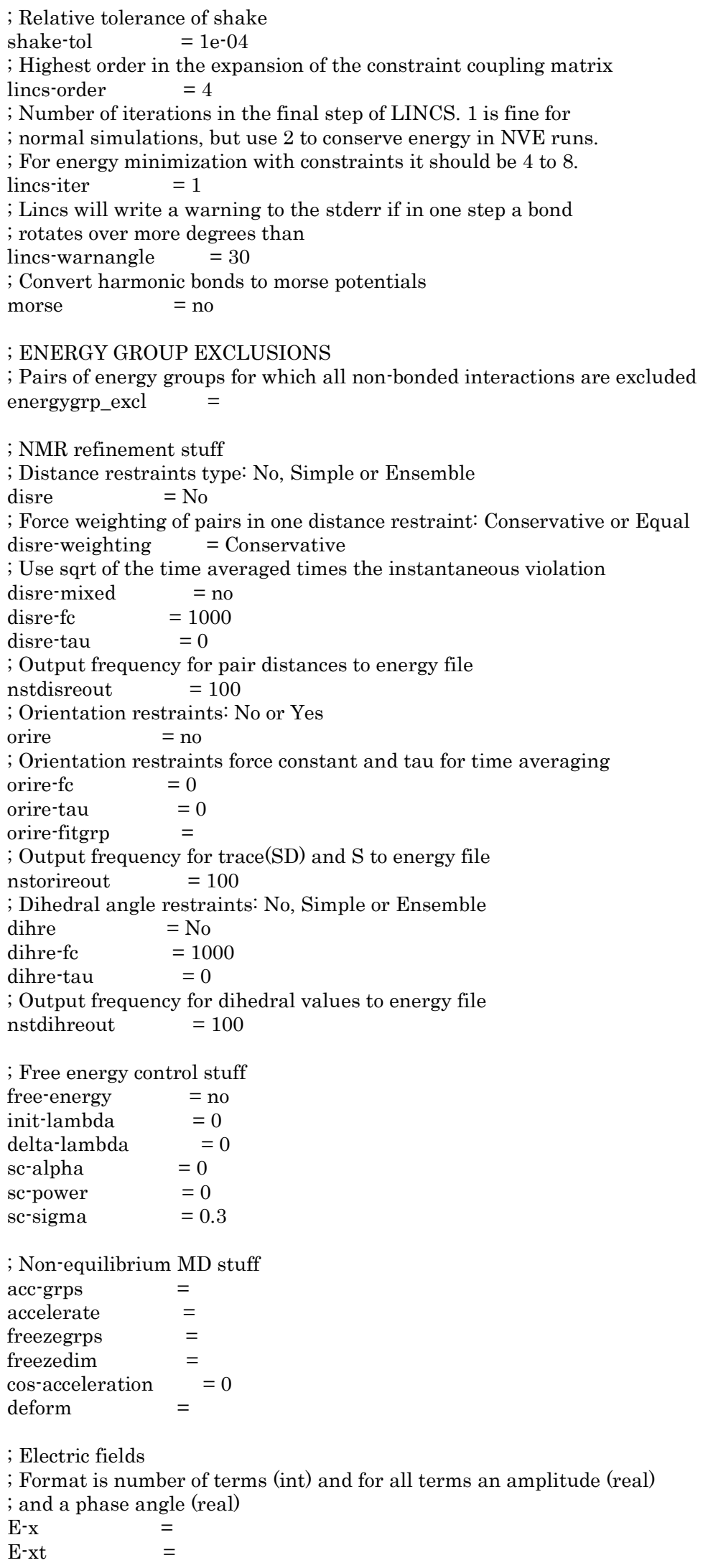

; Non-equilibrium MD stuff

acc-grps =

accelerate $\quad=$

freezegrps =

freezedim $\quad=$

cos-acceleration $\quad=0$

deform $\quad=$

; Electric fields

; Format is number of terms (int) and for all terms an amplitude (real)

; and a phase angle (real)

$\mathrm{E}-\mathrm{x} \quad=$

E-xt $\quad=$ 


$\begin{array}{ll}\text { E-y } & = \\ \text { E-yt } & = \\ \text { E-z } & = \\ \text { E-zt } & =\end{array}$

; User defined thingies user1-grps

$=$

user2-grps

userint1

userint2

userint3

userint4

userreal1

userreal2

userreal3

userreal4

$=$

$=0$

$=0$

$=0$

$=0$

$=0$

$=0$

$=0$
$=0$ 


\section{Pulling Simulation - 1.5 nm spacing Structure file sample}

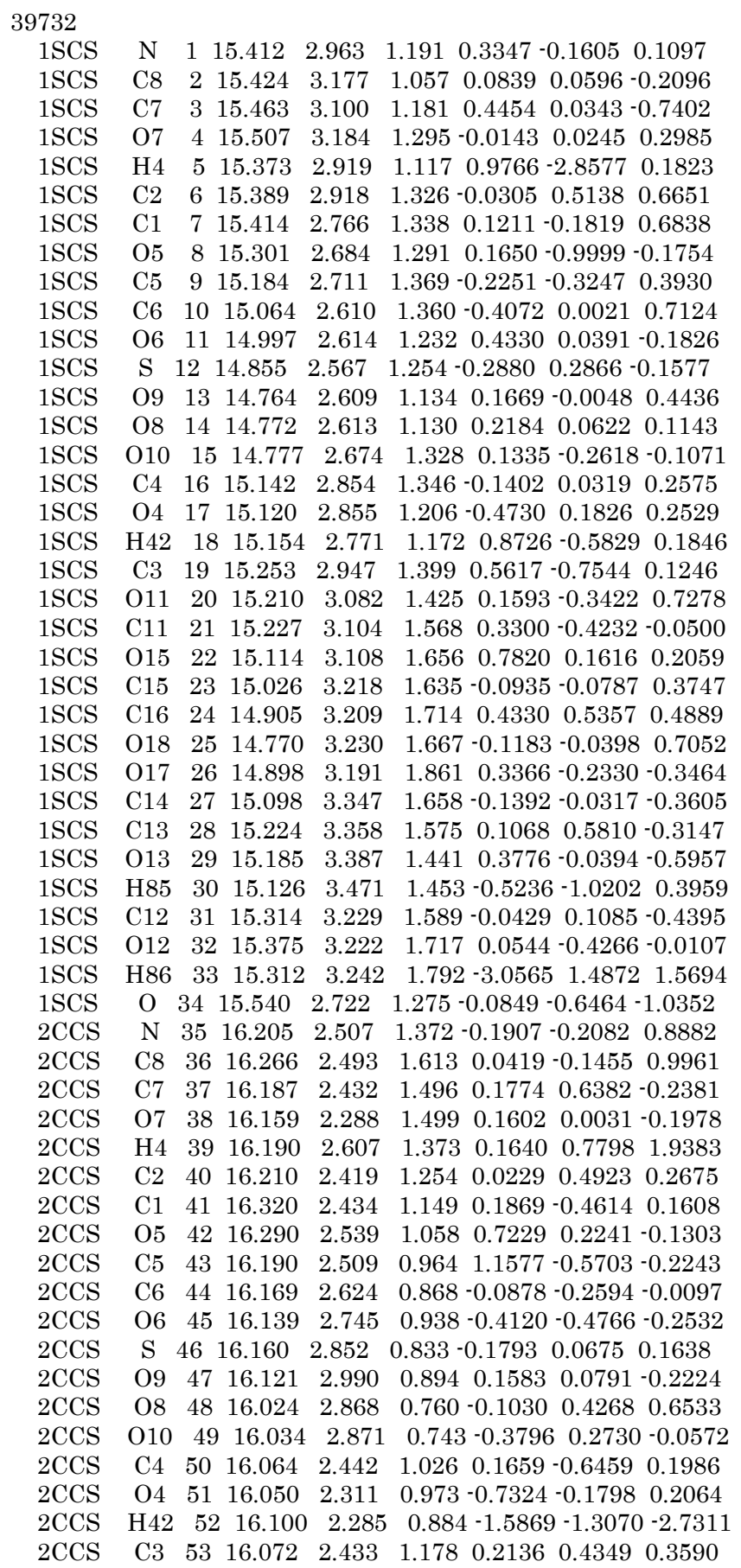




\section{Pulling Simulation - $1.5 \mathrm{~nm}$ spacing Topology file sample}

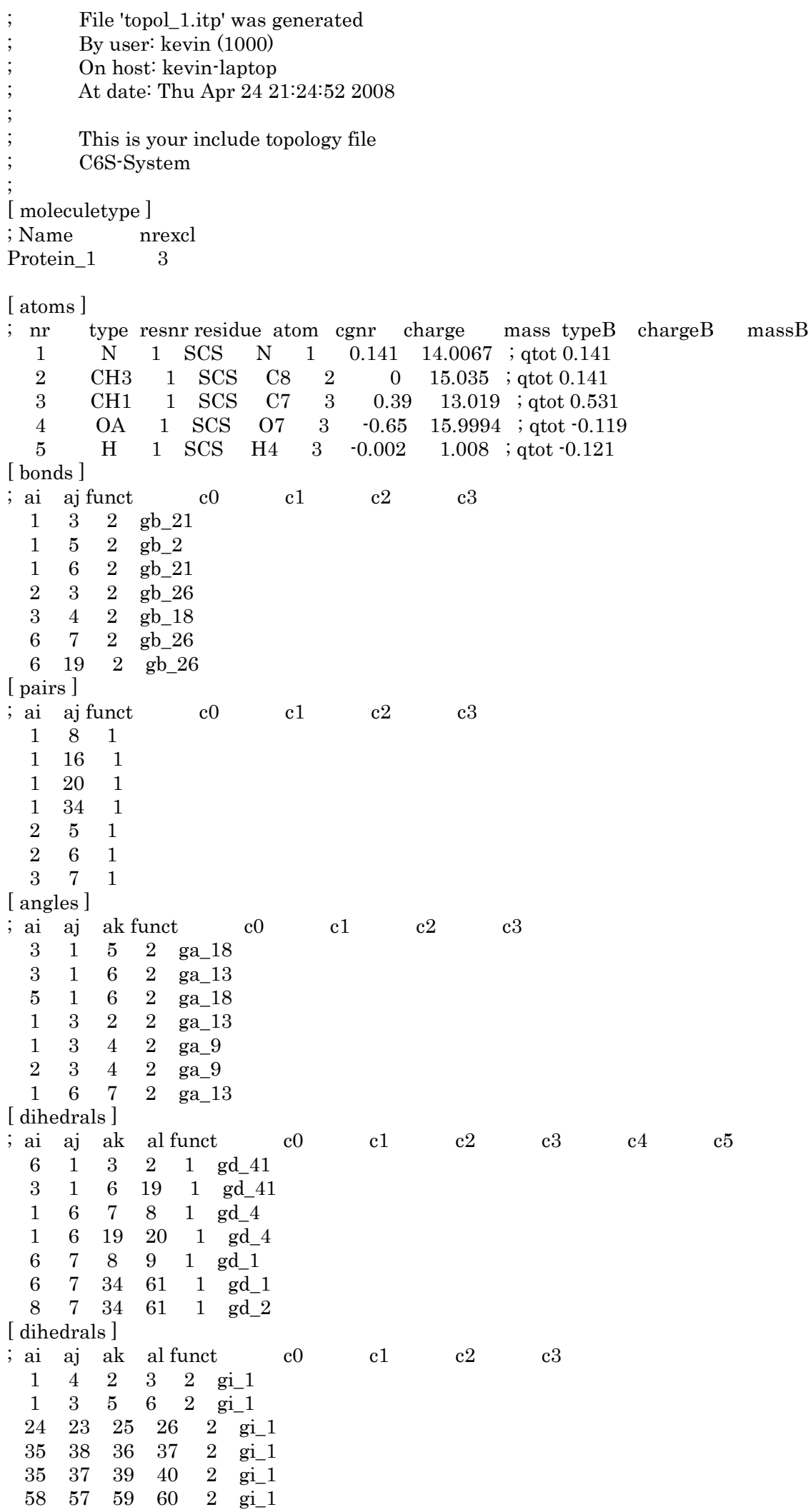


$\begin{array}{llllll}69 & 72 & 70 & 71 & 2 & \text { gi } 1\end{array}$

; Include Position restraint file \#ifdef POSRES

\#include "posre_1.itp"

\#endif 


\section{Pulling Simulation $-1.5 \mathrm{~nm}$ spacing MD parameter file}

File 'gromppout.mdp' was generated

By user: onbekend (0)

On host: onbekend

At date: Fri Mar 7 01:08:28 2008

; VARIOUS PREPROCESSING OPTIONS

title =

; Preprocessor - specify a full path if necessary.

$\mathrm{cpp} \quad=\mathrm{cpp}$

include $\quad=$

define $\quad=$

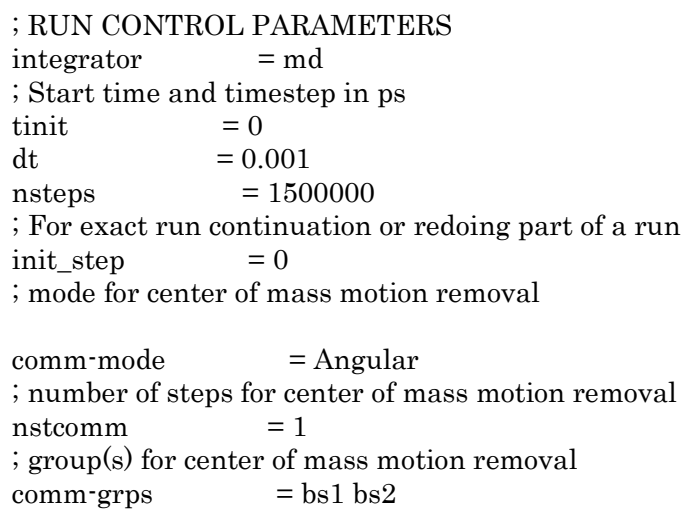

; LANGEVIN DYNAMICS OPTIONS

; Friction coefficient (amu/ps) and random seed

bd-fric $\quad=0$

ld-seed $\quad=1993$

; ENERGY MINIMIZATION OPTIONS

; Force tolerance and initial step-size

emtol $=10$

emstep $\quad=0.001$

; Max number of iterations in relax_shells

niter $\quad=20$

; Step size $\left(\mathrm{ps}^{\wedge} 2\right)$ for minimization of flexible constraints

fcstep $\quad=0$

; Frequency of steepest descents steps when doing CG

nstcgsteep $\quad=1000$

nbfgscorr $\quad=10$

; OUTPUT CONTROL OPTIONS

; Output frequency for coords (x), velocities (v) and forces (f)

nstxout $\quad=1000$

nstvout $\quad=1000$

nstfout $\quad=0$

; Checkpointing helps you continue after crashes

nstcheckpoint $\quad=1000$

; Output frequency for energies to log file and energy file

nstlog $\quad=1000$

nstenergy $\quad=100$

; Output frequency and precision for xtc file

nstxtcout $\quad=0$

xtc-precision $\quad=1000$

; This selects the subset of atoms for the xtc file. You can

; select multiple groups. By default all atoms will be written. 


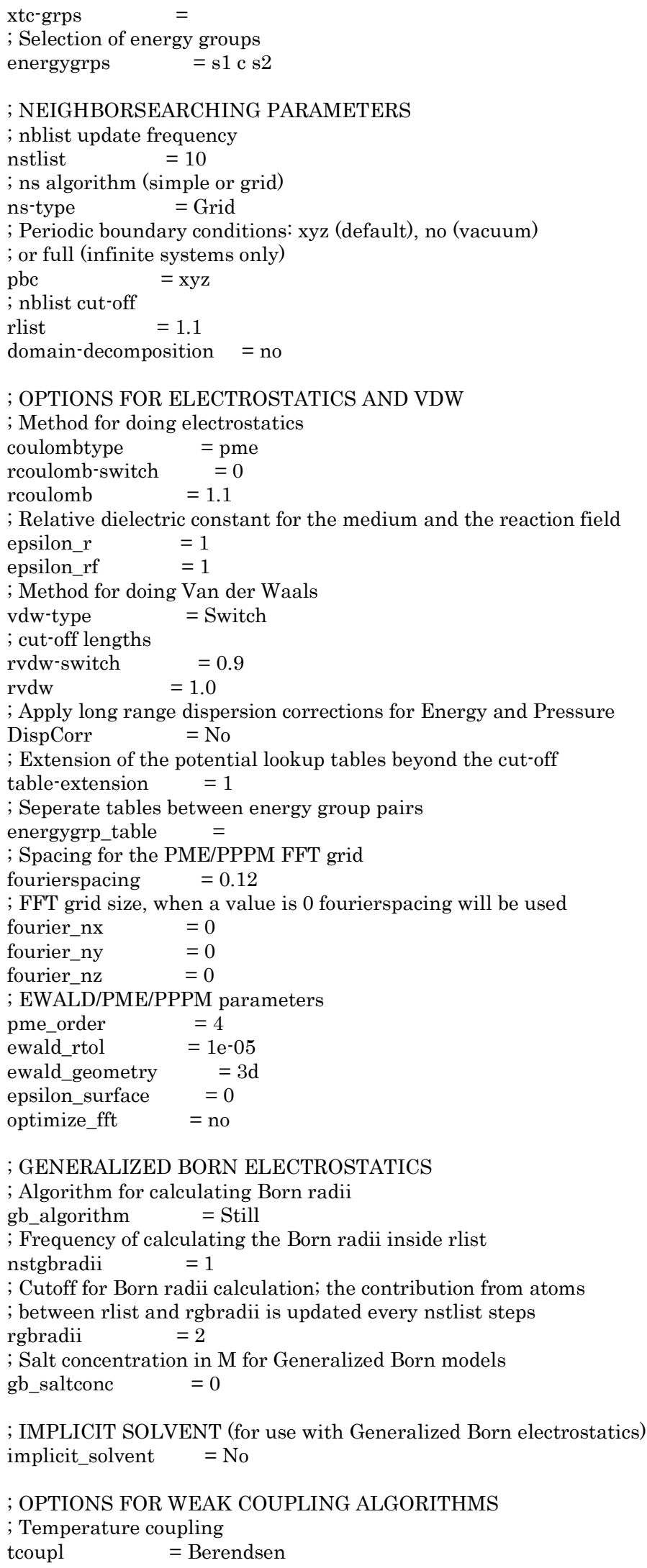

; GENERALIZED BORN ELECTROSTATICS

; Algorithm for calculating Born radii 


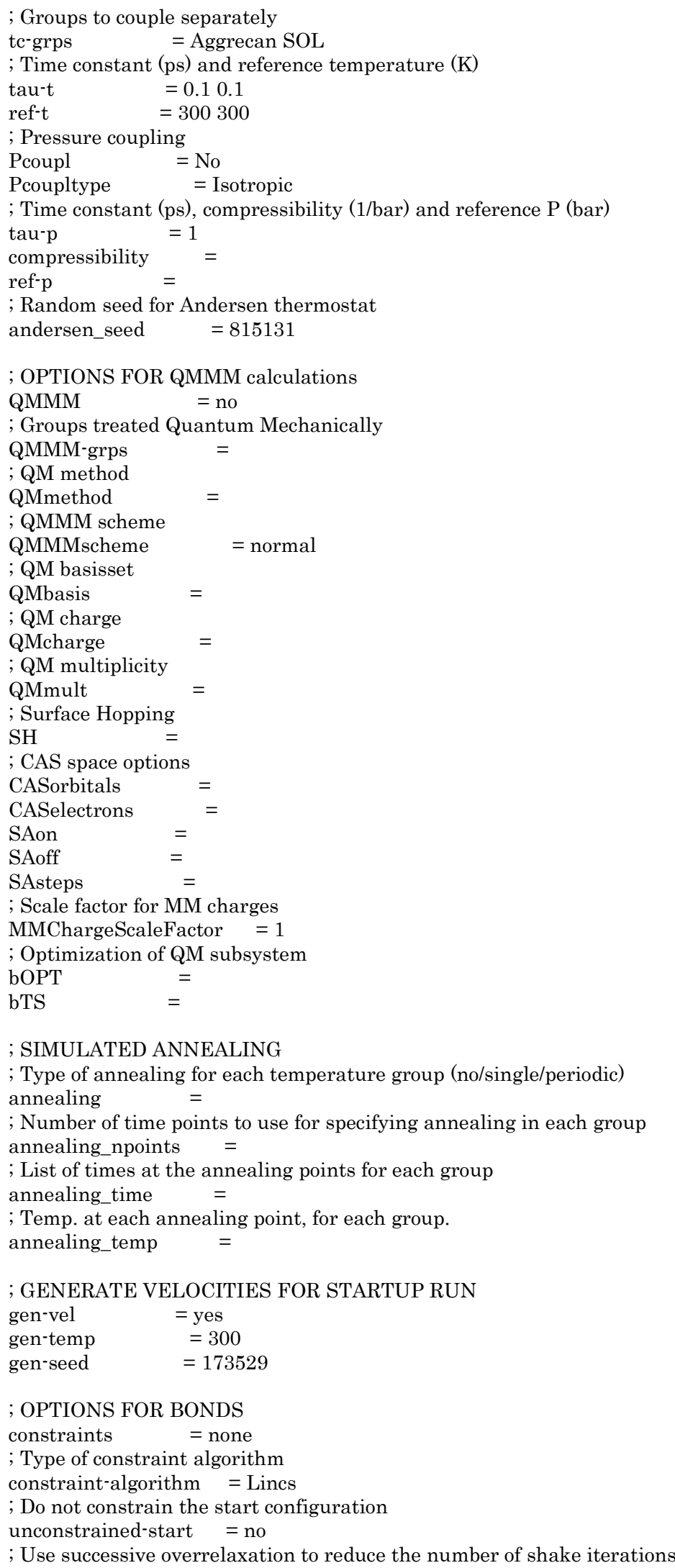




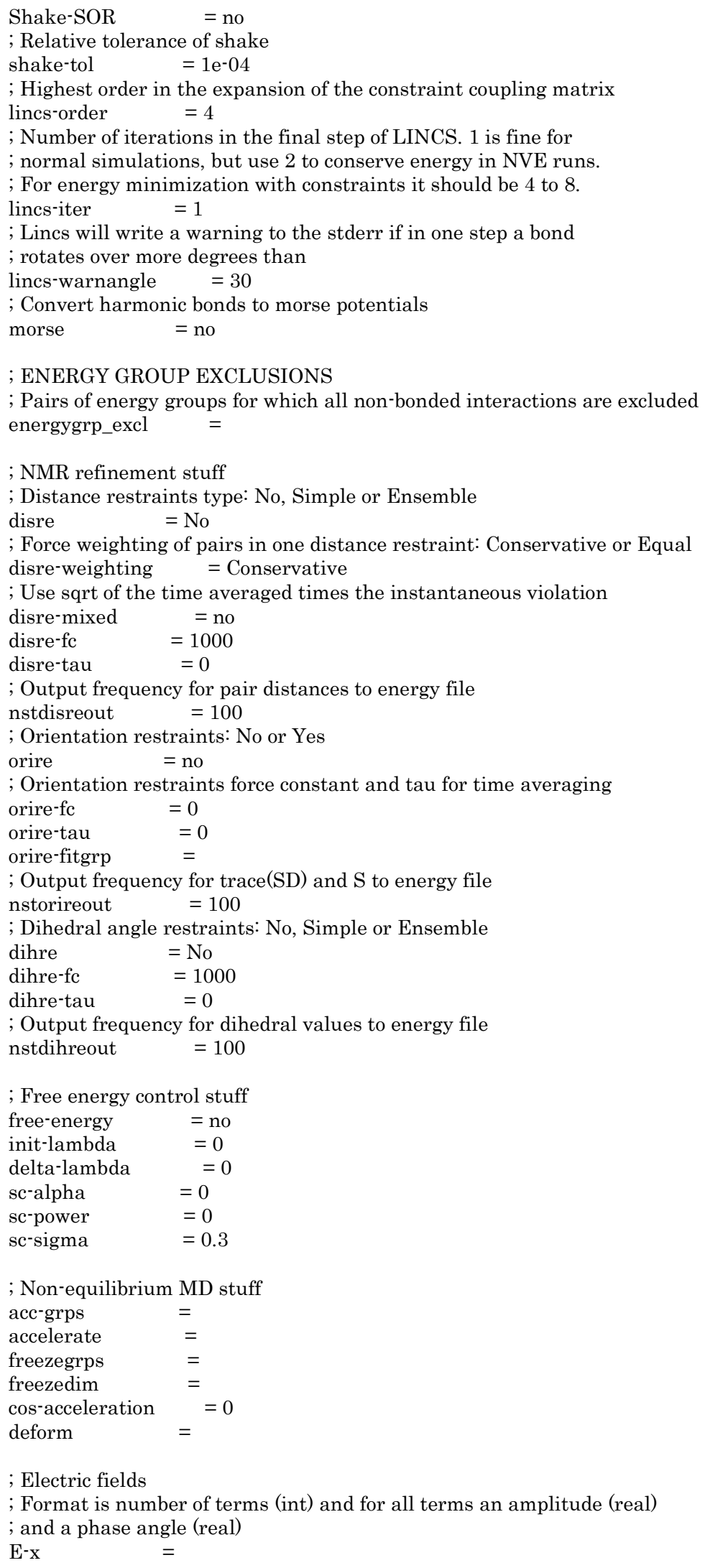

; Non-equilibrium MD stuff

acc-grps =

accelerate $\quad=$

freezegrps =

freezedim =

cos-acceleration $\quad=0$

deform =

; Electric fields

; Format is number of terms (int) and for all terms an amplitude (real)

; and a phase angle (real)

E-x 


$\begin{array}{ll}\text { E-xt } & = \\ \text { E-y } & = \\ \text { E-yt } & = \\ \text { E-z } & = \\ \text { E-zt } & =\end{array}$

; User defined thingies user1-grps = $\begin{array}{ll}\text { user2-grps } & = \\ \text { userint } 1 & =\end{array}$ userint2 $=0$

userint3 $\quad=0$

userint4 $=0$

userreal1 $=0$

userreal2 $=0$

userreal3 $=0$

userreal4 $=0$ 


\section{Pulling Simulation - $1.5 \mathrm{~nm}$ spacing Pulling parameter file}

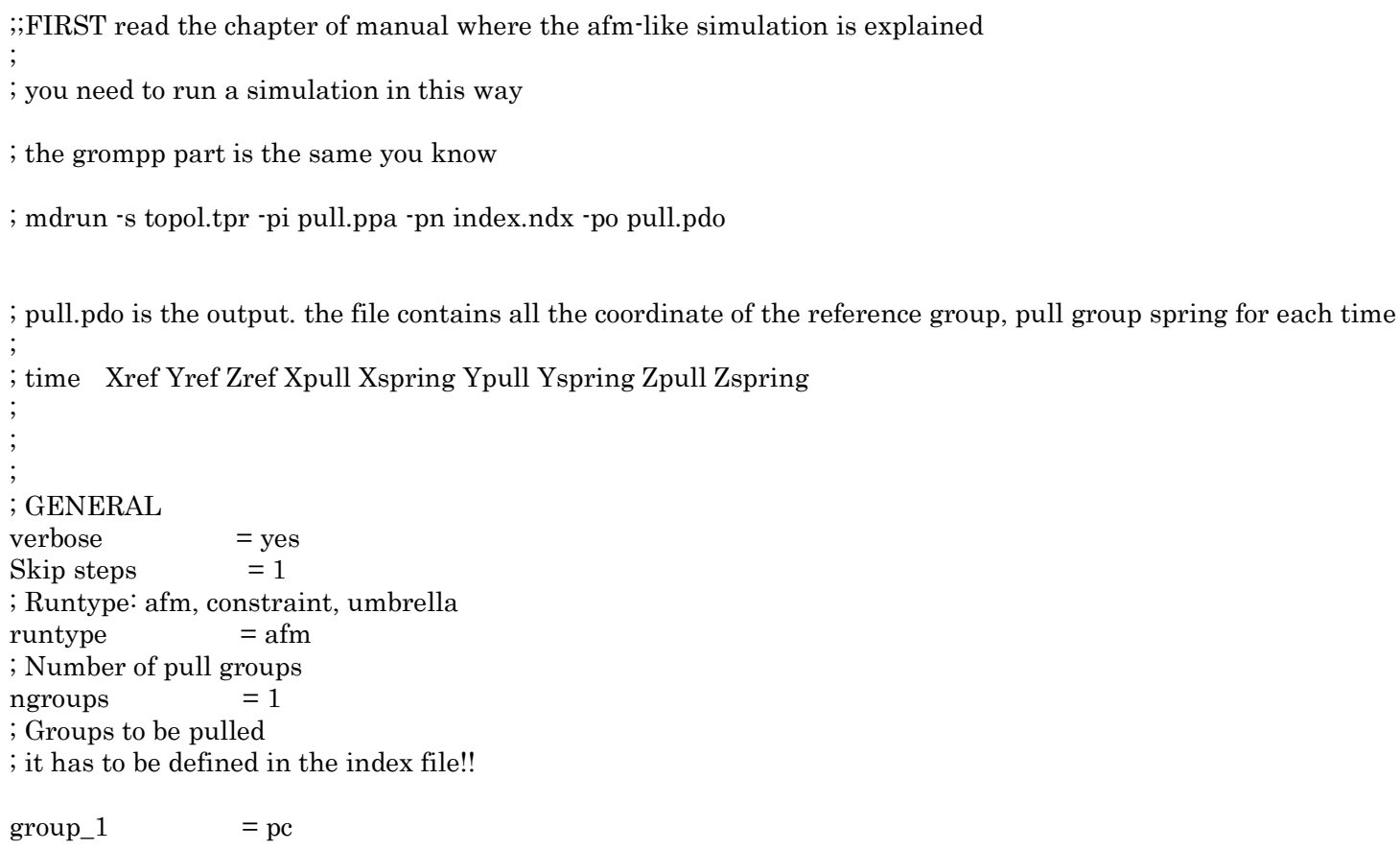




\section{Pulling Simulation $-1.5 \mathrm{~nm}$ spacing Pulling output data file sample}

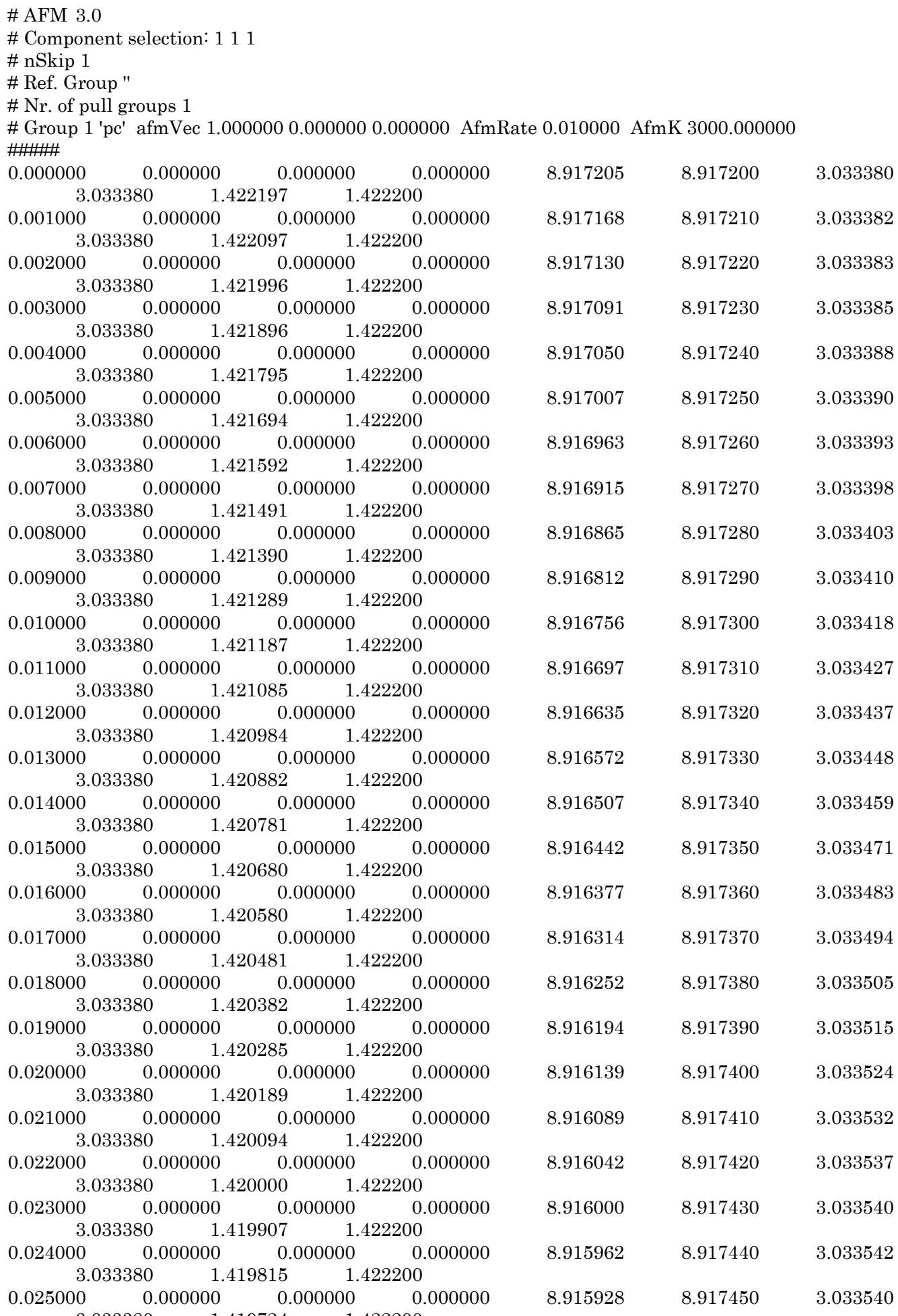




\title{
Force Field Files Atom mass and descriptions (ffG53a6.atp)
}

\author{
O 15.99940 ; carbonyl oxygen $(\mathrm{C}=\mathrm{O})$ \\ OM 15.99940 ; carboxyl oxygen (CO-) \\ OA 15.99940; hydroxyl, sugar or ester oxygen \\ OE 15.99940; ether or ester oxygen \\ OW 15.99940; water oxygen \\ $\mathrm{N} \mathrm{14.00670;} \mathrm{peptide} \mathrm{nitrogen}(\mathrm{N}$ or $\mathrm{NH})$ \\ NT 14.00670 ; terminal nitrogen (NH2) \\ NL 14.00670 ; terminal nitrogen (NH3) \\ NR 14.00670; aromatic nitrogen \\ NZ 14.00670; $\quad$ Arg NH (NH2) \\ NE $14.00670 ; \quad \operatorname{Arg} \mathrm{NE}(\mathrm{NH})$ \\ C 12.01100 ; bare carbon \\ CH0 12.0110 ; bare sp3 carbon, 4 bound heavy atoms \\ CH1 13.01900; aliphatic or sugar CH-group \\ CH 13.01900; aliphatic or sugar $\mathrm{CH}$-group \\ $\mathrm{CH} 214.02700$; aliphatic or sugar $\mathrm{CH} 2$-group \\ CH3 15.03500; aliphatic CH3-group \\ CH4 16.04300; methane \\ CH2R 14.02700 ; $\quad$ CH2-group in a ring \\ CR1 13.01900; aromatic CH-group \\ HC 1.00800 ; hydrogen bound to carbon \\ H 1.00800; hydrogen not bound to carbon \\ DUM 0.00000 ; dummy atom, no idea what the mass should be. PT3-99 \\ S 32.06000 ; sulfur \\ CU1+ 63.54600; copper (charge 1+) \\ CU2+ 63.54600; copper (charge 2+) \\ FE 55.84700; iron (heme) \\ ZN2+ 65.37000; $\quad$ zinc (charge 2+) \\ MG2+ 24.30500; magnesium (charge 2+) \\ CA2+ 40.08000; calcium (charge 2+) \\ P 30.97380; phosphor \\ AR 39.94800; argon \\ F 18.99840; fluor (non-ionic) \\ CL 35.45300 ; chlorine (non-ionic) \\ BR 79.90400; bromine (non-ionic) \\ CMET 15.035 ; CH3-group in methanol (solvent) \\ OMET 15.9994 ; oxygen in methanol (solvent) \\ NA+ $22.9898 ;$ sodium (charge $1+$ ) \\ CL- 35.45300 ; chlorine (charge 1-) \\ CCHL 12.011 ; carbon in chloroform (solvent) \\ CLCHL 35.453 ; chloride in chloroform (solvent) \\ HCHL 1.008 ; hydrogen in chloroform (solvent) \\ SDMSO 32.06000; DMSO Sulphur (solvent) \\ CDMSO 15.03500; DMSO Carbon (solvent) \\ ODMSO 15.99940; DMSO Oxygen (solvent) \\ CCL4 12.011; carbon in carbontetrachloride (solvent) \\ CLCL4 35.453 ; chloride in carbontetrachloride (solvent) \\ FTFE 18.99840; fluor in trifluoroethanol \\ CTFE 12.01100; carbon in trifluoroethanol \\ CHTFE 14.0270 ; $\mathrm{CH} 2$-group in trifluoroethanol \\ OTFE 15.99940 ; oxygen in trifluoroethanol \\ CUREA 12.01100 ; carbon in urea \\ OUREA 15.99940 ; oxygen in urea \\ NUREA 14.00670 ; nitrogen in urea \\ SI $28.08 ;$ silicon \\ MNH3 $0 \quad$; Dummy mass in rigid tetraedrical NH3 group \\ MW $0 \quad ; \quad$ Dummy mass in rigid tyrosine rings
}




\section{Force Field Files Bonded interaction parameter file sample (ffG53a6bon.itp)}

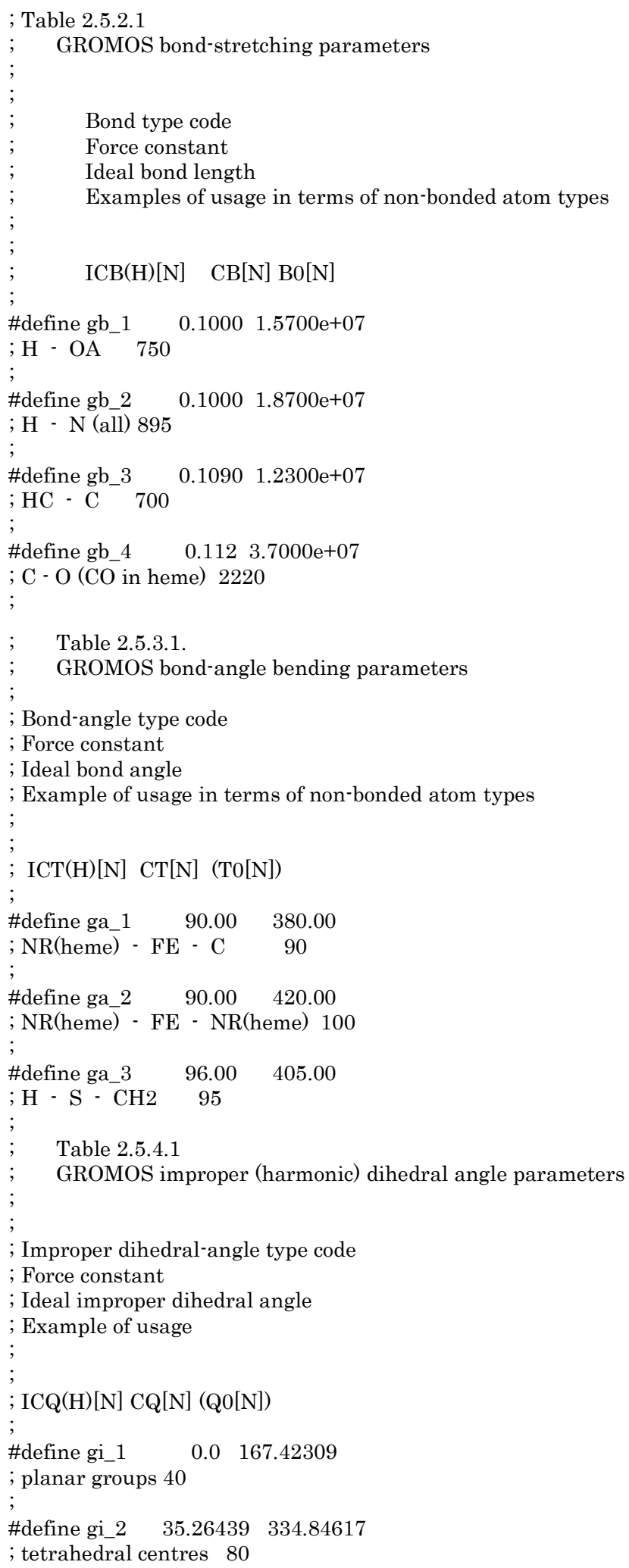




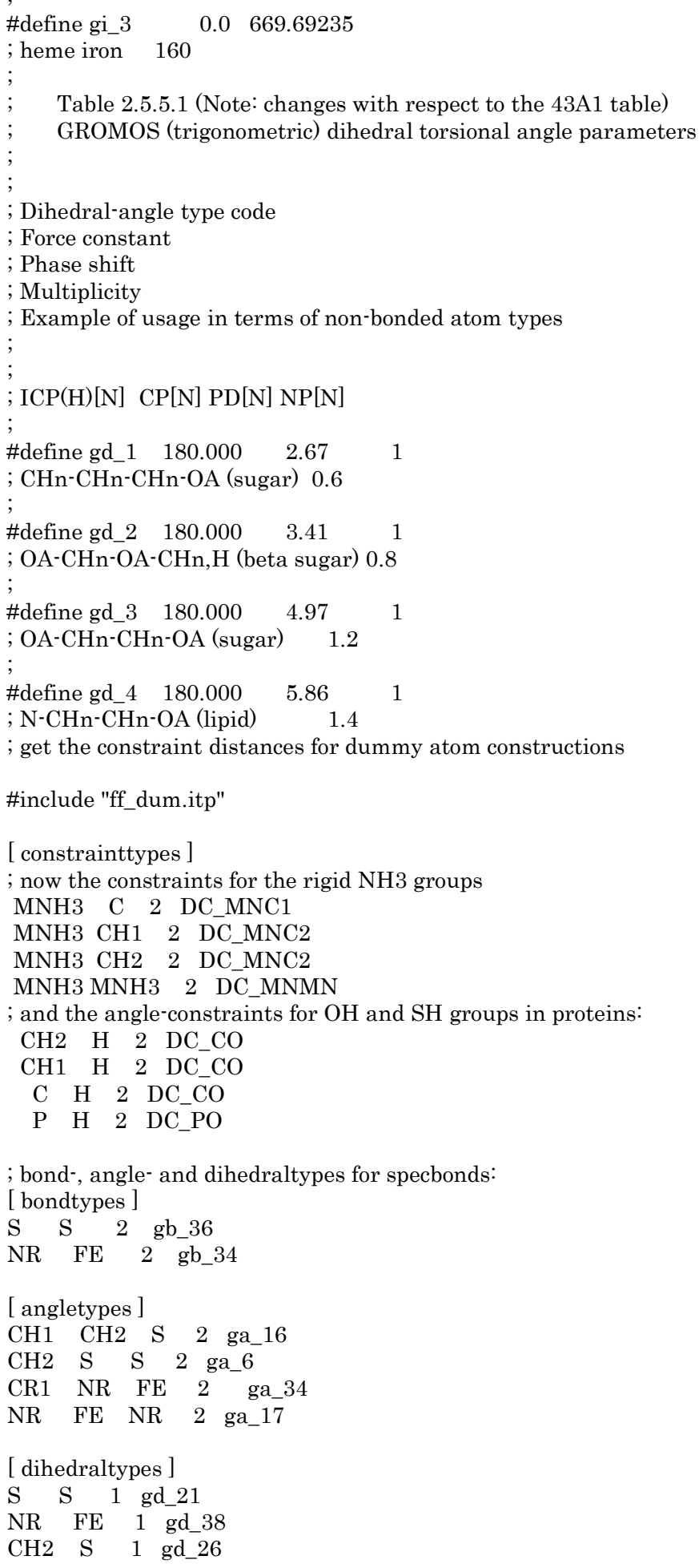

; bond-, angle- and dihedraltypes for specbonds:

[ bondtypes ]

$\mathrm{S} \quad \mathrm{S} 2$ gb_36

NR FE 2 gb_34

[ angletypes ]

$\mathrm{CH} 1 \mathrm{CH} 2 \quad \mathrm{~S} \quad 2$ ga_16

CH2 S S 2 ga_6

CR1 NR FE 2 ga_34

NR FE NR 2 ga_17

[ dihedraltypes ]

$\mathrm{S} \quad \mathrm{S} \quad 1$ gd_21

NR FE 1 gd_38

CH2 S 1 gd_26 


\title{
Force Field Files Non-bonded interaction parameter file sample (ffG53a6nb.itp)
}

\author{
[ atomtypes ] \\ ;name at.num mass charge ptype c6 c12

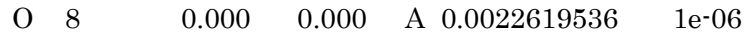 \\ $\begin{array}{llllllll}\text { OM } & 8 & 0.000 & 0.000 & \text { A } & 0.0022619536 & 7.4149321 \mathrm{e}-07\end{array}$

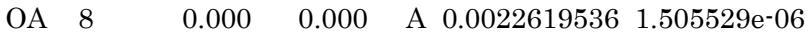

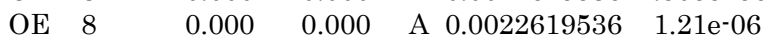 \\ $\begin{array}{lllllll}\text { OW } & 8 & 0.000 & 0.000 & \text { A } & 0.0026173456 & 2.634129 \mathrm{e}-06\end{array}$

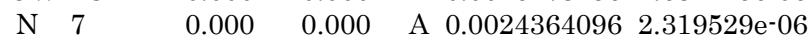

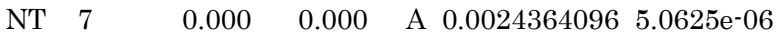

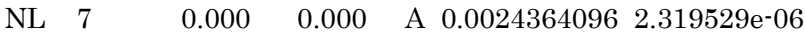 \\ $\begin{array}{llllll}\text { NR } & 7 & 0.000 & 0.000 & \text { A } 0.0024364096 & 3.389281 \mathrm{e}-06\end{array}$

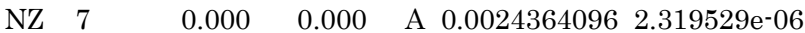 \\ [ nonbond_params ] \\ ; i j func c6 c12 \\ OM $\quad \begin{array}{llllll}O & 1 & 0.0022619536 & 8.611 \mathrm{e}^{-07}\end{array}$ \\ OA $\quad$ O $110.0022619536 \quad 1.38651 \mathrm{e}-06$ \\ OA $\quad$ OM $1 \quad 0.0022619536 \quad 2.258907 \mathrm{e}-06$ \\ $\begin{array}{llllll}\mathrm{OE} & \mathrm{O} & 1 & 0.0022619536 & 1.1 \mathrm{e}^{-06}\end{array}$ \\ OE $\quad$ OM $10.0022619536 \quad 9.4721 \mathrm{e}^{-07}$ \\ OE $\quad$ OA $10.0022619536 \quad 1.505529 \mathrm{e}-06$ \\ [ pairtypes ] \\ ; i j func c6 c12 \\ O $\quad \begin{array}{llllll} & \text { O } & 1 & 0.0022619536 & 7.4149321 \mathrm{e}^{-07}\end{array}$ \\ OM $\quad$ O $110.0022619536 \quad 7.4149321 \mathrm{e}^{-07}$ \\ OM $\quad$ OM $1 \quad 0.0022619536 \quad 7.4149321 \mathrm{e}^{-07}$ \\ OA $\quad$ O $10.0022619536 \quad 9.687375 \mathrm{e}^{-07}$ \\ OA $\quad$ OM $10.0022619536 \quad 9.687375 \mathrm{e}^{-07}$ \\ OA $\quad$ OA $10.0022619536 \quad 1.265625 \mathrm{e}-06$ \\ $\begin{array}{llllllll}\mathrm{OE} & \mathrm{O} & 1 & 0.0022619536 & 9.687375 \mathrm{e}-07\end{array}$ \\ OE $\quad$ OM $1 \quad 0.0022619536 \quad 9.687375 \mathrm{e}-07$ \\ OE $\quad$ OA $10.0022619536 \quad 1.265625 \mathrm{e}^{-06}$ \\ OE $\quad$ OE $10.0022619536 \quad 1.265625 \mathrm{e}^{-06}$ \\ OW $\quad \begin{array}{lllll}\text { O } & 1 & 0.0024331696 & 1.3975653 \mathrm{e}-06\end{array}$ \\ OW $\quad$ OM $10.0024331696 \quad 1.3975653 \mathrm{e}-06$
}

